\title{
Impacts of new and emerging assistive technologies for ageing and disabled housing
}

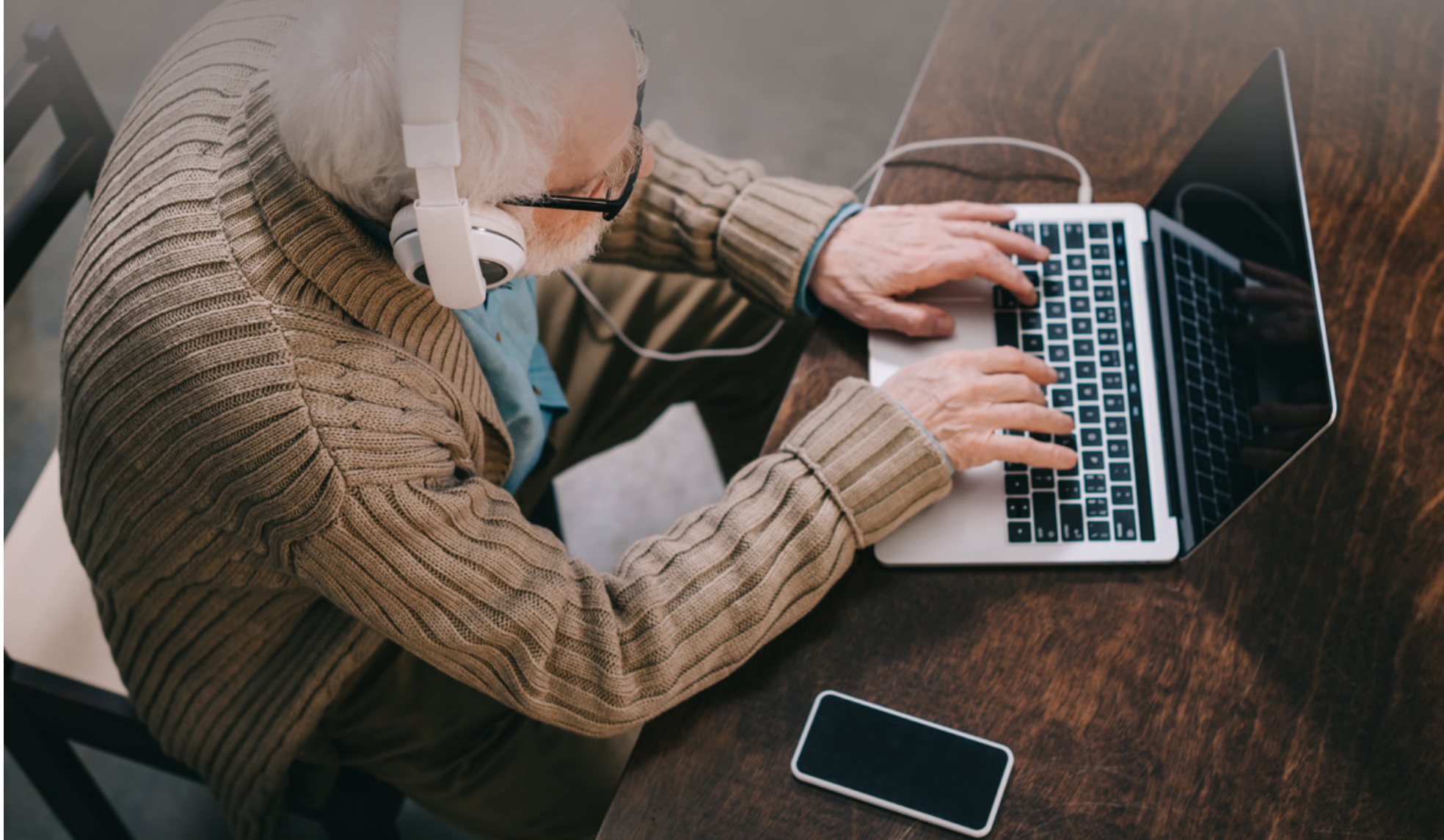

Authored by

Catherine Bridge, The University of New South Wales Fredrick Zmudzki, The University of New South Wales Tracy Huang, The University of New South Wales

Ceridwen Owen, The University of Tasmania

Debbie Faulkner, The University of South Australia
Publication Date December 2021 DOI 10.18408/ahuri7122501

\section{AHURi}


Title

Impacts of new and emerging assistive technologies for ageing and disabled housing

\section{Authors}

Catherine Bridge, The University of New South Wales Fredrick Zmudzki, The University of New South Wales Tracy Huang, The University of New South Wales

Ceridwen Owen, The University of Tasmania

Debbie Faulkner, The University of South Australia

\section{ISBN}

978-1-922498-39-7

\section{Key words}

Ageing in place, community housing, social housing, assistive technology, housing assistance and social policy, housing and the economy, supply of and demand for social and affordable housing.

\section{Series}

AHURI Final Report

\section{Number}

372

\section{ISSN}

1834-7223

\section{Publisher}

Australian Housing and Urban Research Institute Limited Melbourne, Australia

DOI

10.18408/ahuri7122501

\section{Format}

PDF, online only

\section{URL}

https://www.ahuri.edu.au/research/final-reports/372

\section{Recommended citation}

Bridge, C., Zmudzki, F., Huang, T., Owen, C. and Faulkner, D. (2021) Impacts of new and emerging assistive technologies for ageing and disabled housing, AHURI Final Report No. 372, Australian Housing and Urban Research Institute Limited, Melbourne, https://www.ahuri.edu.au/research/ final-reports/372, doi: 10.18408/ahuri7122501.

\section{AHURI}

AHURI is a national independent research network with an expert not-for-profit research management company, AHURI Limited, at its centre.

AHURI's mission is to deliver high quality research that influences policy development and practice change to improve the housing and urban environments of all Australians.

Using high quality, independent evidence and through active, managed engagement, AHURI works to inform the policies and practices of governments and the housing and urban development industries and stimulate debate in the broader Australian community.

AHURI undertakes evidence-based policy development on a range of priority policy topics that are of interest to our audience groups, including housing and labour markets, urban growth and renewal, planning and infrastructure development, housing supply and affordability, homelessness, economic productivity, and social cohesion and wellbeing.

\section{Acknowledgements}

This material was produced with funding from the Australian Government and state and territory governments. AHURI Limited gratefully acknowledges the financial and other support it has received from these governments, without which this work would not have been possible.

AHURI Limited also gratefully acknowledges the contributions, both financial and in-kind, of its university research partners who have helped make the completion of this material possible.

\section{Disclaimer}

The opinions in this report reflect the views of the authors and do not necessarily reflect those of AHURI Limited, its Board, its funding organisations or Inquiry Panel members. No responsibility is accepted by AHURI Limited, its Board or funders for the accuracy or omission of any statement, opinion, advice or information in this publication.

\section{AHURI journal}

AHURI Final Report journal series is a refereed series presenting the results of original research to a diverse readership of policymakers, researchers and practitioners.

\section{Peer review statement}

An objective assessment of reports published in the AHURI journal series by carefully selected experts in the field ensures that material published is of the highest quality. The AHURI journal series employs a double-blind peer review of the full report, where anonymity is strictly observed between authors and referees.

\section{Copyright}

(C) Australian Housing and Urban Research Institute Limited 2021

This work is licensed under a Creative Commons Attribution-NonCommercial 4.0 International License, see http://creativecommons.org/licenses/by-nc/4.0/. 


\section{Contents}

List of tables

List of figures vi

Acronyms and abbreviations used in this report

Executive summary $\quad 1$

1. Introduction $\quad 4$

1.1 Introduction $\quad 5$

1.2 Definition of assistive technology $\quad 5$

$\begin{array}{ll}1.3 \text { What is a smart home? } & 6\end{array}$

1.4 Support systems - aged care and disability policy and AT

1.4.1 National Disability Insurance Scheme

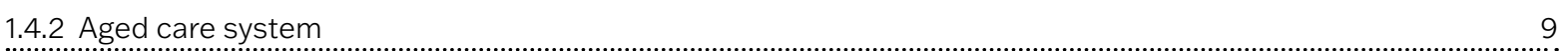

1.5 Existing research

1.6 The study 12

1.7 Research approach $\quad 12$

1.7.1 Qualitative analysis $\quad 12$

1.7.2 Economic modelling $\quad 13$

$\begin{array}{lr}\text { 1.7.3 Limitations } & 15\end{array}$

2. Smart home assistive technology 17

2.1 A call for international framework for AT provisions 21

2.2 Current trends in smart homes $\quad 22$

2.2.1 Device and service market $\quad 22$

2.2.2 Reliance on periphery services

$\begin{array}{lr}2.3 \text { Smart home as AT } & 23\end{array}$

2.3.1 What are the benefits of a smart home?

2.3.2 Effectiveness of smart home AT 25

2.3.3 Dwelling design considerations

2.3.4 What are the risks of smart home AT?

$\begin{array}{ll}\text { 2.3.5 The smart home AT market } & 27\end{array}$

2.3.6 Smart home AT costs

$\begin{array}{ll}2.4 \text { Policy development implications } & 28\end{array}$

3. User survey findings $\quad 30$

3.1 Survey development 31

3.2 Survey methods and sampling $\quad 33$

3.3 User survey findings $\quad 34$

3.3.1 Survey demographics $\quad 34$

3.3.2 Understanding respondent's residential accommodation $\quad 36$

3.3.3 AT funding sources

3.3.4 AT used by respondents $\quad 38$ 
3.4.1 Smart home AT product costs

3.4.2 Housing provider and commercial smart home segment

\subsection{Smart home benefits}

3.5.1 Improved quality-of-life

3.5.2 Improved quality-of-life and extended independent life

3.5.3 Smart home efficiency

3.5.4 Economics of improved safety outcomes

3.5.5 Economics of improved physical activity

3.5.6 Economics of mental health outcomes

3.5.7 Economics of healthcare monitoring

3.6 Economic modelling

3.6.1 Health-related quality-of-life

\subsection{Effectiveness and cost-effectiveness}

3.7.1 Smart home product effectiveness

4. Service provider and individual user views on Assistive Technology

4.1.1 Perceived usefulness

4.1.2 Perceived ease of use

\subsection{Housing and service providers}

4.2.1 Infrastructure

4.2.2 Knowledge and innovation

4.2.3 Interface between housing and care $\quad 74$

4.3 Ethics

4.3.1 Care

4.3.2 Data privacy $\quad 76$

4.3.3 Equity of access to AT $\quad 78$

4.4 Policy development implications $\quad 79$

5. Policy development options $\quad 80$

5.1 What are the implications of new and emerging AT for older people and those living with disability? 80

5.1.1 How do new and emerging AT for older people and those living with disability interact with policy and practice? 
Appendix 2: List of organisations contacted for user survey distribution

Appendix 3: NDIS AT complexity table 


\section{List of tables}

Table 1: Assistive technology definitions 6

Table 2: NDIS support categories 9

Table 3: Search terms

Table 4: Examples of smart home technologies

Table 5: What is your age group?

Table 6: Geolocation by state or territory and gender

Table 7: Martial status of respondents 36

Table 8: Primary accommodation of respondents

Table 9: Percentage of respondents by type of residential accommodation

Table 10: Length of residency in current accommodation

Table 11: Descriptive statistics for the QUEST 2.0 from the smart home AT Survey $(n=20)$

Table 12: Item by item analysis for the Device sub-scale for smart home AT in the home $(n=21)$

Table 13: Indicative smart home provider requirements specification 49

Table 14: AQoL 4D overall utility scores before and after AT - by cost category 55

Table A1: New South Wales focus group one attendees 109

Table A2: New South Wales focus group two attendees

Table A3: South Australia focus group attendees $\quad 112$

Table A4: Online Zoom focus group one attendees 114

Table A5: Online Zoom focus group two attendees

Table A6: Summary list of all attendees at all focus groups 116

Table A7: NDIS SDA guidelines appendix A mention of automation or smart homes 117 


\section{List of figures}

Figure 1: Smart home ecosystem diagram

Figure 2: Economic data components for AT products

Figure 3: Respondents who identified one or more functional impairments

Figure 4: Percentage of respondents by the type of smart home AT in use

Figure 5: Rooms in the home where that the smart home AT system is deployed $\quad 40$

Figure 6: Overall satisfaction of AT by category

Figure 7: Most satisfied assistive technology

Figure 8: Change in Activities of Daily Living (ADL) status using smart home AT (eg. bathing, dressing, etc.) 43

Figure 9: Change in Intrinsic Activities of Daily Living (IADL) status using smart home AT (e.g. cooking, house cleaning, etc.)

Figure 10: Change in perceived quality of relationships

Figure 11: Change in perceptions of social isolation

Figure 12: Perceived change with visual activities

Figure 13: Perceived change with auditory activities

Figure 14: Perceived change with communication abilities

Figure 15: Perceived change with sleep quality

Figure 16: Perceived change with pain experienced

Figure 17: Total upfront cost of assistive devices with and without government support $\quad 48$

Figure 18: Out of pocket cost of assistive devices with and without government support 48

Figure 19: Ongoing annual cost of assistive device with and without government support

Figure 20: AQoL-4D standardised mean scores before and after AT installation $\quad 51$

Figure 21: AQoL-4D standardised mean scores before and after AT - by cost category 51

Figure 22: Indicative assistive technology costs and effectiveness by cost category

Figure 23: Estimated incremental cost-effectiveness - low-cost scenario

Figure 24: Estimated incremental cost-effectiveness - NDIS scenario

Figure 25: Photo of Eric's DIY set-up that needs replacing with new smart home technologies

Figure 26: Example of smart home technology

Figure 27: Example of smart watch control interface and health monitoring $\quad 67$

Figure 28: Photo of Mark's Google Home set-up 


\section{Acronyms and abbreviations used in this report}

AAATE Association for the Advancement of Assistive Technology in Europe

AHURI Australian Housing and Urban Research Institute Limited

ABS Australian Bureau of Statistics

ACAT Aged Care Assessment Team

ACQS Royal Commission into Aged Care Quality and Safety

ADL Activities of Daily Living

AHURI Australian Housing and Urban Research Institute Limited

Al Artificial Intelligence

AlHW Australian Institute of Health and Welfare

ANZ/ISO International Standards adopted by Australia and New Zealand

AQoL Assessment of Quality-of-life

AQoL-4D Assessment of Quality-of-life 4 Dimensions

AT Assistive Technology

ATSE Academy of Technological Sciences and Engineering

CALD Culturally and Linguistically Diverse

CHSP Commonwealth Home Supported Program

CRPD United Nations Conventions on the Rights of Persons with Disabilities

CUA Cost Utility Analysis

DSS Department of Social Sciences

$\mathrm{EACH}$ Extended Aged Care at Home

EACH-D Extended Aged Care at Home - Dementia

EASTIN European Assistive Technology Information Portal

EAT Electronic Assistive Technology

ECU Environmental Control Unit

FND Functional Neurological Disorder

GATE Global Cooperation on Assistive Technology

GDPR General Data Protection Regulation

GPS Global Positioning System

GREAT Global Research, innovation and Education in Assistive Technology

GST Goods and Services Tax

HTA Health Technology Assessment

IADL Intrinsic Activities of Daily Living

ICF International Classification of Functioning, Disability and Health

ICT Information and Communication Technology

IoT Internet of Things

LAC Local Area Coordinator

NBN National Broadband Network

NCC National Construction Code 


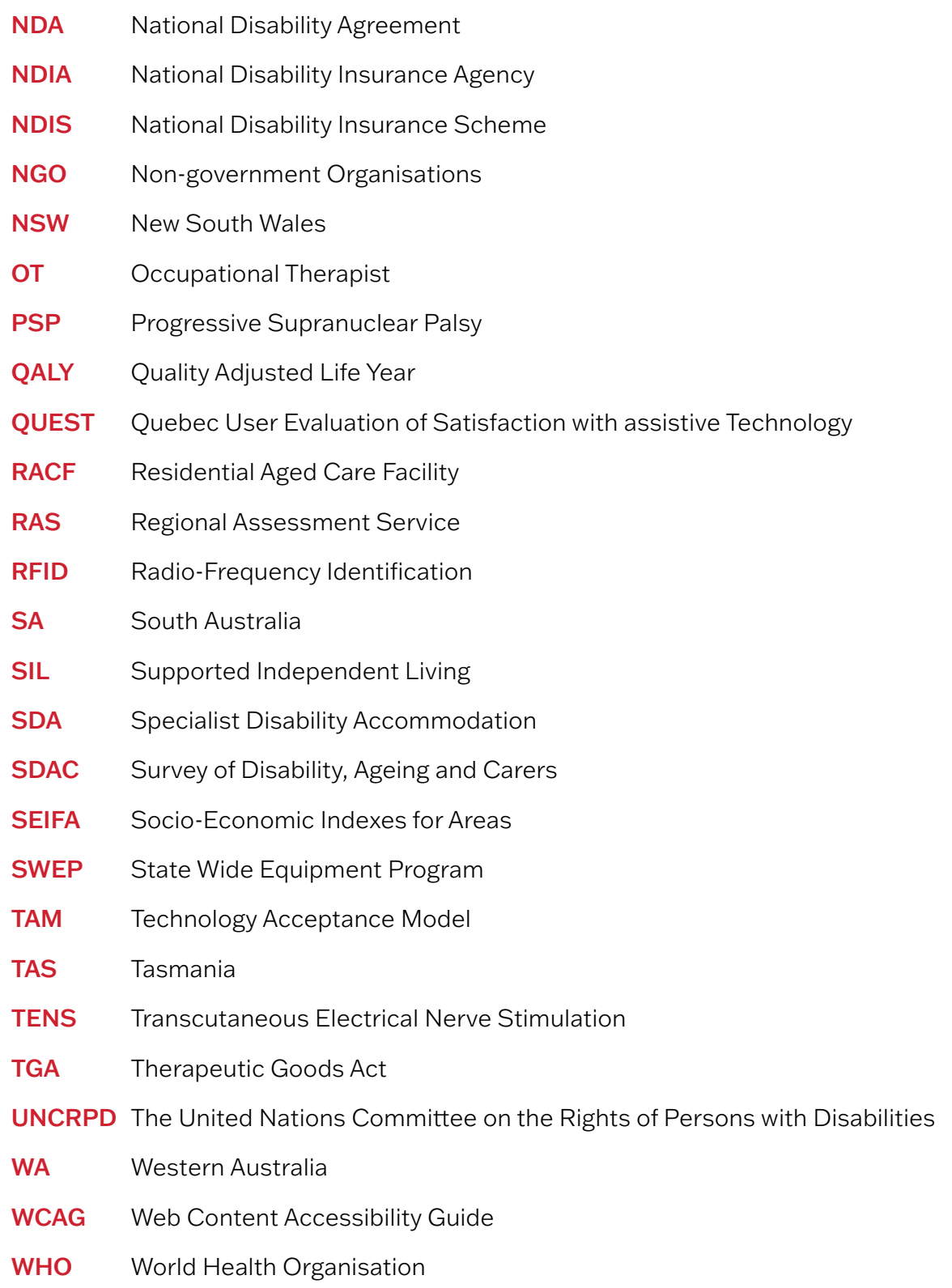




\section{Executive summary}

\section{Key points}

Key findings include the following:

- Smart home Assistive Technology (AT) has the potential to improve quality-of-life, reduce reliance on care and reduce the cost of care for those with functional limitations associated with ageing or the onset of disability.

- Smart home AT may potentially indirectly support a range of ongoing benefits, including extended independent living, smart home energy efficiency, safety and security, physical and mental activity and healthcare monitoring.

- Most end-users reported overall high satisfaction with their smart home AT. Satisfaction was higher for assistive technology devices, and lower for services.

- Average smart home AT costs over five years were approximately $\$ 700$ to $\$ 800$, with variations ranging from greater than $\$ 1,000$ to below $\$ 600$. This is an emerging market with considerable variation in product ranges, specifications, functionality, quality and cost.

- An information gap exists, characterised by lack of clarity about the role of funders, housing designers, housing providers and for individuals with functional limitations in their knowledge and skills concerning what can be done and what is safe, sustainable and effective.

- Lack of coordination, regulation, guidance and the absence of an evidence-base about what works for who, in what context, exacerbates the existing apathy and confusion around smart home funding guidance. 


\section{- Commercial aged care and disability housing providers reported uptake of smart home AT policies where a market advantage was perceived, whilst social housing agencies found smart home AT out of scope and unaffordable.}

This research sought to explore the potential for smart AT to reduce the need for support services, and to measure in economic terms if so, how, and to what extent. Smart home AT can be defined as 'any device that helps a person with a disability achieve a more independent and productive life ... using generic smart home technology' (Pennsylvania Assistive Technology Foundation (PATF) 2021).

\section{Key findings}

Smart home AT has the potential to improve quality-of-life, reduce the reliance on care and facilitate autonomy for those with limitations of function. The research indicates smart home technologies support improved independence, activity, social and community connectedness and, as a result, improved physical and mental health outcomes. Quality-of-life measures indicated improvements consistently across all dimensions, including independent living, mental health, relationships, and senses.

Early adopters of smart home AT are predominantly self-funders and whilst there is high variation and limited data there is evidence of potential cost-efficiencies. With smart home AT being an emerging segment of AT there is a high variation in product ranges, specifications, functionality, quality and cost. Smart home interventions cross over multiple technologies that are dynamic and developing quickly. The actual devices have a physical focus, but the ubiquitous expansion of cloud-based platforms underpins low-cost integration of applications and devices and novel flexibility in the building of virtual smart home environments. The value of smart home AT is implicitly driven by the ability to customise the technology and design systems that meet individual goals and needs. Despite high variation in smart home AT costs between respondents, the data was indicative of cost-effectiveness in terms of reduced care. Whilst the sample size was limited, our results established that the average smart home AT set-up costs per household over a five-year period were around $\$ 700$ to $\$ 800$, with the variation ranging from above $\$ 1,000$ to below $\$ 600$.

This project identified a sustained gap in piloting and development of smart home AT government policy, previously recommended through initial Australian research in 2012. This has resulted in a limited consumer base for economic evaluation of smart home AT and a corresponding gap in published literature. The lack of clear policy frameworks and insufficient coordination has resulted in an ad-hoc and piecemeal implementation practice with many who could potentially benefit not having the skill, knowledge or financial ability to invest. In this context, the frameworks to promote the deployment of smart home AT are lacking or are confusing. There is a critical lack of clarity about the role of funders, housing designers, housing providers and individuals associated with smart home AT.

Existing AT policy is vague about deliverables, and typically mainstream services and supports (e.g., smartphones, tablets and 'apps') are considered as being outside of the scope of government funding. Currently, there is no strategy for predicting how more innovative and sophisticated devices and software developed for the disability sector will emerge or be integrated into housing. A plethora of technologies and rapid technological advancements present challenges for both policymakers and clients in the decision-making process, including identifying and evaluating appropriate technologies, and providing flexible funding schemes that support customisation and ease of procurement. AT, housing design and care need to be considered holistically. This presents enormous opportunities for innovation that can better support a better quality-of-life outside the more traditional institutional and group accommodation models. 


\section{Policy development options}

The potential economic and social benefits of policies and practices within Australia for smart home AT are unclear. A better understanding of these benefits will facilitate an evidence-base for the development, adoption and uptake of smart home AT that is useful and cost-efficient. The development of policy in smart home AT is important; it will provide an overarching vision and outline resourcing priorities. The digital revolution is already well underway, and the planning for, and investment in, evidence-based guidance should be a key policy initiative. Current policies must be reviewed in relation to smart home AT for older people and those living with a disability if Australia is to increase uptake and leverage economic and cost benefits, whilst improving outcomes. This is especially important in avoiding the risk of a wider digital and technological divide for those with less disposable income in social housing and rental accommodation.

Across both the disability and aged care sectors, there is the need to increase transparency for practitioners and end-users on eligibility and availability of AT interventions. Importantly, policy needs to establish and support ongoing education to facilitate competency development in this area for housing practitioners, AT suppliers and NDIS and Regional Assessment Service (RAS) and Aged Care Assessment Team (ACAT) assessors. Pooling resources through innovative financing, such as co-financing, should be investigated. This may decrease the existing fragmentation of available resources, whilst facilitating demand.

On the supply-side, if practitioners were willing to share their knowledge and preferred products, this would increase market transparency and collaboration, and may strengthen procurement for context-appropriate smart home AT whilst decreasing the fragmentation. The lack of collaboration and guidance are major barriers to fostering a climate of innovation and efficiency that can maximise the gains from this AT.

There is also a lack of data to build better evidence-based practice guidelines. The practitioners we interviewed understood that transformational change also meant addressing the market and better understanding the supply and demand-side barriers such as costs and the issue of who pays. Nevertheless, the adoption of smart home AT needs to be supported by broad consultation designed to maximise existing knowledge, increase awareness, strengthen systems, and improve service delivery outcomes. Accomplishing this requires a cross-sector partnership acting as a catalyst for change. Importantly, practitioners need to actively generate policy proposals that are technically, economically, and politically feasible, grounded within the language and value system practised by the policymakers. A national round table discussion on the way forward may be a good step in this direction.

Further, better articulation of smart home AT product profiles targeted for defined eligibility may improve the most efficient targeting of funding and servicing whilst maximising smart home AT net benefit into the future. This could include an independent national education model and a specialist advisory group that can bring together and coordinate the necessary agencies responsible for policy and practice.

\section{The study}

This research was undertaken in the context of both aged care and disability reforms, aimed at improving consumer choice whilst maximising safety and independence in housing or accommodation that is fit for purpose and that can maximise or compensate for losses in functional capacity. This research was guided by the following questions:

- What are the current and emerging directions in the development of AT globally and nationally?

- What are the current policy directions in disability and aged care in Australia and internationally for smart home AT?

- What factors enable or constrain the use of smart home AT to support independent living in practice?

- What are the potential economic and social benefits of policies and practices to support the development and adoption of smart home AT in Australia? 


\section{Introduction}

- The proportion of older Australians and those with disabilities is increasing, as is the demand for services to enable people to live as independently as possible.

- The desire of older people and people with disability to live independently means an increasing demand for services will not only require substantially more funding but also innovative new approaches to delivering effective and cost-effective care in a home environment.

- AT policy has traditionally not considered the implications for residential housing outside of home modification practice.

- An important mainstream technology for people living with disabilities and older people is the concept of the 'smart home'. This has the ability, when linked to AT, to help people achieve a more independent and productive life.

- Smart home AT is currently not part of the policy discussion in aged care or disability services.

- Empirically, the report draws on a survey of 54 respondents, five focus groups with housing and AT providers and Interested Non-Government organisations, and four in-depth case studies.

- The survey provided the basis for the economic analysis and recruitment for the case studies, which was voluntary.

- The focus groups were transcribed and then thematically analysed to explore the participants' experiences and views about the factors that were impacting the rollout, purchase and maintenance of smart home technology in residential accommodation. 


\subsection{Introduction}

The proportion of older Australians and those with disabilities is increasing, as is the demand for services to enable people to live as independently as possible. The clear preference for independent living arrangements (Productivity Commission 2015) and flexible choices for aged care and disability support services, including home modifications, are reflected in policy planning and the broader reform agenda for both the NDIS and the Commonwealth Aged Care Reform (Productivity Commission 2011a; Productivity Commission 2011b).

Based on 2016 Census data, the proportion of people aged 65 years or over is projected to increase from 15 per cent on 30 June 2017 to between 21 and 23 per cent in 2066 (ABS 2018a). As the population ages, an increasing number of people are living with chronic health conditions and limitations on their ability to undertake daily tasks (Australian Institute for Health and Welfare 2019).

In 2018, the Australian Census revealed that there were 4.4 million Australians with disability, representing 17.7 per cent of the population, with prevalence increasing with age from one in nine (12\%) for people aged 0-64 years to one in two (50\%) for people aged 65 years and over (ABS 2018a). Of the 4.4 million Australians with a disability, over half (53\% or 2.3 million) used aids or equipment because of their condition, increasing to 58 per cent of those who live alone.

The desire of older people and people with disability to live independently means an increasing demand for services will not only require substantially increased funding but also innovative new approaches to delivering effective and cost-effective care in a home environment. In this regard, it is increasingly well recognised that more flexible support options have a role in reducing pressures on the aged care, disability and healthcare systems. In this context, people with disability represent a substantial population that may benefit from the integration of a greater range of AT products to improve their independence and increase their participation in social and economic life. Internationally and nationally, AT consumer products and health technologies are viewed as essential technologies for ameliorating functional impairment, enabling ageing in place, and improving quality-of-life. For example, the World Health Organisation (2015) views access to appropriate AT as indispensable to health, productivity and ageing over time. AT has the potential to improve the quality-of-life, mobility and independence of many Australians, enabling them to continue living at home and to remain connected to their communities for longer.

\subsection{Definition of assistive technology}

Traditionally, AT has been designed in a bespoke manner for people with disabilities to address a specific limitation, helping the user complete a task they would otherwise be unable to do. In some cases, the technology that is developed for a targeted group of people is then marketed to the wider public as it increases ease of use or convenience for everyone. Unlike traditional AT, the 'smart home' and the Internet of Things (IoT) have been developed for the mainstream market, targeting the general public. However, both have enormous potential as AT for people living with disabilities. The definition of what is then considered and classified as AT within government and funding bodies is critical-it determines what can and cannot be funded.

Globally, the commonly used definition of AT is provided by the World Health Organisation (WHO). WHO defines AT as 'an umbrella term covering the systems and services related to the delivery of assistive products and services' (WHO 2018). The Australian and New Zealand and International Organization for Standards classification of technical aids defines AT as 'any product, instrument, equipment or technical system used by a disabled person, especially produced or generally available, preventing, compensating, monitoring, relieving or neutralising disability' (ISO 2016). In Australia, the National Disability Insurance Scheme (NDIS) uses the term 'assistive technology' as defined by WHO, whilst many jurisdiction-based funders used the terms 'aids and equipment', including the Australian Bureau of Statistics (ABS) national Survey of Disability, Ageing and Carers (SDAC). Table 1 lists a range of definitions provided by government organisations, peak bodies and industry providers. Irrespective of the variances in terminology, it is important to recognise that both definitions provided by the WHO and ANZ/ISO stipulate any product can be an AT if it is used by a person with disability to improve or perform tasks that they would otherwise be unable to do without the technology. In addition, it is irrelevant whether the technology was developed or adapted specifically for people with disabilities or available for mainstream markets. 
Table 1: Assistive technology definitions

\begin{tabular}{ll}
\hline Organisation & Definition \\
\hline $\begin{array}{l}\text { ANZ/ISO 9999: 2016 - Assistive } \\
\text { products for persons with disability } \\
\text { - Classification and terminology }\end{array}$ & $\begin{array}{l}\text { Any product, instrument, equipment or technical system used by a disabled person, } \\
\text { especially produced or generally available, preventing, compensating, monitoring, } \\
\text { relieving or neutralising disability (ISO 2016). }\end{array}$ \\
\hline Assistive Technologies Australia & $\begin{array}{l}\text { Assistive Technology (AT) is any device, system or design used by individuals to } \\
\text { perform tasks that might otherwise be difficult or impossible. AT range from simple } \\
\text { household items like a jar opener to the more complex items like a pressure care } \\
\text { mattress that prevents pressure sores. In short, anything that assists individuals } \\
\text { to perform daily activities can be considered assistive technology (Assistive }\end{array}$ \\
$\begin{array}{ll}\text { Technologies Australia 2020a). } \\
\text { Independent Living Centres }\end{array}$ & $\begin{array}{l}\text { Assistive Technology is any device, system or design that allows an individual to } \\
\text { perform a task that they would otherwise be unable to do, or that increase the ease } \\
\text { and safety with which a task can be performed (Independent Living Centres 2020b). }\end{array}$ \\
\hline NDIS SDA Design Standards & $\begin{array}{l}\text { Any device or system that allows individuals to perform tasks they would otherwise } \\
\text { be unable to do or increases the ease and safety with which tasks can be performed } \\
\text { (NDIS 2019b). }\end{array}$ \\
\hline SDAC ABS & $\begin{array}{l}\text { Aids and equipment refer to 'any devices used by a person with one or more } \\
\text { disabilities to assist them with performing tasks' (ABS 2018b). }\end{array}$ \\
\hline World Health Organisation & $\begin{array}{l}\text { An umbrella term for any device or system that allows individuals to perform tasks } \\
\text { they would otherwise be unable to do or increases the ease and safety with which } \\
\text { tasks can be performed (WHO 2004). }\end{array}$ \\
\hline
\end{tabular}

Source: Collated definitions with each cited source referenced individually in the table.

\subsection{What is a smart home?}

An important mainstream technology for people living with disabilities and older people is the concept of the 'smart home'. Although the idea of an intelligent home that allows users to automate tasks is not new, the advances made in Artificial Intelligence (Al) and blockchain technologies in recent years has enabled the loT and smart devices that bring the smart home concept to life. As technology is now, more than ever, embedded into our everyday lives, from the way we work, communicate, and carry out everyday activities such as cooking and cleaning, the smart home market has continued to grow. As a result, smart home technologies are now more widely available at lower price points with more choices and variety that has significant benefits for older people and those with disabilities.

A smart home integrates technologies and services in the home with the purpose of automating tasks with increasing comfort, safety and security, and energy optimisations (Saizmaa and Kim 2008). The smart home is therefore an ecosystem of independent and interdependent technological devices and programs that communicate with each other through a wired or wireless connection that is able to respond and modify itself continuously to the needs of the user (Bierhoff et al. 2007). For example, a user will be able to pre-program the system to turn off the lights, shut the windows, and switch off all power when they are leaving home, and the system will do so when the user says 'leaving home' or by activating the sequence of activities through a mobile interface. There are many definitions within academic literature of what is a smart home. Alam et al. (2012) provides the most comprehensive definition of a smart home that describes the three key components a home must have to be "smart":

1. Internal networks (wire, cable or wireless) that enable devices to communicate with each other

2. An intelligent control (a way to manage the features in the system

3. Home automation (products and remote access).

These three key components are illustrated in Figure 1. 
For the purpose of this research, a smart home is defined as "an application of ubiquitous computing that is able to provide user context-aware automated or assistive services in the form of ambient intelligence, remote home control, or home automation" (Alam et al. p, 1, 2012). In this definition, the smart home offers user control of home appliances and devices through an interface or voice activated virtual assistant. The ambient intelligence systems built into the devices monitor the home to provide customisations and optimisations that can be tailored to the users' preferences and behaviours (Alam et al. 2012). The ecosystem and relationship between the virtual assistant to devices is also illustrated in Figure 1.

Figure 1: Smart home ecosystem diagram

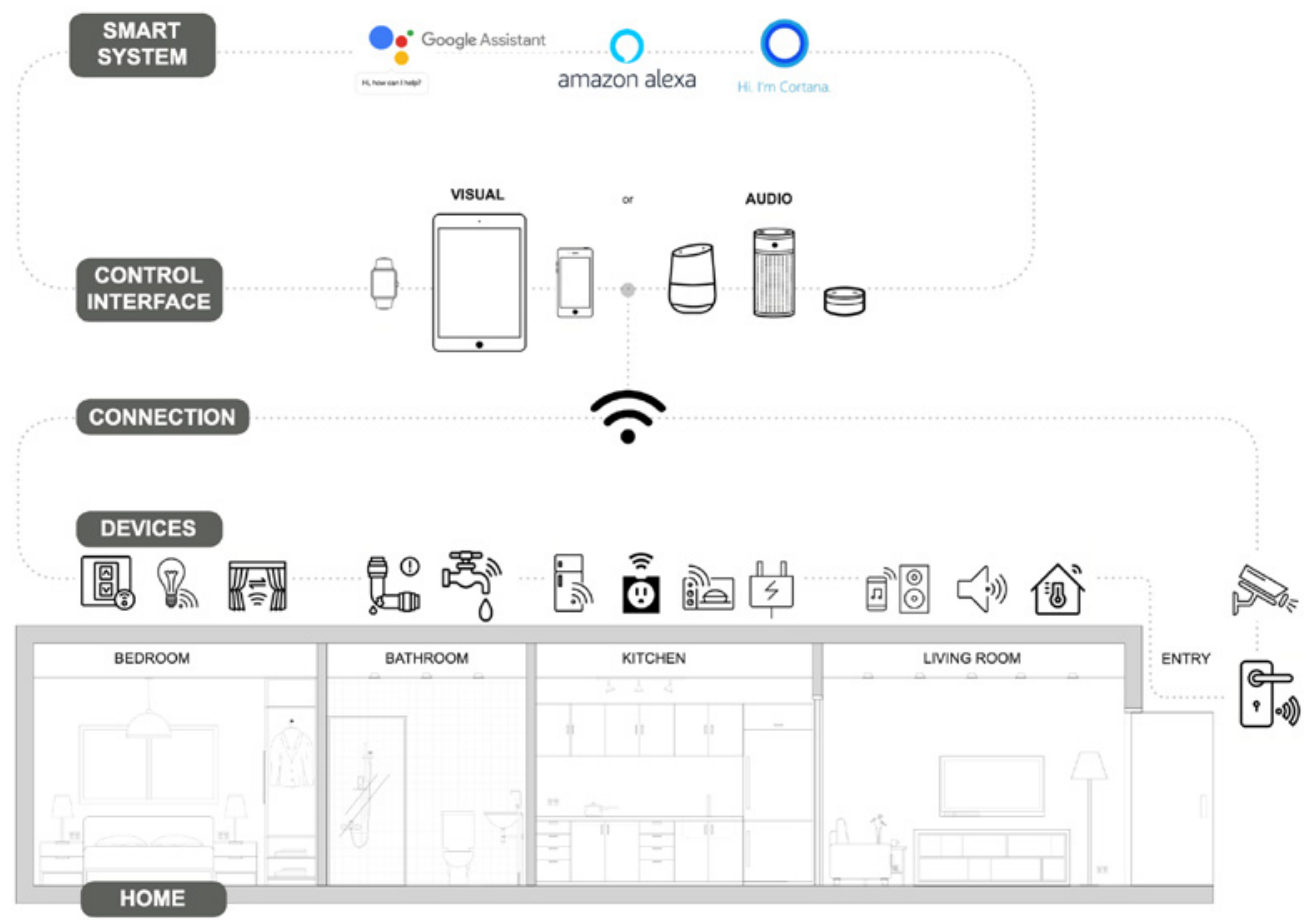

Source: Authors.

As we can see from Figure 1 above and the previous discussion, smart home AT can be defined as 'any device that helps a person with a disability achieve a more independent and satisfying life' (Pennsylvania Assistive Technology Foundation (PATF), 2021) using generic smart home technology.

\subsection{Support systems - aged care and disability policy and AT}

In Australia, if you are over 65 years of age and not part of the National Disability Insurance Scheme (NDIS), you are eligible to apply for funding support for home modifications through the aged care system. If you are under the age of 65 with a disability, you are eligible for support under the NDIS if it is currently available in your respective state or territory.

In 2018, more than 50 per cent of people with disability (2.3 million) used a form of aids or equipment to assist and support their ability to remain in their own homes. In addition, of the 4.2 million people with disability living in households, 12.2 per cent had made some form of home modification (ABS 2018b).

The use of AT increases with age and the severity of disability related restrictions (Bricknell 2003), which is unsurprising if one views these types of interventions as critical to remaining within the community and outside institutional care. Nevertheless, this same research found that an absence of adequate assistance was a continuing problem that impinged on quality-of-life and that there "remained a substantial proportion of people whose needs were not met at all" (Bricknell 2003: 49). 


\subsubsection{National Disability Insurance Scheme}

Although the definition of AT used by the NDIS is inclusive of any mainstream technologies, there is limited advocacy or recognition of smart home technologies in funding guidelines. The NDIS fails to clearly include or differentiate smart home technologies from established AT items, generally physical equipment and home modifications such as ramps or handrails. The NDIS Act Review 2019 emphasises a focus on supporting specific cohorts (including children and people with disability) in procuring assistive technologies and home modifications (Department of Social Sciences 2019). However, the current categorisations and the differing levels of complexity definitions of AT means that navigating the NDIS system is potentially problematic for many. The NDIS catalogue is predominately focused on people and services, with less than 20 per cent of the catalogue relating to AT equipment and less than 1 per cent to home modifications (National Disability Insurance Agency 2019). Additionally, the categories are not exclusive and not mutually inclusive, presenting challenges where smart home AT fall under multiple categories and levels of complexity. Examples of this are provided in Appendix 3: NDIS AT complexity table, where smart home equipment and service required overlap across many catalogues and complexity levels.

The NDIS aims to give choice and control to participants in a self-directed care model that has meant a shift of responsibility from the state to the individual. The inherent shift away from standardised services raises questions about the balance between choice and control, and safeguards for vulnerable individuals (Olney and Dickinson 2019). The lack of any explicit reference to smart home AT leaves NDIS participants particularly vulnerable whether they are self-managed, plan managed, NIDA managed or a combination of all. Self-managed participants administering their own plans have to undertake independent research on any new and emerging technologies and advocate for what is 'reasonable and necessary', exacerbating the existing administrative pressures to navigate the NDIS bureaucratic process (Malbon and Carey 2019). Participants who are NDIA managed may or may not receive smart home technologies in their plan depending on the subjective assessment of planners on whether mainstream technologies are deemed as 'reasonable and necessary'. Often planners lack expertise and experience and resources to understand and provide tailored solutions for individual needs as it is (Laragy 2018). The NDIA, who administers the NDIS, has a responsibility to advocate for its participants and provide reliable information to enable participants to meet their needs in the best possible way. This includes being across new and emerging smart home technologies that are beneficial for participants (ADC 2019).

In context of the scale of funding increases as the NDIS becomes fully operational, from \$7.7 billion in 2017-18, to $\$ 14.5$ billion in 2018-19, spending on AT remains a minor component at below 5 per cent, and within this, smart home technologies are a marginal sub-category. Furthermore, if participants can secure smart home technologies, the complexity of what 'pool' of funding is used to support the upfront cost of the device versus service requirements (both upfront and ongoing) is unclear. As shown in Table 2, there are three support categories of funding (core supports, capacity building and capital supports) that participants can access for AT and home modifications. The limitation of the categories is their inflexibility; funding cannot be moved from one category to the other. For instance, although smart home technologies could be classified as capacity building supports, at present, they can only be funded under the core support or capital supports categories.

As an emerging field the smart home component of AT is yet to be clearly defined and established in government support program eligibility. For disability support through the NDIS, from October 2019 a new option for the funding and supply of nominated AT supports between $\$ 1,500$ to $\$ 5,000$ was introduced (NDIS 2019a). This funding option includes nine general AT items across four levels of AT needs complexity, with no explicit definition for smart home products. The overarching eligibility for technologies that are 'reasonable and necessary' is difficult to articulate for core items such as iPads or smart home assistants as these devices also integrate wider functions beyond the use of the needed AT. So, while these improvements target streamlining NDIS AT processes for low-risk AT under $\$ 1,500$, the eligibility for AT remains focused on physical non smart home types of AT. Smart home AT and home modifications are still not primary considerations in planning and are unclear in terms of the emergence of new products and the convergence of bespoke versus mass-market products with complex AT eco-systems. 
Table 2: NDIS support categories

\begin{tabular}{|c|c|c|}
\hline Core supports & Capacity building supports & Capital supports \\
\hline 1. Assistance with daily life & 5. Support coordination & 14. Assistive technology \\
\hline 2. Consumables & 6. Improved living arrangements & 15. Home modifications \\
\hline $\begin{array}{l}\text { 3. Assistance with social and community } \\
\text { participation }\end{array}$ & $\begin{array}{l}\text { 7. Increased social and community } \\
\text { participation }\end{array}$ & \\
\hline \multirow[t]{6}{*}{ 4. Transport } & 8. Finding and keeping a job & \\
\hline & 9. Improved relationships & \\
\hline & 10. Improved health and wellbeing & \\
\hline & 11. Improved learning & \\
\hline & 12. Improved life choices & \\
\hline & 13. Improved daily living & \\
\hline
\end{tabular}

Source: (National Disability Insurance Agency 2019).

\subsubsection{Aged care system}

The aged care system is complex and various levels of support are available. The Commonwealth Home Support Program (CHSP) provides basic entry-level aged care services to support sustained independence for people aged 65 and over living at home. In 2016-17, more than 720,000 people aged 65 and over received home support services (Australian Institute of Health and Welfare 2018). Care provided through the CHSP includes broad types of support to keep people well, safe, independent and connected with their communities. The intended outcomes of the CHSP are to ensure frail older people with functional limitations are supported to live in their own homes and have increased social participation and access to the community, including through the use of technology (Department of Health 2019). One of the CHSP sub-programs, known as Community and Home Support, contains 13 service types, two of which are relevant to this research: the Goods, Equipment and Assistive Technology and Home Modifications services. In the Goods, Equipment and Assistive Technology sub-program, seven sub-types exist ranging from self-care to car modifications.

Home Care Packages are an additional source of support for older Australians, offering access to affordable in-home care services. Commonwealth Home Care Package (HCP) provides a larger pool of funding (between $\$ 12,000$ and $\$ 50,000$ per annum) for people who may require higher levels of support and care beyond the entry-level program. There are four levels of support under the HCP program, which correspond to the complexity of care required with a set amount of funding ranging from Level One (low) to Level Four (high). The number of Home Care recipients increased by 84 per cent over the 10 years to 2017 to around 71,400 packages, including proportional increases in age bands above 75 and 85 years (Australian Institute of Health and Welfare 2018). This reflects the recognised strong preference of older Australians to age at home, in line with increased government program capacity to deliver community-based care. The number of Commonwealth HCP has continued to grow, reaching around 100,000 in 2018-19, with further increases planned in budget forward estimates to over 140,000 in 2019-20 (Australian Government 2018), while at the same time admissions into permanent residential care have decreased (Khadka et al. 2019).

As noted, it is increasingly well-recognised that more flexible support options have a role in reducing pressures on the aged care, disability and healthcare systems. While aged care service planning may not be the major concern of mainstream housing providers yet, the design of the home and the provision of smart home AT have direct cost implications for quality-of-life and care (Carnemolla and Bridge 2016b; Carnemolla and Bridge 2019). It also impacts housing decisions such as institutionalisation (Bridge et al. 2006) and downsizing (Judd et al. 2014). 
The complexity of the aged care system results in the person who carries out the AT assessment being determined by the scheme and the level of functional impairment. For example, the CHSP is intended to provide care for those with one or two service needs, whilst the HCP provides higher-level care. Both programs require an assessment process that is managed by two separate teams, the Regional Assessment Service (RAS) team reviews the CHSP, and Aged Care Assessment Team (ACAT) reviews the HCP. The RAS team is located across states and territories, with sub-contracted organisations who often sub-contract further, making it difficult to contact the relevant service provider. Like the challenges identified in the NDIS process, the ability to communicate with a consistent assessor that understand the needs of the participant creates barriers in obtaining funding and solutions that meet individualised needs.

The 2019 Royal Commission report on the aged care system also noted that changes experienced by the aged care sector were placing 'significant pressure' on the aged care workforce. It found these changes included the "increasing age of the population and aged care workforce, more diversity within those using aged care, increasingly complex health needs as people remain at home for longer, and the uptake of technology to assist with service delivery" (Aged Care Quality Standards 2019: 31). It noted the funding model for aged care had changed. Service delivery organisations were no longer directly funded by the Australian Government through 'block funding', and consumers were able to exercise greater control over how funding was spent. It also highlighted the rollout of the NDIS had added to pressures on the aged care workforce as demands for more staff grew across both the disability and aged care sectors (Aged Care Quality Standards 2019). Consequently, the responsibility has shifted from the state to the individual, assuming older people have the capacity to be across new and emerging technologies and exert self-advocacy for smart home AT.

Australian Aged Care policy reflects the objective of supporting people to be more independent at home. Including aiming to incorporate a safe, stable, and enabling environment; enhance quality-of-life, maintain, or improve functional status and/or psychological, emotional, and physical wellbeing. However, eligibility for smart home AT an emerging means of addressing these aims is lacking for smart home AT within Aged Care and evidence is unavailable regarding the extent of specific smart home AT product take-up under current policy settings. Smart home AT provides opportunities across all levels of home care packages as costs of specific products is already low and falling as these products are produced for mass markets globally (Hong et al. 2020; Schäuble et al. 2020). This is similar to currently available home modifications that include eligibility for supports such as the installation of handrails that are recognised to be effective and are relatively low-cost items. Thus, it is reasonable to suppose that lower cost smart home AT may be similarly effective for specific targeted groups.

At the national level, both the aged care system and NDIS present a complex and challenging maze to navigate, particularly in procuring the support required for smart home technologies. It appears there is little or no recognition of smart home technologies with all examples of AT given in the current policies pertaining to low level AT, rather than smart home technology.

\subsection{Existing research}

Existing research shows AT, such as traditional environmental control units (ECU) and telehealth care technologies, can increase independence and improve quality-of-life for older people and those living with disabilities (Noda 2018). However, mainstream smart home technologies that perform similar functionalities such as the Google Smart Home and integrated loT devices have limited evidence-based studies to understand the benefits, ease of use, cost-benefit analysis and real-life integrations because of their novel and emerging nature as an AT. Existing projects and studies on smart homes have focused on bespoke, one-off designs and systems that test the theory of an intelligent home or proof of concept. However, this lacks the applicability for mass adoption that off-the-shelf mainstream technologies provide. There is enormous potential for smart home technologies to be adopted as AT because the price point for devices is relatively low and the devices are easy to procure from a variety of technology providers (Layton and Steel 2019). More evidence-based research in real-life scenarios is needed to better understand the socio-economic benefits, particularly the psychological wellbeing impacts, and how people with disabilities and older people procure and maintain the technology. It is also important to ascertain whether smart home AT can enable and enhance independent living for longer. 
In the case of smart homes, the intervention is complex and crosses over multiple technologies that are dynamic and developing quickly. The actual devices are a physical focus, but the ubiquitous expansion of cloud-based platforms underpins low-cost integration of applications and devices and novel flexibility of how to build virtual smart home environments. The intersection and overlap between equipment, services and home modifications means the existing polices and funding guidelines limit the ability of participants to procure smart home AT to meet their individualised needs. The existing funding guidelines in the Australian aged care system and NDIS creates challenges for the type of smart home AT can and cannot be funded due to its categorisations, definitions and limitations on funding pools. All examples given in the existing policies pertain to low level AT and not smart home AT as further illustrated in Section 2. For instance, there is currently a lack of consideration on the required home modifications to successfully integrate smart home loT devices, particularly the requirement for NBN cabling and installation of fittings and fixtures.

The smart home AT market will continually grow over the next 10 years with a projected average of 37 Internet of Things (loT) devices per household in Australia by 2022 (Lee 2019; Zalunzny 2019). However, access to smart home technologies is not equal amongst all Australians. People with disabilities are often more socially-economically disadvantaged than people without disabilities (Kavanagh et al. 2015). When it comes to access to technology, the digital divide is apparent where the inability for people with disabilities to access Information Communication Technology (ICT) creates more disabling barriers (Macdonald and Clayton 2013). People aged 65 and over are also among the least digitally included groups in Australia, particularly if they are not living in a major city, are female or on lower incomes (Malta and Wilding 2018). Research shows that the older population can become proficient and have a positive attitude towards technology if training and information are provided (Kolodinsky et al. 2002). The Commonwealth in partnership with State and Territory governments and non-governmental funding bodies play a critical role in advocating for and enabling older Australians and those living with disabilities to access digital technologies that will prolong ageing in place and independent living. In particular, the increased availability and variety of smart home devices means there will be an oversaturated market and unpredictable rate for software and hardware obsolescence. People with disabilities and the ageing population need to be provided with best practice frameworks and accessible information that help them make an informed decision in a consumer-directed care model (Russell et al. 2020).

The pervasive nature of smart home AT also presents concerns of privacy and data security for vulnerable groups in society. Existing research in virtual assistants and smart home speakers show the intrusive nature and vulnerabilities to data hacking (Apthorpe et al. 2017; Bugeja et al. 2016). In instances where smart home technologies are being used as AT, it is possible for supposedly anonymised data to be deanonymised in several ways to identify real people. The increased risk of highly deanonymised data for very vulnerable people to be recorded and used is a big concern. End-users are often unaware of how the data is used, and the privacy settings on many smart home ecosystems are not tailored to privacy needs (Bugeja et al. 2016; Lau et al. 2018). Research shows end-users remain positive about smart home AT if they understand how the data is being used and the perceived benefit of the technology outweighs the privacy concerns (Courtney 2008). Nonetheless, the big dataset generated from the 'always on' monitoring of smart home loTs requires that data security and privacy legislation be introduced for the care and disability sector to safeguard vulnerable groups of society. Furthermore, the ability to monitor participants' movements and to access this at any given time raises ethical questions of consent and restrictive practices (lenca et al. 2018; Mahoney et al. 2007; Wangmo et al. 2019). The risks and liabilities are not clear as to who is responsible for what and the lack of standardisations that exist across the care and disability sector needs to be addressed.

Housing providers and the Australian Government (Martin 2020; HOI 2012) are positive about the potential smart home AT has for people with disabilities and the ageing population, with numerous new Special Disability Accommodation (SDA) developments incorporating smart speakers as a baseline offering. However, across the board, there are low-level adoption rates from end-users that could potentially be attributed to the lack of understanding or perceived usefulness of smart home AT. Unless the usefulness of smart home AT is clearly communicated, the likelihood of adoption and use declines, and consequently, negative attitudes build towards the technology (Eisma et al. 2004). Research shows the perception of usefulness and ease of use is increased if the end-user has experienced a form of smart home AT through either a family member or a friend (Ashraf et al. 2020). The full benefits of mainstream smart homes can only be assessed if participants begin to adopt the technology as AT. 


\subsection{The study}

This smart home AT project was undertaken in the context of both aged care and disability reforms. It aimed to improve consumer choice whilst maximising safety and independence in accommodation that is fit for purpose, and that augments loss of functional capacity. Currently, eligibility, access and out of pocket costs for AT provision in Australia differ depending on a person's age, level of disability, location, and importantly which service system is accessed. While AT offers many potential benefits in maintaining and prolonging independent living and improving quality-of-life, these benefits must be weighed up against the relative costs of care provision. For individuals with complex needs, where smart home AT integration may require substantive housing modification and/or where there is a forecasted need for ongoing adaptations and technology support, the total cost and uncertainty over both upfront and annual costs may result in smart home AT becoming cost-prohibitive or deemed as too risky. This research looks at the missing gaps to better understand the barriers in mass adoption of smart home AT from both the end-user perspective and that of care and housing providers to inform housing and funding policies. This includes an assessment of ease of use, quality-of-life, and cost benefit analysis.

This project contributes to the development of policy options that could facilitate effective adoption of smart home AT in Australia. The research is guided by the following questions:

- Research Question One: What are the current and emerging directions in the development of AT globally and nationally?

- Research Question Two: What are the current policy directions in disability and aged care in Australia and internationally for smart home AT?

- Research Question Three: What factors enable or constrain the use of smart home AT to support independent living in practice?

- Research Question Four: What are the potential economic and social benefits of policies and practices to support the development and adoption of smart home AT in Australia?

\subsection{Research approach}

The approach undertaken in this research was designed to deliver a better understanding of how a smart home control system (encompassing several diverse components working together as a unified portal or ecosystem) can benefit older people and people with disabilities with the intent to inform policy. A multi-method approach was utilised. First, the existing literature was used to develop a survey. Then, focus groups were conducted with disability and aged care housing providers and associated non-government organisations such as consumer and housing peak bodies. Following the survey, case studies were captured to illustrate the end-user experience. The firsthand data was combined with secondary sources for economic modelling.

\subsubsection{Qualitative analysis}

In order to provide an in-depth and detailed understanding of the impacts of new and emerging AT from the enduser perspective and explore the complexities involved in implementing the technology within the home, a mixed methods qualitative analysis approach was used. The methods included:

- Literature review: A literature review of the new and emerging AT in housing was collected and analysed. This overarching understanding was supplemented by a policy review of AT in care and housing provisions in New South Wales, South Australia, Tasmania and the Commonwealth. This review provided the basis for comparison with international practices and policies. (Research Questions One and Two)

- Survey: A user survey (see Appendix 1 and Appendix 2) of end-user satisfaction with ATs, its impacts on qualityof-life and procurement costs was designed and developed based on three parts (i.e. Assistive Technology; Quality-of-life Assessment and Demographic questions). Sixty key housing, ageing and disability non-government organisations were approached to distribute the user survey online; 20 national organisations did so. The invitation to participate was also promoted via social media. (Research Questions Three and Four) 
- Economic modelling: Modelling was undertaken based on data from three sources: first the user survey; second the information gained from a national AT supplier and lastly national census data from the ABS concerning population and functional impairment severity ratios. The economic aspect undertaken within this project is an important one as the data presented forms the foundation for a preliminary cost-utility analysis of smart technology value for policy. (Research Question Four)

- Focus groups: Five face-to-face and online focus groups were conducted across New South Wales (NSW) (n=11), South Australia (SA) $(n=6)$, Tasmania $(n=6)$ and Western Australia (WA) ( $n=1)$ with a combination of housing providers $(n=3)$, housing developers $(n=1)$, aged and disability service and housing providers $(n=13)$, insurance and care providers $(n=1)$, AT providers $(n=1)$, AT advisory services $(n=3)$, statutory agencies $(n=1)$ and end-users $(n=1)$. (Research Questions Three and Four)

- Case studies: Older people and people with disabilities who completed the survey volunteered to be interviewed to elaborate on their lived experience of, interest in and purchase of their smart home AT. The case study method, was used to collect these more detailed stories about the how's and why's of the sampled survey volunteers' smart home technology journey. (Research Questions Three and Four)

\subsubsection{Economic modelling}

The economic analysis draws upon the user survey to develop core smart home AT product and cost profiles incorporating trends, target populations and outcome scenarios. The economic component of the project integrates the literature review as well as findings from the focus groups and case studies. The combined data sources provide the basis to establish product set profiles and develop representative purchase, installation and maintenance samples to assess AT costs and Assessment of Quality-of-life (AQoL) outcomes in the context of funding sources and target populations as summarised in Figure 2. Sources from commercial providers and government funding of smart home AT for aged care and disability support provided supplementary validation to product profiles (dotted arrows) and potential model scenarios in the context of anecdotal rapidly growing trends in smart home AT. The core product profiles were integrated with economic modelling to examine preliminary cost-effectiveness estimates.

Figure 2: Economic data components for AT products

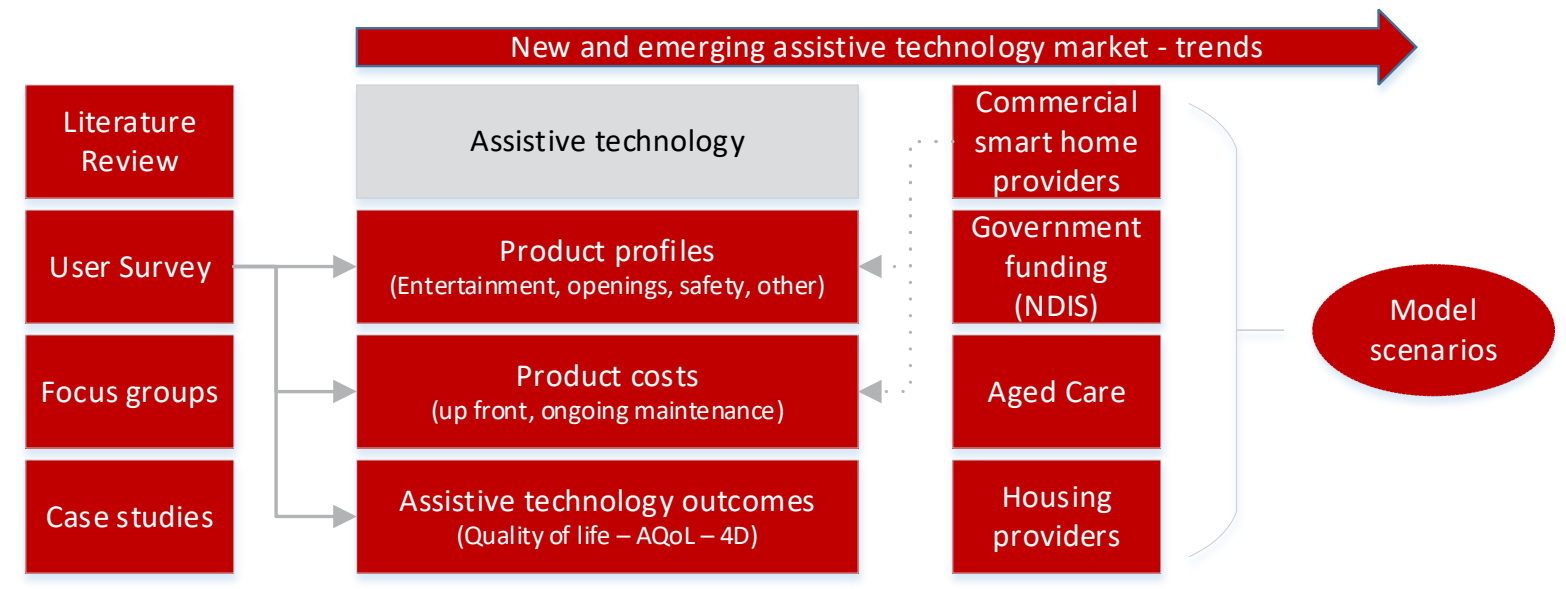

Source: Authors. 
Data sources

Our primary data source about smart home AT was our user survey (detailed in Section 3). The survey was distributed to relevant disability and aged care networks, as presented in Section 3.1. The survey aimed to provide perspective from consumers who have implemented assistive smart home technology including:

- AT products used

- Product cost ranges for purchase, installation and ongoing maintenance

- Funding source for private or government support

- Basic age group and demographics

- Assessment of Quality-of-life (AQoL-4D) before and after AT installation.

Smart home AT cost data was supplemented through consultation with commercial smart home retail groups to expand on product ranges and develop a profile of indicative AT installations for housing providers. Further pricing sources were also examined, including the NDIS price schedule for AT items.

As AT is rapidly developing, and consumer need and preferences are highly variable, the objective was to identify representative product and cost profiles over the expected useful life of typical installations.

AT profiles

The economic component of the project examined smart home AT profiles across cost categories including:

- Entertainment: smart tv, smart audio systems, automated lighting

- Openings: automated curtain, blind, window or door controls

- Safety: security camera, alerting reminder system(s), gas automatic shutoff, electricity automatic shutoff, water main automatic shutoff, smart smoke detectors

- Other: automated track hoist facilities, smart appliances, smart furnishings, assisted breathing device, home backup generator or battery.

AT costs

The survey included smart home AT cost items covering upfront purchase, out of pocket costs for governmentsupported devices, as well as annual ongoing maintenance. The survey stratified smart home AT purchase cost ranges across $\$ 200$ or less and bands to $\$ 500, \$ 1,000, \$ 2,000$ and above. The profiles were then also combined into two broad cost groups for below and above $\$ 500$. Each cost scenario has been collated from the primary user survey and smart home retailer data and supplemented from previously published research where available. All costs are presented in 2019 Australian dollars.

The research team also consulted with commercial smart home suppliers to develop typical assistive technology specifications for housing providers costings (Harvey Norman, 2019). This provided a representative smart home profile covering an example home automation system for an independent living and carer set-up for integration into apartments.

\section{AT funding sources}

Smart home AT is a newly emerging component of established Australian Government AT support programs that generally provide structural modifications or specific devices. As presented in Section 3.3.3, potential Australian Government funding is available across disability and aged care settings. The survey included items for governmentsupported funding and age above or below 65 years, the general eligibility points for disability transitioning to aged care. The remainder of the smart home AT market are self-funded or may have some level of Australian Government assistance integrated with self-funded components. 


\section{AT outcomes}

The survey data included the 12 question 'Assessment of Quality-of-life' (AQoL-4D) instrument to estimate health-related quality-of-life utility scores. The AQoL-4D was included in the survey as a short 12 multiple-choice questionnaire covering four AT relevant dimensions of independent living, mental health, relationships, and senses (AQoL 2014). As the project does not incorporate longitudinal follow up, the before and after AQoL questions were collected retrospectively at a single time point. Although this approach introduces additional limitations through potential recall bias, previous research utilising similar methods has demonstrated successful outcomes (Carnemolla and Bridge 2016a). The raw AQoL scores before and after AT installation were analysed in STATA v 16.0 (StataCorp LP, College Station, Texas, USA) using established algorithms to generate overall instrument utility scores and individual scores for the four dimensions scaled between zero (worst health state) and one (best health state).

\section{Economic modelling}

The AT costing profiles were developed into a basic Markov model framework as the basis of estimating costeffectiveness ratios, including indicative incremental cost per Quality Adjusted Life Year (QALY) gained. The modelling was undertaken for below and above $\$ 500$ smart home AT cost scenarios. Uncertainty in estimated model parameters was evaluated using bootstrapping resampling techniques of probabilistic sensitivity analysis. The economic modelling was developed in TreeAge Pro 2020 Release 1.0.

The modelling was not undertaken as formal outcome-based analysis, rather as a framework to examine the smart home AT cost data in terms of potential benefits over several years. This enables the cost-effectiveness to reflect the characteristic timing differences where smart home AT costs are undertaken substantially up front, with variation in maintenance and upgrade paths, while the resulting benefits are often dispersed over several years, including health outcomes that may be lagged.

\subsubsection{Limitations}

As the modelling has been undertaken primarily for gaining a perspective on a rapidly developing but still nascent smart home AT market, there are expected limitations to the analysis, data and modelling, including:

- The tight targeting resulted in a small sample size response to the user survey. As such, undertaking statistically significant and sub-group stratification modelling based on age, location, culture, and so on was problematic.

- The smart home AT market currently receives negligible funding through Australian Government support programs for aged and people with disability, limiting the potential to evaluate smart home AT as an emerging support component.

- The rapid growth in the market can be attributed to predominantly private spending, with limitations on commercial sales data and products.

- Potentially significant areas of benefit, such as reduced falls, undetected falls or delaying quality-of-life decline, are not included in the modelling as data specifically related to smart home AT in this area is not currently available.

- The expected quality-of-life improvement from smart home AT implementation will erode over time as an individual's strength, health and mobility decline with age. Smart home AT devices may slow or delay this decline. However, this remains a complicating confounder in evaluating the cost-effectiveness of smart home AT across progressively older age groups.

- Definitions and smart home AT eligibility criteria are subject to interpretation, resulting in uncertainty of where products may be Australian Government funded.

- The survey combines user reported before and after AQoL responses at a single retrospective timepoint as longitudinal follow up is not in scope for this project.

- Survey questions were optional resulting in variation in response rates for separate questions, noted where relevant throughout the report.

- The market is highly diverse, with characteristic high variation in product profiles and specific need of target populations and therefore consumer heterogeneity limits generalised conclusions on smart home AT overall. 
The rest of this report is divided into several chapters as follows:

- Chapter two sets out to answer the question of what are the current and emerging directions in the development of AT globally and nationally? This is achieved by reviewing the existing academic and grey literature. A concise review of key trends and funding initiatives is presented to provide a contextual background to establish the relevance of the research undertaken.

- Chapter three sets out to answer the question what can we learn by asking end-users about their smart home AT purchases and outcomes? The statistical aggregation of responses from people with disabilities and older peoples based on personal experiences with smart home AT partially answers Research Questions Three and Four. In providing an overview of the results of the user survey, the process for design and development is provided as background. This is followed by a detailed analysis of respondents' demographics, in the context of their smart home AT devices and services experiences. A structured, statistical analysis is combined with some discussion on the implications for government policy towards smart home AT.

Chapter three also sets out to answer the question What are the potential economic and social benefits of smart home AT policies? It reviews the economic perspective for smart home products reflecting the characteristic nature of this emerging market segmentation with high variation in product ranges, specifications, functionality, quality and cost. The overarching perspective is a rapidly growing smart home market utilising new cloud-based technologies and increasing potential target populations across aged care and disability settings.

- Chapter four sets out to answer the question what can we learn by asking end-users about their smart home AT purchases and outcomes? The focus on housing providers and non-government organisations from the five focus groups combined with the four case studies further elaborates on Research Questions Three and Four, reporting a more nuanced and individualised experience.

- Chapter five sets out to answer the question of what policy and practice implication can best inform future work? It elucidates the issues associated with policy development in a complex space where the technology itself is rapidly evolving and where current guidance and policy is at best confusing, and at worst, absent altogether. This chapter summarises the issues and responses to implications of new and emerging AT for older people and those living with disability and recommends some policy and practice changes that may improve outcomes. 


\section{Smart home assistive technology}

- Smart home AT has the potential to be incredibly beneficial in promoting independence, self-care and ageing in place with increased safety and quality-of-life. However, more evidence-based research is required to understand the long-term effects and cost benefits.

- Globally, smart home AT is seen as a transformational provision for people living with disabilities and the ageing population. However, the ratification of the United Nations Conventions on the Rights of Persons with Disabilities has been slow, in particular, there is a lack of monitoring and assessment of policy implications.

- The need to standardise AT provision and develop criteria for assessing process and service models underpinning smart home AT products and services is a global problem.

- There is currently a lack of research considering the required home modifications to successfully integrate smart home loT technologies, particularly the requirement for NBN cabling and installation of fittings and fixtures.

- The smart home loT market will continually grow over the next 10 years, with a projected average of 37 loT devices per household in Australia by 2022.

- The increased availability and variety of smart home devices at more affordable prices is beneficial for older Australians and those living with disabilities. However, the increased growth of product and service availability means there is an unpredictable rate of software and hardware obsolescence, creating challenges for end-users in a consumer-directed care model. 
- There are concerns of unethical practices involving privacy breach and potential for restrictive behaviour controls that can occur when the social and behavioural dimensions are constantly monitored and analysed.

\section{- There are also concerns around data security and privacy of smart home AT that calls for a review of national legislation with a focus on the care and disability sector who are dealing with sensitive personal and identifiable data.}

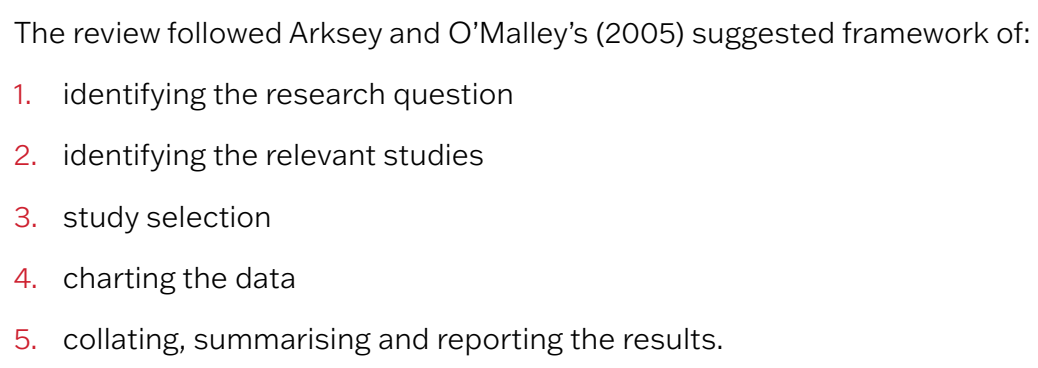

The review followed Arksey and O'Malley's (2005) suggested framework of:

1. identifying the research question

2. identifying the relevant studies

3. study selection

4. charting the data

5. collating, summarising and reporting the results. the current and emerging directions are in the development of smart home AT globally and nationally. A scoping review method was employed as a way to rapidly understand the key concepts and broader nature of smart home AT and its policy implications whilst providing in-depth information. The scoping review followed the suggested framework by Arksey and O'Malley (2005) for academic rigour and replicability. The iterative nature of a scoping review was also advantageous for a topic area that is emerging in a variety of disciplines and rapidly evolving.

First, the research question was developed as part of the initial project proposal: What are the current and emerging directions in the development of assistive technologies globally and nationally? And What are the current policy directions in disability and aged care in Australia and internationally for smart home AT? An initial search protocol was developed to identify relevant studies for the research questions. A search on the following key databases: Scopus, PubMed, PsycINFO; Web of Science; and Google Scholar was conducted with the keywords and Booleans listed in Table 3. The search was restricted to articles written in English and published between 1990 and 2020. The study selection included both qualitative and quantitative studies to encompass the breadth needed to understand the emerging nature of smart home AT. Any duplicated studies were eliminated. Each publication was reviewed for its relevance, application and scholarly rigour in relation to the project aims and research questions.

Table 3: Search terms

\begin{tabular}{llll}
\hline Database & Key search term & Key disability term & Key ageing term \\
\hline - Scopus & - Smart Home & - Disability & - Ageing \\
- PubMed & - Smart Technology & - Disable & Older \\
- PsyclNFO & - Assistive Technology & \\
- Web of Science & - Domotics & \\
- Google Scholar & - Intelligent Home & \\
& - Automated Home & \\
\hline
\end{tabular}

In the search, "key search term" with "key disability term" and "key ageing term" was entered with the and/or Boolean function. Source: Authors. 
Whilst traditional academic literature informed the theoretical frameworks of smart home AT and provided insights into the historical developments, there were limitations and opportunities to its application. The literature search resulted in very limited information on policy developments and global applications of smart home AT for the ageing population and people with disabilities. Furthermore, the results of the traditional academic literature search demonstrated a heavy focus on technology developments that were specific to a product or system and was situated within the field of computer science. For instance, Obaid et al. (2014) look at Zigbee wireless voice control systems for people with disabilities and older persons with a specific focus on the voice recognition system and wireless system configurations, rather than usability and home integration. As a result, we needed to supplement traditional academic literature with a grey literature review to answer the second research question. In order to understand the global trends, emerging technologies and policies critical to this research project, a general search was conducted with the same keywords as the academic literature review with the addition of the keyword 'policy' added. The search was also supplemented by the expert knowledge of the research team on current developments and policies internationally and nationally. The grey literature included was reviewed for its applicability and relevance to the research questions, the reliability of the source, and its policy implication over time.

From the scoping review, we can see that globally, the rise in ageing population needs new and innovative ways to prolong independent living to help healthcare systems cope with growing demands for care (Francesca et al. 2011; Health and Health 2006). AT is known as an effective way to help improve independence, promote social connection and enable 'ageing in place' (National Aged Care Alliance 2018; Song and Van Der Cammen 2019). In Australia, one in every six Australians is aged 65 years and over, and over half of all older Australians have a form of disability (ABS 2018b). It is predicted in the next 20 years the number of Australians over 85 and those living with chronic and multiple functional impairments is going to double (ABS 2018). AT can be a cost-effective way to not only prolong independent living but also improve the quality-of-life for older people and those living with disabilities (National Aged Care Alliance 2018; Song and Van Der Cammen 2019). People with a disability use a variety of aids or equipment depending on their physical limitations and environmental factors to assist and support their ability to remain in their own homes. Layton and Steel (2019) proposed that new and emerging commercially available mainstream smart home AT would be cost-effective for people with disabilities better enabling them to live independently and to 'age in place'.

Ageing population growth is a global concern (Khan 2019; Li et al. 2019). As such, many countries are grappling with how AT might be a key component in improving quality-of-life and alleviating the demands on care systems by prolonging independent living (WHO 2018). Although older people with impairments are generally considered a social norm, they are rarely regarded as 'disabled' in the same way as young adults with disabilities might be (Priestley 2002). As a result, often older people and people with disabilities receive separate approaches in policy making, service delivery and activism (Leahy 2018). It is important to note this key paradox as it provides distinct funding entitlements, as well as service and procurement procedures, of smart home AT in Australia for older Australians and those with disabilities.

For people with disabilities, AT provisions have existed in many countries for many years. Most recently, the United Nations Conventions on the Rights of Persons with Disabilities (CRPD) has become an instrumental treaty, introducing an international legislative obligation to treat people with disabilities as equal members of society and facilitate access to AT solutions (De Witte et al. 2018). More than 170 countries, including Australia, have committed to the ratification of the set standards. Ratifying countries have an obligation to design policies and legislation that ensures access to support services (including affordable AT) for all persons is viewed as a fundamental human right. The AT provision here refers to both devices and any services required for the AT to function fully. 
The CRPD has been an instrumental propeller for policy developments. However, the implementation and uptake has been slow, leaving a considerable gap between the need for, and access to, AT (Maalim et al. 2019). The WHO figures suggest that only 10 per cent of the 10 billion people who need AT have access to it (Maalim et al. 2019). The initial two-year reporting indicated that most ratified countries have systematically embedded AT into policy. However, they lacked evaluation and monitoring procedures to assess the effectiveness of the policies (MacLachlan et al. 2018). As a result, the Global Cooperation on Assistive Technology (GATE) was established in 2014 to help propel AT provisions and improve access. Additionally, WHO commissioned the Global Report on Assistive Technology steered by the GATE, UNICEF, ad-hoc AT experts and WHO Secretariat. To progress the report, the first Global Research, innovation and Education in Assistive Technology (GREAT) summit was held in August 2019. The summit canvassed current needs, demands and supply of AT, invited good practices and innovation, and identified gaps for improved access (WHO 2019).

Globally, AT provisions is an area that needs to be improved upon, including the supply, access, outcomes, policies, and procurement process within complex healthcare systems. The availability of mainstream technologies, such as smart home IoT devices and smart phones, is seen to be revolutionising the perception of AT, access and affordability (Disability Federation Ireland 2016). It is recognised that AT addresses a variety of user groups and the wide range of available AT products and related services means the system is complex. This complexity is further compounded by the fact that the impact of an AT solution depends largely on the aspirations and individual characteristics of the user (De Witte et al. 2018). A one stop solution that fits all is rarely the case (De Witte et al. 2018). In addition, the rate of technology developments and new products, suppliers and service providers entering the market is rapid. As a result, this exasperates the need for robust policies and nimble systems that address the end-user's needs.

The following is a summary of some of the current global trends on AT provisions and policy developments that address the complexities of procurement, access and outcomes in a successful or innovative way. The global developments and concepts are canvased for the purpose of discussion and its potential consideration in informing Australian housing and disability policies.

\section{AT passport concept}

In 2016, the Disability Federation of Ireland and Enable Ireland prompted a discussion on the fragmented and underresourced provisions for AT, advocating for a focus on AT infrastructure. The concept of an AT health passport was tabled as a way to deliver user focused AT provisions with centralised coordinating agencies to address the issues with comprehensive service provision and innovation. The AT health passport would record the history of equipment, training and funding. It would act a central platform to facilitate coordination between multiple funding agencies, streamline procurement process and deliver contextualised and accessible information to the end-user on the newest AT (Disability Federation Ireland 2016). There was a particular emphasis on AT that was mainstream, or specialised AT that was affordable, up-to-date and appropriate to support individual needs.

Ireland launched the 'Health Passport' in June 2019, focused particularly on improving care for those living with intellectual disabilities (Condon 2019). The Health Passport is a document that contains vital information about the participant, including personal details, medical history and communication abilities. The project aims to provide a platform for the participant's voice to be captured and considered in the assessment and provision of any services, including AT. The launch of an AT specific passport has yet to occur. However, the idea was raised in the 2019 GREAT summit by Maalim et al. (2019). The concept is particularly important in ensuring end-users are enabled through choice in a typically closed market where decisions are usually made by intermediary bodies like insurance companies (De Witte et al. 2018). Autonomy and choice are critical in the long-term use and adoption of AT. However, this has to be supported by advocacy, vetting and knowledge exchange to help end-users make informed choices. 


\section{European Assistive Technology Information Portal (EASTIN)}

The rapid speed of technology developments means more products and service providers are entering the AT market. This creates difficulties for end-users, healthcare providers, assessors and family members to understand the wide range of products available to make an informed choice. Established in 2005, the European Assistive Technology Information Portal (EASTIN), provides a unified database and search engine to deliver reliable information on AT. EASTIN amalgamates six national databases to provide access to over 60,000 products in the European market and approximately 20,000 companies that manufacture, supply or sell AT (Gower et al. 2012). In addition to a searchable database, EASTIN also provides collateral information and fact sheets. The EASTIN search engine has become the European landmark for AT information and is gradually increasing its coverage to ensure information is available to support service delivery (Andrich et al. 2013).

Although Australia has the National Equipment Database (NED) to browse and search for AT, the lack of new and emerging smart home AT products in the database is a concern. In addition, the products are grouped by topics related to task performance only. There are limited filtering options for price points. The nature of smart home AT is interconnected to multiple tasks within the home and requires a systematic approach rather than a piecemeal clustering of ad hoc devices.

\section{Nordic model}

Nordic countries have a long history of cooperation in AT, providing a publicly funded system that puts the end-user at the centre of AT provisions. For example, in Norway, the provision of AT operates using a team approach. Different services and sectors cooperate to achieve a holistic solution for individual needs, allowing users to try AT before purchasing. The local municipalities, regional and national authorities have a clear framework of responsibility for the provision of AT. The local municipalities are responsible for the direct health and rehabilitation of the end-user, whilst at the regional level, AT centres provide common resources and expertise (Sund, 2017). The clear articulation of responsibilities and focus on providing expert knowledge and reliable information builds a foundation for good practices and efficient resource management (Suzurikawa, et al. 2021). The decentralised approach of the framework delivers a user-centred solution that tailors AT provisions for individual needs. New and emerging technologies can also be captured through by dedicated AT centres that are responsible for innovation, best practice and knowledge dissemination.

As a result, Nordic countries are more progressive in exploring smart home AT. Finland has been leading the exploration of smart home AT solutions for some time now. One of its notable projects on 'Good living at home of elderly people' implemented from 2005 to 2008 (Melkas 2013) looks at a smart home pilot across three regions. The project aimed to build technological solutions and new service support models for elderly people (Melkas 2013). Innovations mentioned included incentivising competing providers to create more user choice and direction in the market. Also, a greater focus on quality assurance through accreditation and monitoring and standardisation of processes (Colombo and Murakami 2013). The articulation on who pays, and who is responsible, is clear. Finland is also harnessing the power of affordable mobile devices to help elderly people remain connected to their communities. For example, regular virtual lunch groups are held through video conferencing and prescribed mobile tablets create a sense of community at affordable prices (The Economist, 2020).

\subsection{A call for international framework for AT provisions}

Given the complexities in AT provision, a call for an international framework was canvased in the first GREAT summit in August 2019. De Witte et al. (2018) argues that an international standard will have a big impact on the accessibility of AT for people with disabilities. The key themes identified during the GREAT summit that need to be addressed include:

- A market that provides and leverages affordable high quality AT devices and services

- Informational systems that provide clear communication on what AT exists, supported by professional experts and service providers 
- Funding mechanisms that address users' individualised needs with transparency and clear pathways of funding allocations and responsibilities

- A quality assessment system for AT service provisions using the Association for the Advancement of Assistive Technology in Europe (AAATE) criteria. The seven key criteria are accessibility, competence, coordination, efficiency, flexibility and user influence.

The global trends and developments above provide an understanding of existing policies and frameworks within Australia. The following section will look at the current definitions of AT and the Australian funding context.

\subsection{Current trends in smart homes}

In the last few years, the key device that has stimulated the global smart home market is the smart speaker with virtual assistants (Markit 2019). Google, Amazon and Apple have all developed their own models. Smart Speakers were first introduced into the United States (US)in 2014 and, 12 months later, arrived in Australia (LERA 2019). Through a voice prompt, users can trigger Google to retrieve information, perform tasks such as turning on a TV, and control various parts of their physical environment. Amazon Echo and Apple HomePod offer similar functionalities in combination with third-party smart speakers with virtual assistant integration such as Bose or Sonos. It was forecasted that by the end of 2019, 26 per cent of all Australian households will be using a smart speaker (Lee 2019). In 2018, the Australian loT at home (IoT@Home) market grew by 57 per cent to $\$ 1.1$ billion, with over 50 per cent of all Australian households owning at least one loT@Home device (Lee 2019). It is predicted that the average household in 2022 will have 37 devices, with half being loT@Home (Lee 2019; Zalunzny 2019). Globally, the loT market is set to reach $\$ 1.6$ trillion in 2025 (ABS 2020). Although, anecdotally, the smart home market in Australia is growing rapidly with declining product prices, there are no official data sources available to examine the size, composition and growth trends in this AT global market segment.

\subsubsection{Device and service market}

The growth trajectory of smart home devices is increasing consumer choice. However, the growth rates also mean there is potentially a large and confusing marketplace with too many choices for consumers. In a consumer-directed care approach, access to accurate and reliable information is critical. In the study of consumer experiences of home care packages, Russell et al. (2020) found participants also require ongoing information about the variety of services and entitlements on offer as their circumstances change (Russell et al. 2020). The nature and pace of smart home technology developments evolve rapidly as new technologies are introduced into the market. The rate of product obsolescence increases the pertinence of consistent communication. In addition, the smart home market is novel with many kick-starter products and options that require providers to deliver timely communication, play a role in helping standardisation and establish best practice across the sector. This process should involve co-assessment with the end-user, their supports and the care provider to achieve the best possible results. Research shows the success of AT requires an integrated approach from the design of the physical environment to the inclusion of care provisions as a holistic solution (Dewsbury et al. 2001). The plethora of choices will require best practice frameworks and rich knowledge exchange across disciplines to best leverage smart home technologies as AT.

As the smart home market is still emerging, the speed of technology obsolescence is not clear. Phillips announced it would cease all online support for the first generation of Phillips Hue lights as of April 2020 (Crist 2020). Users of Phillips Hue V1 lights will still be able to control the light locally via the Hue App. However, the lights will not be able to access the internet or upload security standards (Crist 2020). Smart homes rely on an ecosystem of technology such as smart phones and tablets that play an essential role in providing full control of the home for end-users. Technology usually has planned obsolescence (Richter 2019; Rossignuolo 2020). This means that early adopters of smart home devices will face challenges of more rapid technology obsolescence (Meade and Rabelo 2004). In addition, the emerging nature of the smart home being used as AT for people with disabilities and older people means there is very limited understanding of the full impact of technology obsolescence for both care and housing providers and participants (Layton and Steel 2019). Also absent is a transdisciplinary understanding of best practice frameworks that integrate interdisciplinary perspectives including but limited to designers, allied health care provider, and funding bodies. Consequently, while numerous trial projects exist, the technology systems employed in each project vary, and results are unable to be cross-validated (Liu et al. 2016). 


\subsubsection{Reliance on periphery services}

The interconnected nature of the smart home ecosystem means it relies on wired infrastructure and stable and speedy network for devices to communicate with each other and to the cloud. In Australia, the rollout of the National Broadband Network (NBN) and 5 G network is set to enable more smart home technology adoption. Delays have occurred with the NBN rollout and the process has been challenging. The NBN rollout schedule has been criticised for the social discrepancy across status of the release sites (Alizadeh 2013). In a study that compared six of the earlier rollout sites to the ABS Census-based socio-economic indexes for the areas (SEIFA), it is evident that the network infrastructure is heightening the divide between social groups (Alizadeh 2013). Although some regional and remote locations benefit from the NBN rollout schedule, the most distressed and isolated localities at the bottom of the SEIFA index could fall behind even further (Alizadeh 2013). This additional digital divide compounds the inequalities that exist for people with complex needs, psychological and social disabilities, those from Aboriginal and Torres Strait Islander communities, people living in remote and very remote communities, and people from culturally and linguistically diverse (CALD) backgrounds in obtaining the support required through the NDIS (Malbon and Carey 2019). People in these categories would also benefit the most from AT through increased independence, community integration and improvement in quality-of-life.

Consumer choice is heavily dictated by the availability of services and infrastructure, and the ability to procure funding and decipher the best product option. The level of complexity in understanding the best choices is incredibly difficult in a novel market. Although the idea of a market-based policy framework is to provide choice and control for participants and stimulate market competition, the difficulty for people with disabilities and older people is the ability to navigate the complex web of competitors with limited knowledge and guidance. The smart home requires effective and usable integration of devices and services. As explained in the next section, raising considerations of the actual quality of the devices, operational life, interoperability and usability as well as the maintenance, ongoing support and installation needs across different providers needs to be cohesively simplified and accessible for participants.

\subsection{Smart home as AT}

The smart speakers allow users to control smart devices with voice alone. Unlike previous environmental control units (ECU), the inbuilt language processing software can recognise natural speech and many lexicons. For older people and those with disabilities, smart speakers can replace traditional ECU to assist with daily tasks, increasing ease and safety (Noda 2018). As the products are designed for mass consumption, they are also widely available and relatively affordable, with diverse options that can be tailored to individualised needs. Although the technology can be customised to a degree, people with more complex needs still require modifications outside the manufacturer's settings to achieve the same operable functionalities (Stefanov et al. 2004). The smart devices currently available allow users to control a variety of tasks within the home, such as indoor temperature control, opening blinds and doors, turning lights on and off, adjusting table heights, and automating water shutoffs. Table 4 provides more examples of the type of smart devices and the automated tasks that are beneficial for older people and those living with disabilities. 
Table 4: Examples of smart home technologies

\begin{tabular}{lll}
\hline Safety & Entertainment & Other \\
\hline - Security camera / CCTV & - Smart tv & - Automated track hoist facilities \\
- Alerting reminder system(s) & - Smart audio systems & - Smart appliances (including kitchen \\
- Gas automatic shutoff & - White noise control system & - Smart furnishings \\
- Electricity automatic shutoff & - Automated lighting & - Assisted breathing device \\
- Water main automatic shutoff & & - Home backup generator \\
- Smart smoke detectors & & \\
\hline & & \\
\hline Thermal & Openings & \\
\hline - Automated air conditioning & - Automated curtain/blind control \\
- Automated fan & Automated window control & \\
& - Automated door control & \\
\hline
\end{tabular}

Source: Authors.

\subsubsection{What are the benefits of a smart home?}

It is globally recognised that AT and environment interventions, such as home modifications, prolong independent living (Mann 1999). For older people and people with disabilities, key benefits include:

- Prolonging 'Ageing in Place' and alleviating the need for institutionalisation; most older people feel comfortable staying in their own homes where they are familiar with the environment and connected to their existing community (Khosravi and Ghapanchi 2016) in a safer and more secure environment (Alliance 2018).

- Improving the capture of critical information through remote monitoring of health status to identify and bolster functional abilities and overall wellbeing (Scherer and Glueckauf 2005).

- Enabling more evidence-based decision making for users and family members on future health care plans. The data collected from smart home devices can be analysed and accessed by the user, family members and health care providers to facilitate a person-centred decision-making process (WHO 2018).

- Helping users remain connected to the community and outside world, with the potential to reduce feelings of isolation (WHO 2018).

- Potential for substantial cost savings by improving quality-of-life and reducing care provisions (Carnemolla and Bridge 2016b; Lansley 2004),

Previous research into AT and home modifications has also and ultimately allowing people to age within their communities in a However, whether smart home AT can reduce the need for support services, and to what extent, remains unclear. This lack of clarity arises because existing research is limited and mostly concerned with falls and older people, and the findings from different research studies vary. Furthermore, technological developments are often too rapid to fully capture in traditional scholarship, which means the ability to integrate innovations into a range of smart home AT is poorly understood and implemented in practice (Thompson et al. 2012). 


\subsubsection{Effectiveness of smart home AT}

The effectiveness and ease of use of smart home AT is unknown, with limited studies investigating the end-user perspective such as user satisfaction. For example, the systematic literature review conducted by Brandt et al. (2011) on activity, quality-of-life and user satisfaction of smart homes resulted in two studies with evidence of higher user satisfaction, but low sample sizes and this result has been confirmed by more recent studies. However, the number of reliable studies focused on the end-user perspective remains low. A systematic literature review conducted by Morris et al. (2013) on smart homes for older people living at home also identifies the lack of depth and variation. Very small sample sizes and lack of clear definitions regarding inclusion criteria remain a problem in developing a robust discourse and set of policy expectations. As a result, the existing literature has very limited targeted studies on mass consumer technologies such as Google Home (Thompson et al. 2012). Although this is the case, we can see that studies conducted on 59 participants of the Slovenian Smart Home IRIS (Independent Residing enabled by Intelligent Solutions) project illustrated an increase in functional independence, better performance and satisfaction when living in a smart home fitted with the latest technologies available (Ocepek et al. 2013).

Although many industries and government initiatives have piloted projects in exploring smart home AT, these pilots have focused on developing new systems or proof of concept for unobtrusive sensors (Karunanithi and Zhang 2018), health monitoring and ad hoc automation systems (Department of Communities, Child Safety and Disability Services 2012) with little limited replicability. As mainstream smart home technologies converge with traditional ECU, more replicable and long-term research is required to increase reliability and effectiveness of smart home technologies (Layton and Steel 2019).

The introduction of new smart home technologies has an advantage over traditional ECU that is not often prescribed to participants because of user concerns with the high costs, the need to upskill to use the devices and a negative response to the aesthetic appearance of the devices (Holme et al. 1997). New smart home AT is less obtrusive. A Google Home Pod is less than $150 \mathrm{~mm}$ tall and $100 \mathrm{~mm}$ in diameter and promises an easy 'out of the box' experience. However, there is limited evidence assessing the ease of use and out of the box experience for older people and people with disabilities. Most studies on the effectiveness of smart home AT have been conducted in an environment whereby the AT is already set-up for the participants (Morris et al. 2013). A better understanding of end-user perception and the procurement journey from purchase to use is important for policy development. This understanding will also help care professionals to appropriately prescribe smart home AT as part of a long-term healthcare plan, aged care plan or plan for people with disabilities. The effectiveness of smart home AT is reliant on a holistic approach in the planning, procuring and delivery process (Ocepek et al. 2013).

Current research into smart home AT is varied in scope and diverse in objectives, involving predominately researchers in the field of information computing, human-computer interaction, health, and social care (Brandt et al. 2011). For example, the objectives of previous research can be broadly categorised by its intended services of providing comfort, healthcare and security (Alam et al. 2012), human computing interaction theory (Saizmaa and Kim 2008) to data acquisition by sensors and inference of knowledge through data-driven approaches (Litz and Gross 2007). The primary focus is how the technology works and proof of concept, rather than integration into the physical dwelling.

\subsubsection{Dwelling design considerations}

It is unsurprising that many disciplines are looking into smart home AT. Inherently, smart home AT encompasses different disciplinary knowledge involving people, technology and the environment. However, it is concerning that limited research has been conducted from the built environment discipline; smart homes directly impact the way dwelling typologies should be designed and considered with the inhabitant in mind (Carnemolla 2018). Technology integration needs to be considered during the conceptual stage of the design process to enable the best possible design solutions. For smart home AT, the intersection with the physical dwelling environment poses questions on the extent of home modifications that may be necessary to implement the system successfully. For example, the rewiring of electrical and data connections is required if existing cables cannot provide the speed and bandwidth required for loT connectivity. In addition, smart home AT may require home modifications, such as new fixtures and fittings on walls and ceilings beyond the plug and play of a smart speaker. 
Furthermore, an evidence-based approach is necessary from the perspective of the built environment to develop reliable and tested guidelines for construction codes and standards. The NDIS released the first Specialist Disability Accommodation (SDA) Design Standards in October 2019. The Standards aim to improve the clarity of design requirements for new SDA sites to raise the confidence of providers around compliance with the National Construction Code (NCC). The SDA Design Standards edition 1.1 contains very limited recommendations for smart home automation. Home automation is mentioned in: Section Two - Pedestrian entry from the site boundary; Section Four - Entrance, Doorways and Door Hardware; and Section Six - Windows. However, the only recommendation is that cabling, and power are supplied for future automation rather than smart home automation from the outset. The clauses are only applicable for "Fully Accessible" and/or "High Physical Support" SDAs. Appendix Six lists all instances of home automation raised in the NDIS SDA guidelines. AT is mentioned as a separate category with two clauses. Clause 23.1 stipulates high-speed internet must be provided with reliable and stable Wi-Fi coverage throughout all areas of the dwelling. Clause 23.2 requires a video, intercom or other communication systems to be provided for the purpose of communication between participants and their supports.

Although the first edition of the NDIS SDA Design Standards contains very limited clauses on the inclusion of smart home AT or provisions to enable future smart home integrations, it does include an appendix that sets out the general best practice recommendations, including home automation. In Appendix A of the NDIS SDA Design Standards, home automation is recommended for: height-adjustable benchtops in kitchens; smart devices to operate heating and cooling; automation of sprinkler systems for all dwellings with more than two participants; and power supply for sanitary facilities for future sensor operation of taps, toilet and lighting. The recommended best practice clauses provided in the NDIS SDA Design Standards are a positive step towards inclusion of smart home technologies in all SDA new builds. This reflects of a positive attitude towards new and emerging AT and recognises their potential for increasing independence and liveability.

\subsubsection{What are the risks of smart home AT?}

Smart homes can improve quality-of-life and allow older people and those with disabilities to live independently for longer (Ocepek et al. 2013). However, there are risks involved in making a home 'smart'. The smart home ecosystem is inherently reliant on the ability of devices to be context-aware and communicate with one another. The data logging and exchange generated by the ecosystem presents concerns on privacy, security and ethical propositions. These concerns need to be carefully considered before introducing smart home as a sustainable AT solution. Ethical concerns have been observed to be a major predictor of successful technology adoption. If ethical concerns of end-users and their caregivers are not adequately addressed, this often leads to scepticism and distrust of the technology (lenca et al. 2018).

Since these technologies are to be used by vulnerable individuals with physical frailty or disabilities and cognitive disabilities, the capacity to provide consent to their use is a big ethical concern (Wangmo et al. 2019). In particular, people with cognitive or psychologically related disabilities such as dementia could be taken advantage of if there are no legislative and policy safeguards (Mahoney et al. 2007). Research shows that the most frequently cited ethical concern for people with cognitive impairment is the clarification of informed consent (Mahoney et al. 2007). This area needs additional research to understand the required safeguarding measures.

The following concerns have also been identified:

\section{- Risk of data privacy, security and unethical practices}

The risk of data privacy, security and abuse of vulnerable groups is a big concern when it comes to smart home IoT devices that have always-on monitoring features (Wangmo et al. 2019).

\section{- Data vulnerabilities and management}

The data vulnerabilities and lack of transparency on how data is managed, stored, and protected is an increasing concern. Passive network observers, such as Internet service providers, could potentially analyse loT network data to infer sensitive data (Apthorpe et al. 2017). 


\section{- Privacy and consent from secondary users}

When smart home loT devices are used, privacy tensions arise when the use extends beyond the primary user to care providers. Secondary and incidental users who visit the dwelling may be unaware of the smart home IoT devices in play (Lau et al. 2018).

\section{- Care replacement}

As smart home AT becomes increasingly promoted and adopted, the ethical considerations of how this will potentially reduce or otherwise impact formal face-to-face care provisions is a concern for both users, and family members (lenca et al. 2018).

\section{- One size fits all assumption}

Although mass consumer smart home AT can be seamlessly integrated into the home, making participants feel like they are living in a standard dwelling (Amiribesheli et al. 2015), there is a risk in assuming the out of box experience fits all. Individual aspirations and goals of end-users need to be considered for any AT provisions (Eisma et al. 2004).

A key barrier to the adoption of new and emerging AT is the scepticism of the usefulness of the technology and fear of technology being used as a replacement for human care that would reduce the quality-of-care provisions (Lindqvist et al. 2013). Research shows that concerns for the user's perceived usefulness of the technology can overcome privacy concerns (Courtney 2008). For example, older adults reported to readily adopt smart home AT if it increased their independence and function if privacy concerns were addressed (Morris et al. 2013). It is important that any policy frameworks address these concerns explicitly to protect the most vulnerable groups in society from any unregulated behavioural modifications and seclusions (Chandler et al. 2014).

\subsubsection{The smart home AT market}

Although anecdotally the smart home market in Australia is growing rapidly with declining product prices, publicly available data outside of individual company annual reports and telco smartphone analysis is unavailable. Consequently, it is impossible to determine the size, composition, and growth trends categorically in the smart home AT market segment in harder economic terms. However, according to a leading consumer advocacy group in Australia the average household had nine connected devices in 2015, increasing to 17 in 2018, with continued expected growth to reach 37 by 2022 representing a four-fold increase in seven years (Zalunzny 2019).

In the case of smart home AT devices delivered to older people and people with disabilities specifically, the costs are often borne by either or both Commonwealth/State Government (if eligible under available AT grants) and self-funded individuals. However, the outcomes provide benefits and cost savings to interrelated government programs including healthcare, disability, and aged care. In this context, the government has a direct and indirect role in supporting smart home installations, stimulating better informed product choice and improving outcomes for the older population and people with a disability.

\subsubsection{Smart home AT costs}

Whether government supported or self-funded, the smart home market is characterised by rapidly growing ranges of increasingly integrated technologies. From an economic perspective, an overarching challenge, is to articulate core groups of product profiles and related subgroups of consumers that are likely to benefit. In our user survey and focus group discussions, the variation in products across a diverse range of housing settings is illustrated. As presented in previous sections, smart home AT is highly scalable where wireless and cloud-based platforms are established and can be routinely adapted with additional components to suit the needs of different consumers. As smart home technologies have shifted to generally wireless connectivity, products can also be more easily retrofitted, reducing costs and enabling easier use in rental accommodation. This starts to shape the elements of smart home AT costs which are comprised of upfront investment in infrastructure, and installation of integrated products and devices. 
In the area of dementia, a literature review examined AT support provided through formal government services as well as technology for private and personal use and concluding that both are important in policy concerned with more personalised services and care at home (Bowes et al. 2013). The review underlined the need for recent evidence of technology implementation given the rapidly evolving field, particularly in Information Communication Technology (ICT), electronic tracking devices and assistive devices in the home. The review also emphasised the range of potential technologies that may be integrated into the home including memory related (reminder systems), 'brain training' software and devices, mobility and tracking devices, healthcare monitoring, personal emergency alarm systems, home based monitoring and surveillance, remote peer support and remote informal caregiving.

Although a minority of studies reviewed included an economic analysis, one study indicated that living at home could be extended by an average of eight months, with an average residential care cost of $€ 3,000$. Thus, AT may deliver substantial savings in the cost of care over the long-term. There are also emerging technologies that can utilise a person's movement patterns; preliminary studies indicate that this data could be used to identify early signs of cognitive impairment. These new technologies could increase early detection and intervention and enable more effective management.

The Queensland Government undertook early research to inform smart AT service delivery models (Department of Communities Child Safety and Disability Services 2012). The 2012 report underlined the uncertainty of smart home AT adoption due to a lack of evidence in effectiveness, affordability and the rapid pace of technological progress. In the nine years since the report was published, the trend has continued with the development of new products, but without progression of any agreed or robust framework to align products with consumer needs. The key findings emphasised the positive client outcomes that smart home AT can provide in both aged care and disability settings. These positive outcomes include increased independence, confidence for living at home, feeling safer with general improvement in quality-of-life, and collectively avoiding or delaying the need for residential aged care. The report noted that risks of occasional technical malfunction were far outweighed by the benefits of the technologies for consumers. The disability and aged care assessment processes are thorough and are the control point to expand guidance on smart home options.

The Australian Productivity Commission has established the importance of trialling new ideas and removing outmoded regulation that prevents consumers and businesses accessing better services (Productivity Commission 2017b). The Commission acknowledges that digital technologies are challenging regulations and regulators, and policy changes are needed to manage new risks and facilitate new opportunities, but that ongoing slow response can pose a barrier to innovation and restrain investment in supporting infrastructure and cybersecurity (Productivity Commission 2017a).

\subsection{Policy development implications}

The global overview of current trends and policy frameworks provides a good foundation for a more accessible, flexible, and highly coordinated approach that puts the end-user at the centre of smart home AT solutions. A decentralised team approach in bringing together experts and stakeholders to hear and understand the end-users is key to the success of the Nordic model. This is especially important in smart home AT, where complexity is compounded by the intersection of care provisions and housing provisions. A clear articulation of who pays for what and who is responsible is paramount. The subsequent analytical chapters in this report will cover the policy implications from an end-user's perspective and provider perspective.

The benefits of smart home AT and its long-term impacts require additional research given its novel and emerging nature. However, the overall benefits of AT in increasing quality-of-life and prolonging independent living at affordable prices is internationally recognised. The Australian Government and funding bodies need to invest in quality evidence-based studies into off-the-shelf mainstream products to understand the requisite holistic provisions of both technological solutions and services. This also includes a responsibility in developing a system for better reviewing and managing the rapid global and national growth in loT and smart home AT devices entering into the market. The continued growth trend also means the Australian Government and funding bodies need to address the digital divide and increased inequalities created through inability to access technologies. 
A core finding is that there has been little progress in Australian Government implementation, service planning or funding guidelines for smart home AT. The commercial sector shows rapid growth anecdotally but is characterised by extensive product variations and limited guidance on product suitability and risks, as identified through the focus groups and in-depth case studies following. Additionally, there is very limited academic research published on smart home AT and related user experience, cost of products and related benefits. The review of the existing funding context in Australia in Chapter One shows that a more transparent and clear articulation on what can and cannot be funded under the aged care system and NDIS is paramount. There is an opportunity to draw on the Nordic model, in which clear communication and definition of responsibilities at different government levels benefits end-users in procuring AT that meets individualised aspirations and goals. The current NDIS assessment system and RAS planning has its flaws, particularly the lack of knowledge and training of planners and assessors. The Australian Government and funding bodies need to invest in expert knowledge and establish best practice frameworks for a rapidly moving smart home AT sector. This will help leverage the social and economic benefits of the technology. 


\section{User survey findings}

- There were 54 survey respondents; 15 provided complete and valid answers to the AQoL section of the survey, and 21 provided complete and valid answers to the QUEST section. While representative of the target population, the survey data is indicative rather than statistically generalisable. Nevertheless, it provides important insights.

- This is the first smart home AT survey conducted online targeted to people with disabilities or older persons.

- More younger adults responded to the survey (69\% versus $31 \%$ ), and there were slightly more men than women overall, reflecting perhaps more traditionally gendered roles in use of internet-based technology.

- Respondents reported a high level of disability-related impairment; over half self-identified as having between three and six functional limitations.

- Respondents were geographically diverse, but those living in remote areas (less reliable Internet, etc.) of less populous states like the Northern Territory (NT) were missing.

- Most respondents indicated that they self-funded their smart home AT purchases and received almost no government support.

- Most respondents were deploying smart home AT devices in many rooms within their home, with the living room being the most common area.

- Quality-of-life and utility evaluation survey technology (QUEST) results showed relatively high satisfaction with most smart home AT. Satisfaction was higher for Assistive Devices than for associated Services. 
- The uptake of smart home products reflects a characteristically chaotic emerging market segmentation with a correspondingly high variation in product ranges, specifications, functionality, quality and cost.

- Physical AT devices are the focus economically, but the expansion of cloudbased platforms that underpins low-cost integration of applications and devices to build virtual smart home environments but often been neglected.

- The value of smart home AT is implicitly driven by the ability to customise technology and design systems to meet individual needs. This supports a range of potential interrelated benefits across mental health, relationships and independence.

- The user survey included the Assessment of Quality-of-life (AQoL-4D) instrument. Preliminary results indicate consistent improvements across all dimensions including independent living, mental health, relationships, and senses. The initial positive Quality-of-life results, while based on a small sample $(n=15)$, are consistent with feedback reported through the focus groups and case studies. This suggests that smart home technologies support improved independence, activity, social and community connectedness and, as a result, potentially improved physical and mental health outcomes.

- Smart home AT may indirectly support a range of ongoing benefits, including extended independent living, smart home energy efficiency, safety and security, physical and mental activity, and healthcare monitoring.

There are five sections in this chapter. The first section reports on the process for the design and development of the survey questionnaire and the sampling methods. Section two outlines the demographics and describes the smart home AT used by our respondents. Section three presents a descriptive analysis of the Quality-of-life and utility evaluation survey technology (QUEST) results, while section four reports on the Assessment of Quality-oflife (AQoL-4D) results. Lastly, the economic analysis stemming from this data is detailed in section five. Sections one to four provide a primarily descriptive analysis of the respondents' home environments, their functional impairments, their smart home AT usage and reports on their life quality before and after their AT installation. Where a more structured, statistical analysis is possible, this is provided and combined with some discussion of the main findings of the survey, with a focus on the implications for government policy towards housing.

\subsection{Survey development}

Survey development, finalisation, sampling and analysis of specific elements were guided by best practice principles related to validity, reliability and gathering optimal response rates. Traditionally, AT survey responses have been solicited face-to-face, through the mail, or over the telephone and only more recently have online survey formats become standard (Forgasz et al. 2018). Further, all AT surveys of populations with functional difficulties to date have had very small sample sizes (typically less than 25 people) and vary widely in the types of AT evaluated from prosthetics to wheelchairs. 
No smart home AT survey of this type has ever been conducted online. Horevoorts et al. (2015) found no difference in response rates to paper versus online surveys and recommended forgoing paper surveys altogether. Previous researchers have noted that online surveys are viewed as the best means to facilitate access for people with disabilities as they are preferable in the case of individuals with diseases or conditions, including physical disabilities who are typically very difficult to reach (Wright 1999; Wright 2005; Corey et al. 2018). While prior research has shown that people with significant disabilities are hard to reach, it also indicated that). Thus, the research team assumed an online survey would be the most accessible for this type of research question as our targeted participants had a smart home ecosystem. However, a physical copy of the survey, or a modified version for those with particular needs, was available on request. Additionally, the survey could be completed by a family member or guardian if needed (Calveley 2012).

The Quebec User Evaluation of Satisfaction with Assistive Technology (QUEST 2.0), of which a modified version is embedded in the online survey instrument, has previously only been conducted in clinical settings where clinicians have direct access to clinical address databases (Larsson Ranada and Lidström 2019). Online surveys are much more cost-effective but are considered more problematic for the participation of those with chronic health conditions, functional impairments, and disability. Even with access to AT, severely disabled people are hampered in the use of online methods because of the perceived additional burden to complete tasks and the tight targeting of the content area (Solomon et al. 2017). Despite this known barrier, this research utilised an online survey because it is the best means of obtaining representative population samples and is the most convenient and contemporary method for reaching computer literate users (Hokke et al. 2018).

Given the smart home AT concept, we assumed that users would have high levels of internet access and usage. As such, an online survey would not pose an insurmountable challenge. However, online survey recruitment approval via human ethics panels relies on third-party data sharing policies and cooperation (Hokke et al. 2020). Thus, we sought support from 60 third-party organisations who had large databases most likely to reach our target audience and relied on them to distribute the survey on our behalf. For example, one of our third-party survey partners was NSW Seniors Card, which is designed to promote information and survey opportunities like ours to 1.4 million seniors. The project team undertook multiple rounds of follow up and survey reminders, and the timeframe was extended by several weeks to enable further responses. The final survey response varied for some content as individual questions were not mandatory, ranging from $n=30$ and a sample completing the $A Q \circ L$ of $n=15$.

The survey tool was developed following a systematic literature search. The search revealed previously used instruments with some reliability and validity, such as the Australian Quality-of-life (AQoL-4D) and Quebec User Evaluation of Satisfaction with assistive Technology (QUEST). However, no smart home AT survey tool previously existed, and only the AQoL component had Australian population comparison data. The QUEST was originally designed to measure the outcome of a single piece of AT, such as a wheelchair or switching system. Smart home ATs are an ecosystem comprised of several components. So, although the QUEST is the most commonly used tool for measuring AT outcomes, it has not been used for smart home ATs and has never been distributed in Australia previously. Therefore, the survey designed for this project included minor modifications to the QUEST to make it more appropriate to smart home AT systems. For instance, the reference to 'device' was altered to 'devices and an additional diagram was added to the beginning to ascertain what smart home ecosystem and components participants had. Please refer to Appendix One: User survey questions for more details.

The Assessment of Quality-of-life (AQoL), developed by Australian researchers, is a multi-attribute utility healthrelated quality-of-life instrument. While it can be used to measure health-related quality-of-life alone, its main purpose is to measure the 'utility' of health states (e.g. the preferences people have for different health states) in a way that is suitable for use in economic evaluation studies, particularly cost-utility analysis (Hawthorne et al. 1999). There are four AQoL instruments measuring various dimensions. In this survey, we used the AQoL-4D, which measures Independent Living, Mental Health, Relationships, and Sense. The AQoL-4D was selected because it was the shortest version and the dimensions measured were the most relevant to smart home ATs as identified in the literature (lenca et al. 2017; Khosravi and Ghapanchi 2016).. It is also the In the before and after modification previously conducted, the developers from the Centre for Health Economics approved the final survey design (Carnemolla and Bridge 2016b). This modification was piloted successfully to measure the 
quality-of-life before and after housing change (Carnemolla and Bridge 2016b; 2019). The modified version of the AQoL-4D used in our survey determined the quality-of-life before and after smart home AT installation. The before and after modification to the AQoL-4D enabled an assessment of the utility scores, which could then be compared to existing Australian population norms (Hawthorne et al. 2013) derived from the 2007 Australian user survey of mental health and wellbeing as comparators.

The final section of the survey contained demographic questions to ascertain age group, gender, income bracket, housing tenure, living arrangements, and source of income. Only three age brackets were provided: 17 and under, 18 to 64 , or 65 plus. At the end of the demographic section, participants were invited to provide their contact details if they were willing to be contacted to elaborate on their survey answers. We used this data to recruit participants for the case study vignettes.

The user survey was designed to be completed online via the most prevalent survey platform: Qualtrics (Carpenter et al. 2018). Used by 2,000 universities, Qualtrics offers web-based and mobile-friendly versions of surveys, allowing participants to complete the survey on a tablet or mobile phone. In addition, Qualtrics has an embedded accessibility assessment tool that diagnoses the survey in compliance with the Web Content Accessibility Guide (WCAG) 2.0 AA standards. Participants were able to navigate through the survey at their own pace, saving and returning to the survey later if needed.

It is well known that people with significant disabilities are hard to reach. Online surveys facilitate access to a population that is difficult to reach (Wright 2005). It is also important to note that whilst people with cognitive disabilities were not the sole focus of this research, they are often beneficiaries of smart home AT; automation can be deployed for safety, to remove environmental complexity and for reminding. However, people with cognitive disabilities are often implicitly or explicitly excluded from the research of this type, in part because they may be perceived as lacking capacity to provide informed consent. However, as Horner-Johnson and Bailey (2013) note, including people with severe and profound cognitive disabilities is empowering because the research can make important findings about their experience of self-care, which has implications for improved practice and policies.

\subsection{Survey methods and sampling}

The survey was designed and validated on highly regarded Qualtrics survey development and analysis platform (Ginn 2018). The Qualtrics platform also has the option to include graphics, which we used, so that survey respondents could upload photos of their in-home smart home AT ecosystem. The development and refinement of the draft took place over several iterations and involved detailed feedback from all researchers as the four sections were developed and modified for face validity. Additionally, Qualtrics' internal validation engine (ExpertReview) was used to validate the overall quality, and confirm there were no internal survey logic errors, the survey was fully accessible, and there was no potential for response fraud. Unfortunately, despite feedback from our case participants that 'completion of the survey was easy', the duration and number of free text boxes was greater than that recommended by ExpertReview (i.e., surveys longer than nine minutes start to see substantial levels of respondent break-off when using mobile devices).

The survey could not be launched until clearance from the Human Research Ethics Committee at the University of NSW (HC190361) was received. This took longer than anticipated due to the inter-state collaboration and the need for ethics approval to be ratified by the Universities of South Australia and Tasmania respectively. Importantly, the national statement that is enforced by the Human Research Ethics Committee, precludes any direct recruitment of research participants to protect vulnerable respondents. As such, the survey was distributed solely by third parties. Biernacki and Waldorf (1981) recommend snowball sampling via third-party distribution for sub-group within the wider social networks of specialised disability and older people's groups serviced by Non-Government Organisations (NGOs) and aligned well with AT housing providers and end-users NGO engagement in other parts of our research strategy. 
Human ethics committees exist to protect vulnerable individuals, especially where the potential for cognitive impairment exists and the ethical clearances provided specified using an authorised third-party as an established means to prevent being provided with lists of individuals who had smart home AT to approach directly (Becker et al. 2004). Consequently, we had to rely on 'gatekeepers' or third-party organisations who had direct access to our target population. We had to publicise the study through programs that provided services to such individuals or groups that advocated for them. However, as Becker et al. (2004) note, it is common in larger NGOs to request already overburdened staff members to undertake this communication via social media, newsletters and other scheduled media and this can impact response rates and survey engagement adversely.

In line with our ethics clearance, the survey was designed to explore and measure the impact of smart home AT system pros and cons and was distributed as widely as practicable. Sixty NGOs were identified using the extensive network of the research team, who are leading experts in the field. As a result, 20 third-party organisations agreed to promote the survey to their members. Please see Appendix Two for a list of all the organisations that distributed information about the survey. We also attempted to increase awareness of the online survey via the Home Modification Clearinghouse social media accounts, including Linkedln (500+), Twitter (217) and Facebook (72). Social media supplementation has been recognised as important because of its cost-effectiveness and potential for greater spread (Forgasz et al. 2018).

The survey flyer was distributed digitally, apart from one physical flyer pinned to the noticeboard in one accommodation site. A total of 20 organisations agreed to circulate the survey flyer via their respective communication channels, ranging from their social media outlets to physical flyers in their accommodation sites. In addition, the user survey was also promoted on the Home Modification Clearinghouse (https://www.homemods.info/) website through a news slide on the home page and associated social media channels. After the initial distribution, participants in the focus groups were also asked to distribute the survey in their respective organisations.

Completion of the survey was expected to take approximately 15 minutes and analysis of completion times indicated that the median completion time was 11 minutes. Of the 54 survey attempts, 21 (39\%) completed enough of the survey for the QUEST section to be analysed, whilst even less completed the AQoL section i.e., $15^{1}$ (28\%). The survey was open from the beginning of May to the end of December 2019, with the first survey response on 1 May 2019 and the last response 27 November 2019.

\subsection{User survey findings}

The user survey had five sections (see Appendix One). This included:

- Demographics, AT funding sources and functional impairment(s)

- Smart home AT used by respondents

- Quebec user evaluation of satisfaction

- Quality-of-life and AT

- Option to provide more detail and greater context of their AT smart home purchase via individual case studies.

\subsubsection{Survey demographics}

This section provides a context for understanding the survey responses regarding the quality-of-life, AT satisfaction and AT components. The enrolment in the online survey was lower than expected. This may be a consequence of several factors. First, recruiting people who are older or who have significant difficulties presents a variety of challenges, due largely to the burdens of living with coexistent illnesses (Brostow et al. 2015). Secondly, to meet

1 The AQoL survey sample included two responses which had missing data for a few of the total 12 questions. In these cases the missing questions were assumed to be constant before and after to provide neutral responses for these questions with no incremental change. 
our tightly targeted impairment specific AT users is known to pose a greater the degree of difficulty, so the online survey had to be easy to understand, disability friendly and appropriately responsive to changes in functional performances and quality-of-life with anyone who has health conditions or impairments, (Kadam et al. 2016).

The demographic questions were the last part of the user survey, and so had the lowest number of usable responses. Of the 21 usable responses, there were none from respondents under 18 years and eight (38\%) respondents did not state either their gender or their age. Thus, the achieved survey sample for age and gender demographic data included only a population of 13 adults (62\%) with moderate to severe functional limitations aged 18 plus. As can be seen in Table 5, there was a higher percentage of younger adults (aged 18-65) and slightly more men than women amongst the respondents. More of the older respondents were male, reflecting perhaps more traditionally gendered roles, whilst the reverse is true for the younger adults. This is expected as, whilst there is growing evidence of the benefits of smart home AT for older adults, adoption rates are typically lower compared with younger adults (Mitzner et al. 2019), as are gendered roles.

Table 5: What is your age group?

\begin{tabular}{|c|c|c|c|c|c|}
\hline \multicolumn{2}{|c|}{ What is your age group? } & \multirow{2}{*}{$\begin{array}{r}\text { Male } \\
4\end{array}$} & \multirow{2}{*}{$\begin{array}{r}\text { Female } \\
5\end{array}$} & \multirow{2}{*}{$\begin{array}{r}\text { Count } \\
9\end{array}$} & \multirow{2}{*}{$\begin{array}{r}\text { Percentage } \\
69 \%\end{array}$} \\
\hline $18-65$ & Count & & & & \\
\hline & $\%$ of Total & $44 \%$ & $56 \%$ & & $100 \%$ \\
\hline \multirow[t]{2}{*}{ Above 65 years } & Count & 3 & 1 & 4 & $31 \%$ \\
\hline & $\%$ of Total & $75 \%$ & $25 \%$ & & $100 \%$ \\
\hline Totals & & 7 & 6 & 13 & $100 \%$ \\
\hline
\end{tabular}

Missing: 8.

Source: Authors.

Geolocation data records the location of the respondent at the time of completing the survey. Respondents could provide their geolocation data in the form of latitude and longitude automatically through their web browser. However, for privacy reasons, the user was asked for permission to report location information and most chose to block this. In two cases, the respondent gave a different state as their primary residence, i.e., the geolocation is recorded as Tasmania, but the state of residence in Victoria. As shown in Table 6, of the provided geolocation data, a national response is evident, but there was no record of participation from the Northern Territory (NT) and it is likely that there were less survey respondents from remote areas (less reliable Internet, etc.) and from the less populous states like the NT. For instance, Mitzner et al. (2019: 17) states that, 'Despite rapid digital development in the past two decades, the remote parts of Australia still experience disadvantages with the adoption and diffusion of digital technology'.

Table 6: Geolocation by state or territory and gender

\begin{tabular}{lrrr}
\hline Geolocated respondents & Male & Female & Count Percentage \\
\hline NSW & 3 & 3 & 6 \\
\hline SA & 1 & 0 & 1 \\
\hline QLD & 1 & 0 & 0 \\
\hline TaS & 1 & $9 \%$ \\
\hline ACT & 9 & 1 & $9 \%$ \\
\hline WA & 1 & 0 & 1 \\
\hline Total & $\mathbf{1 1}$ & $\mathbf{1 0 0 \%}$ \\
\hline
\end{tabular}

Missing: 10 .

Source: Authors. 
All respondent's main language was English, and most were born in Australia except for two respondents, one of whom was born in New Zealand and the other in Canada. The absence of non-English speaking respondents is unsurprising, as being non-English speaking combined with lack of acculturation to provision of smart home AT are known issues associated with greater difficulty reaching these groups (Bethlehem 2010). Prior research has associated these same issues with poorer outcomes and lack of access to AT service delivery (Parette et al. 2004).

As can be seen in Table 7, nearly 60 per cent of respondents had never been married or are separated. This is also unsurprising; living alone makes smart home AT more attractive. This is because AT has the potential to provide greater autonomy and substitute both informal and paid care. Prior research notes that home modification, such as smart home AT, can directly substitute reliance on both formal and informal care (Carnemolla and Bridge 2019).

Table 7: Martial status of respondents

\begin{tabular}{lrr}
\hline Marital status & \\
\hline Never married & 5 & $41.7 \%$ \\
\hline Married & 3 & $25.0 \%$ \\
\hline Separated but not divorced & 2 & $16.7 \%$ \\
\hline Widowed & 1 & $8.3 \%$ \\
\hline Divorced & 1 & $8.3 \%$ \\
\hline Total & 12 & $100.0 \%$ \\
\hline
\end{tabular}

Source: Authors.

\subsubsection{Understanding respondent's residential accommodation}

Having a clearer understanding of the respondent's residential accommodation raises issues about communication with landlords and body corporate committees about fixed installations such as smart openings, and automatic service shutoffs, which are critical for those with cognitive and mobility impairments. While just over a third of respondents were buying or outright owners (as expected amongst older people), the majority or two-thirds were in less secure tenure. Interestingly, as shown in Table Eight: Primary accommodation of respondents, this finding aligns with the Australian Institute of Health and Welfare (AlHW) 'People with disability in Australia' web report (2019) that found while more respondents under the age of 65 with a disability (64\%) own their home, there are a significant proportion who reside in social housing. The AlHW noted that Specialist Disability Accommodation (SDA) accounted for approximately 16 per cent and another 20 per cent rented from a social housing provider (Australian Institute of Health and Welfare 2019). In our survey, 50 per cent ( $n=3$ ) of those respondents who rented had social housing landlords. The other 50 per cent $(n=3)$ were in community housing $(17 \%, n=1)$ or renting in the private market $(33 \%, n=2)$.

Table 8: Primary accommodation of respondents

\begin{tabular}{lrr}
\hline Current accommodation & & \\
\hline Rental - Private Market or Social Housing & 6 & $50.0 \%$ \\
\hline Owner - Outright or Mortgaged & 4 & $33.3 \%$ \\
\hline With Family or Relatives & 1 & $8.3 \%$ \\
\hline Specialist Disability Accommodation & 1 & $8.3 \%$ \\
\hline Total & 12 & $\mathbf{1 0 0 . 0 \%}$ \\
\hline
\end{tabular}

Source: Authors. 
As can be seen in Table 9, the type of residential accommodation in which our respondents reside ranges from apartments to stand-alone dwellings. Dwelling type is important to the deployment of smart home AT; it impacts the requirements under residential housing guidance such as the SDA and 'Livable Housing' guidance documents. It also determines who needs to provide authority to carry out works involving structures.

The Australian Building Codes Board, under the Accessible Housing project, is currently reviewing regulatory analysis to determine potential minimum accessibility standards for housing applied through the National Construction Code (NCC). Under the NCC, residential accommodation for all Australians is divided into classes. The NCC building classes determine mandatory accessibility features. Only Class Three, and common areas within Class Two housing (i.e. any building containing two or more sole-occupancy units each being a separate dwelling) currently requiring access and mobility standards for design and modifications. The NCC and Australian Standards have historically not included smart home AT. Although, many new SDA accommodation providers and aged care providers have noted this as a marketable difference for improved quality-of-life (see focus group discussion in chapter four for more details). The Accessible Housing project is currently considering the Liveable Housing Design Guidelines and additional options identified through consultation. It will be important to note if smart home AT is mentioned or considered.

Table 9: Percentage of respondents by type of residential accommodation

\begin{tabular}{lrr}
\hline Type of accommodation & \\
\hline Separate house & 5 & $41.7 \%$ \\
\hline Flat, unit or apartment & 5 & $41.7 \%$ \\
\hline Semi-detached, row or terrace house, townhouse & 1 & $8.3 \%$ \\
\hline Other dwelling & 1 & $8.3 \%$ \\
\hline Total & 12 & $100.0 \%$
\end{tabular}

Source: Authors.

Table 10: Length of residency in current accommodation, shows that just under 60 per cent of respondents have lived in the same dwelling for more than five years. One person had lived in their current home for more than 20 years. This finding is consistent with the home modification literature which states that, for many older people, living in your home for longer periods is a factor in adaptation uptake (Keglovits and Stark 2020). Many older people are reluctant to make significant and expensive changes to their residential accommodation the older they get because increased needs can mean transitioning your private residential space to a more public arena (Jarling et al. 2018).

Table 10: Length of residency in current accommodation

\begin{tabular}{lrr}
\hline Time in current accommodation & & \\
\hline Under 1 year & 1 & $8.3 \%$ \\
\hline $1-4$ years & 4 & $33.3 \%$ \\
\hline $5-9$ years & 4 & $33.3 \%$ \\
\hline $10-20$ years & 2 & $16.7 \%$ \\
\hline Over 20 years & 1 & $8.3 \%$ \\
\hline Total & 12 & $100.0 \%$
\end{tabular}

Source: Authors. 
Respondents reported a high degree of functional limitations, with a range of two to seven (out of 12). Many respondents also reported a high level of co-morbidity with over half self-identifying as having between three and six functional limitations each. As can be seen in Figure 3, the greatest number of respondents had a mobility impairment affecting the coordination of their hands or legs, followed by impairments of hands and feet, underscoring the need for voice command. Several respondents also self-identified with cognitive loss and extremes of size and weight.

Figure 3: Respondents who identified one or more functional impairments

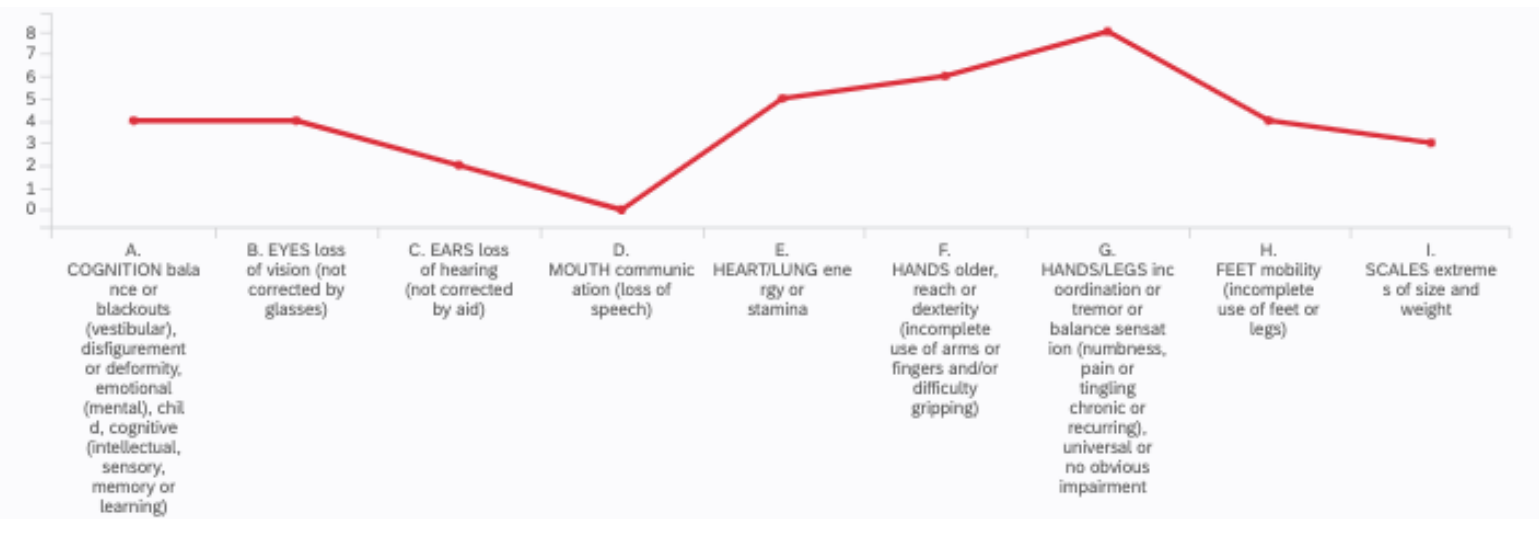

Source: Authors

\subsubsection{AT funding sources}

As presented in sections two and five, the potential for government funding is available across disability and aged care settings. Most of our respondents $(83 \%, n=25)$ indicated that they are self-funded and had received no government support. Of the 17 per cent $(n=5)$ who did receive some level of government assistance (integrated with self-funded components), 40 per cent $(n=2)$ indicated that this was from a disability pension, while 60 per cent $(n=3)$ identified as having received some NDIS funding to purchase their smart home AT.

\subsubsection{AT used by respondents}

Survey respondents used the full gamut of smart home AT. Respondent's smart home AT ranged from a complex integrated set-up designed to maximise independent living for those with significant impairments to a much simpler camera or telehealth carer set-up for monitoring older people's safety. Components which are typically part of a complex integrated system smart home system include command systems such as Google Home, Amazon Alexa, Apple HomePod, Microsoft Cortana and others. In our survey $(n=25)$ respondents who indicated their system types were as follows: Google Home 38 per cent; Amazon Alexa 13 per cent; Apple HomePod 6 per cent; Microsoft Cortana 9 per cent; and other 34 per cent where the specific device(s) remained unidentified. Given that 47 systems were noted, many respondents have multiple systems and sometimes one in each room of their dwelling. Unsurprisingly, Google Home is the most common voice controlled personal assistant in use amongst respondents; it has been available for the longest time (being released in 2016 and the mini released in 2017). Voice control systems are typically used to control smart home appliances. Respondents had a range of interface devices ( $n=70$ ) connected to these systems, including smart speaker(s) 21 per cent; tablet interface 23 per cent; smart phone interface 36 per cent; and other 20 per cent (again textual responses to other were unclear with one person mentioning smart lights indicating they may not have fully comprehended the question). Smart phones were the most popular, presumably because of portability and familiarity. 
The smart home AT devices that our respondents stated that they had acquired ranged across five categories as follows:

- Entertainment ( $n=26)$ : smart TV (53\%), smart audio systems (15\%), white noise control (4\%), automated lighting (27\%)

- Thermal ( $n=23)$ : automated air conditioning (35\%), automated fan (23\%)

- Safety ( $n=21)$ : security camera (19\%), alerting reminder system(s) (19\%), gas automatic shutoff (5\%), electricity automatic shutoff (10\%), water main automatic shutoff (5\%), smart smoke detectors (43\%)

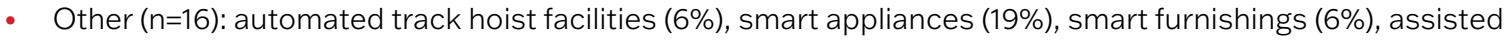
breathing device (13\%), home backup generator or battery (13\%)

- Openings ( $n=11)$ : automated curtain (3\%), blind, window (3\%) or door controls (5\%).

Figure 4 illustrates that nearly all respondents were using their smart home AT for entertainment, which is unsurprising given that this is how the majority are commercially marketed. However, two-thirds were using their smart home AT for safety and thermal comfort and just over one-third was also using it for operation of openings in the home. This is consistent with our functional impairment reporting and with solutions that enable greater autonomy where severe restrictions of mobility exist.

Figure 4: Percentage of respondents by the type of smart home AT in use

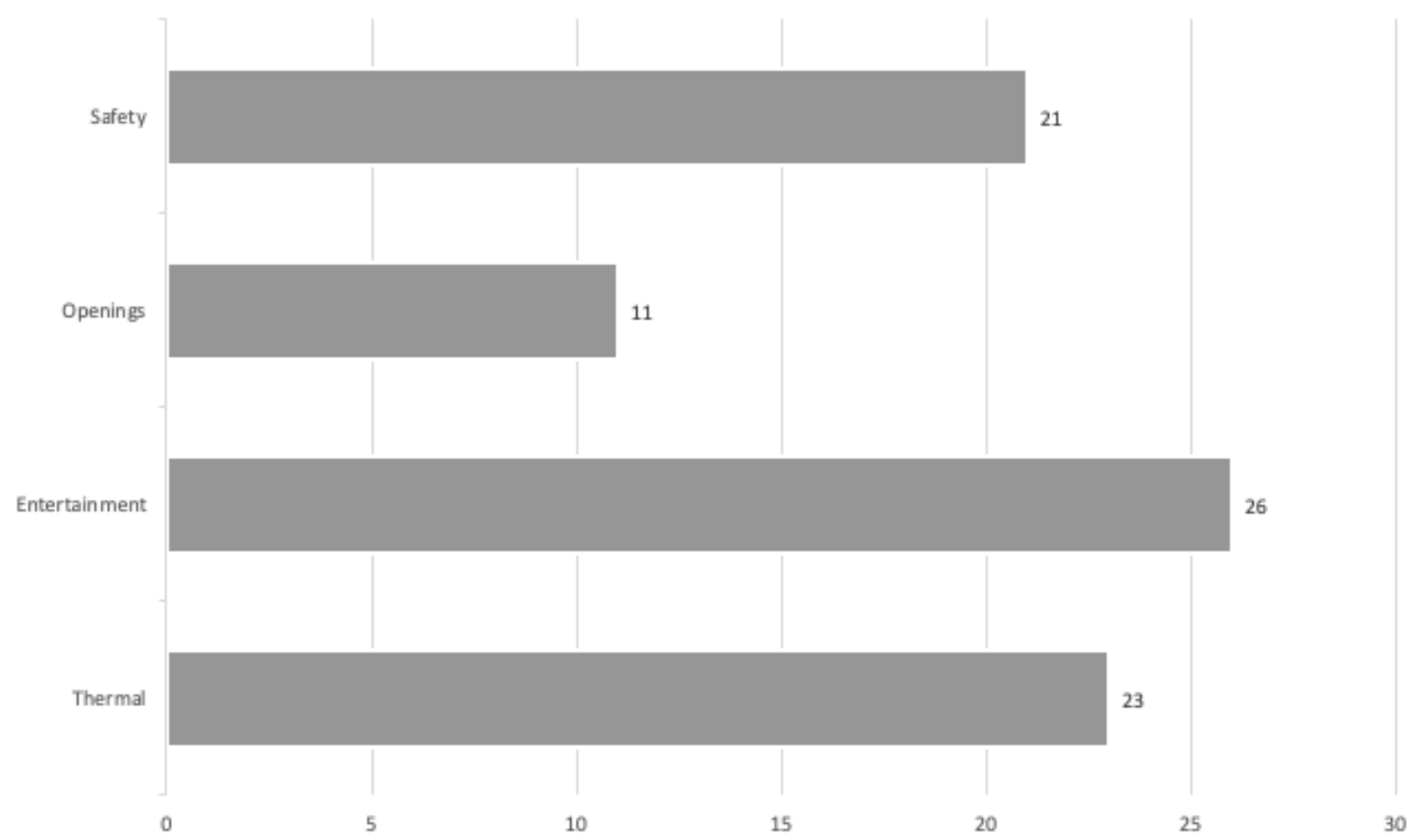

Source: Authors.

Respondents identified many rooms $(n=56)$ within their home, and areas outside their home, where they had installed smart home AT devices. The living room (20\%) was the most commonly cited room, which is unsurprising as entertainment was the most commonly cited activity. The garage or gate was the least commonly cited place of installation at 5 per cent. Again, this finding is expected given devices for openings was relatively low and many respondents may not drive due to the severity of their functional impairments, age or a combination of both. 
Figure 5: Rooms in the home where that the smart home AT system is deployed

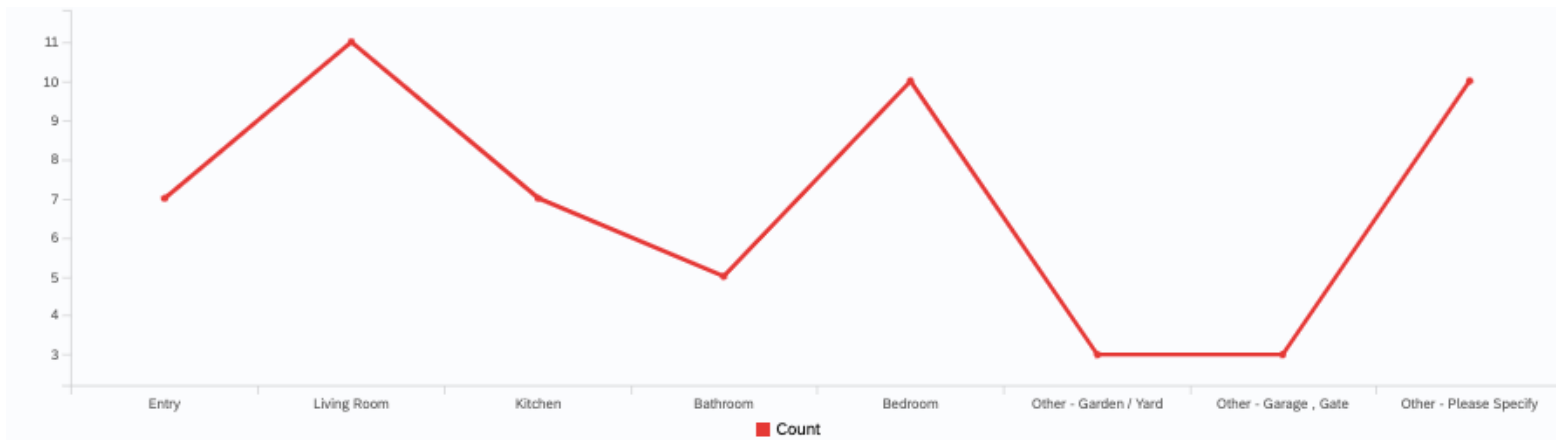

Source: Authors.

\subsubsection{User evaluation of smart home AT}

This section reports the survey findings based on the Quebec User Evaluation of Satisfaction with assistive Technology or QUEST (Demers et al. 2002), which was designed to measure the outcome of AT solutions. This standardised survey is the most widely used instrument for measurement of AT satisfaction from a user perspective and it covers both Devices and Services. Developed in response to the need for outcome measurements for rehabilitation and AT prescription, QUEST was the brainchild of Demers et al. (2002) who were inspired by a model of satisfaction developed in the late 1990s (Simon and Patrick 1997). Despite QUEST being the most widely utilised standardised survey tool, a previously identified issue regarding ratings of multiple devices and items relevance was noted. This issue was that the weight of any device may be relevant to portability or prosthetics but is problematic and potentially confusing for rating satisfaction of a smart home AT system (Goodacre and Turner 2005). So, in line with previous work, minor modifications were made to clarify this language to try to reduce non-response or inapplicable survey responses. For example, Wessels and Witte (2003) previously noted that minor changes reduced the amount of missing data when the QUEST was used with a range of ATs because respondents were better able to answer questions that might otherwise have been skipped. It is also important to note that while limitations are associated with one timepoint only, responses for comparison of before and after impacts of smart home AT is proven as a useful analysis technique (Carnemolla and Bridge 2016b; Carnemolla and Bridge 2019). Despite limitations, the survey analysis revealed that the respondents investing in smart home AT had a positive qualityof-life and satisfaction outcome impact.

Table 11 indicates that the lowest mean in our dataset was $3.2(S D=1.19)$ for Services and the highest mean was 3.50 (SD =1.19) for Devices. This means that, overall, respondents reported a greater level of satisfaction with Devices compared to Services. It is also important to understand where and how much variation is represented within our sample, and an examination of the mean value for each scale plus or minus one standard deviation shows how tightly clustered scores are. In a normal distribution, approximately 68 per cent of the scores fall within the range of the mean, plus or minus one standard deviation. For the Devices sub-scale one can note that the scores within one standard deviation of the mean have a narrower range (2.31 to 4.69) and the scores fall closer to the higher range of possible values in the range from 1.00 to 5.00 (with a minimal score of 3.24 and a maximum of 3.87). However, the range of scores within one standard deviation of the mean for the Services sub-scale is wider (3.56 to 4.56), even though the minimum score is the same as that for Devices. 
Table 11: Descriptive statistics for the QUEST 2.0 from the smart home AT Survey $(n=20)$

\begin{tabular}{lrrrrrr}
\hline Subscale & M & SD & SCORES WITHIN & M \pm 1 SD & MAX & RANGE \\
\hline Device & 3.50 & 1.19 & 2.31 to 4.69 & 3.24 & 3.87 & 0.63 \\
\hline Services & 3.2 & 1.36 & 1.84 to 4.56 & 3.24 & 3.62 & 0.38 \\
\hline Total & 3.47 & 1.26 & 2.2 to 4.73 & 3.24 & 3.86 & 0.62 \\
\hline
\end{tabular}

Source: Authors.

Item by item analysis

As indicated in Table 12, the results of the QUEST indicated overall relatively high satisfaction with most smart home AT acquired by our respondents. Figure 6 illustrates the ranked total satisfaction scores, where total satisfaction is an aggregation of our respondents' top three responses (i.e., satisfied, quite satisfied and very satisfied). As can be seen in Table 12 and Figure 6, the highest satisfaction scores were reported for comfort, which rated 95 per cent; ease of use, which rated 91 per cent; and effectiveness to meet their needs, which rated 81 per cent. The lowest satisfaction related to after sales items of follow up service and professional service.

Figure 6: Overall satisfaction of AT by category

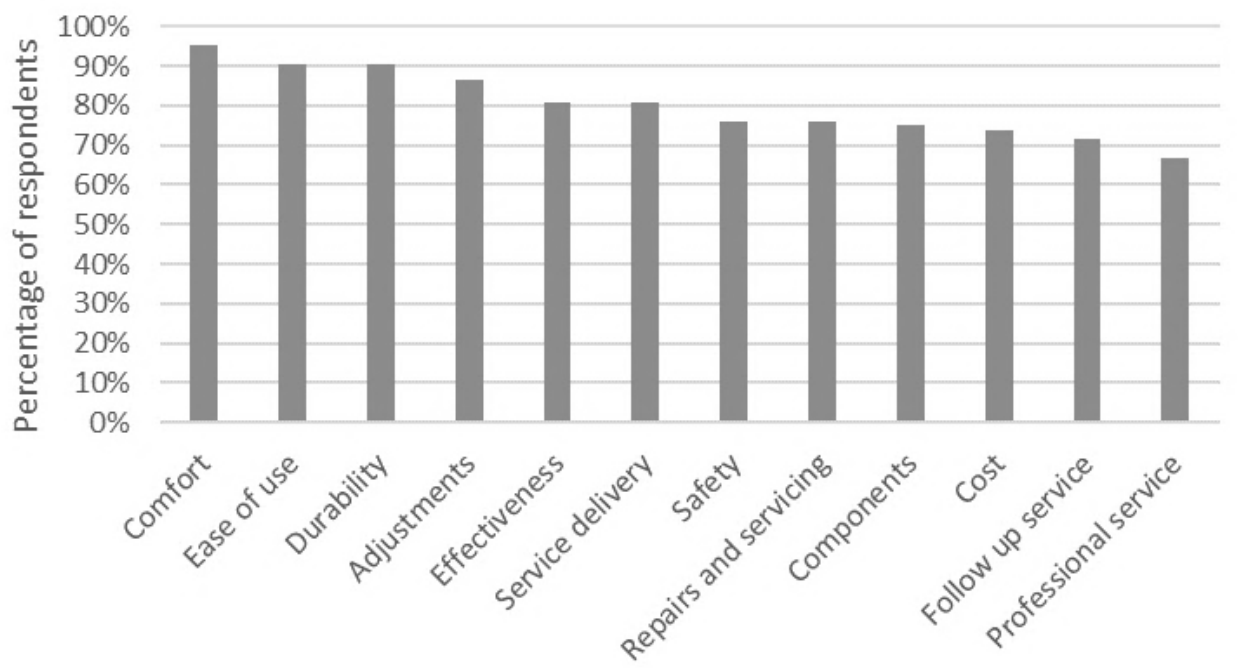

Satisfaction category

Source: AT user survey ( $n=20$ to 24 across respective questions).

Three-quarters of respondents indicated satisfaction with safety and repairs, but this and other service categories were notably lower than their satisfaction with the comfort or ease of use that the devices provided. Service satisfaction at 77 per cent for their smart home AT is reasonably high but less than most services would aspire to. Our respondents rated their overall satisfaction with their smart home AT components as being approximately two-thirds satisfied (75\%) overall because of factors such as less than desired follow up and lack of professional services in some respondents' experience. 
Table 12: Item by item analysis for the Device sub-scale for smart home AT in the home $(n=21)$

\begin{tabular}{lrrr}
\hline Items & $\begin{array}{r}\text { of respondents } \\
\text { "more or less } \\
\text { satisfied" }\end{array}$ & $\begin{array}{r}\text { of respondents } \\
\text { "quite or very } \\
\text { satisfied" }\end{array}$ & $\begin{array}{r}\text { Total Satisfaction } \\
\text { ratings }\end{array}$ \\
\hline Comfort & $33 \%$ & $62 \%$ & $95 \%$ \\
\hline Ease of use & $24 \%$ & $67 \%$ & $91 \%$ \\
\hline Service delivery & $24 \%$ & $58 \%$ & $82 \%$ \\
\hline Effectiveness & $29 \%$ & $52 \%$ & $81 \%$ \\
\hline Safety & $29 \%$ & $48 \%$ & $77 \%$ \\
\hline Repairs and servicing & $29 \%$ & $48 \%$ & $77 \%$ \\
\hline Components & $21 \%$ & $54 \%$ & $75 \%$ \\
\hline Cost & $22 \%$ & $52 \%$ & $74 \%$ \\
\hline Follow up service & $29 \%$ & $43 \%$ & $72 \%$ \\
\hline Professional service & $24 \%$ & $43 \%$ & $67 \%$ \\
\hline
\end{tabular}

Source: Authors.

Table 12 compares the more nuanced unaggregated satisfaction results. It shows that respondents ranking order reflecting what they were most satisfied with as distinct from what they were least satisfied with. As can be seen, satisfaction with professional service was the lowest satisfaction score overall at 67 per cent, meaning that just under a third were unsatisfied with this aspect of their smart home AT experience. From their perspective importance ease of use was the most critical whilst follow-up service was the least important. Whilst comfort and follow-up service are in the listed as 5 per cent as Figure 7 shows in terms importance there is clearly a relationship to ease of use and effectiveness, and it is unsurprising that respondents are looking for an out of box or effectiveness satisfaction that is highly effective.

Figure 7: Most satisfied assistive technology

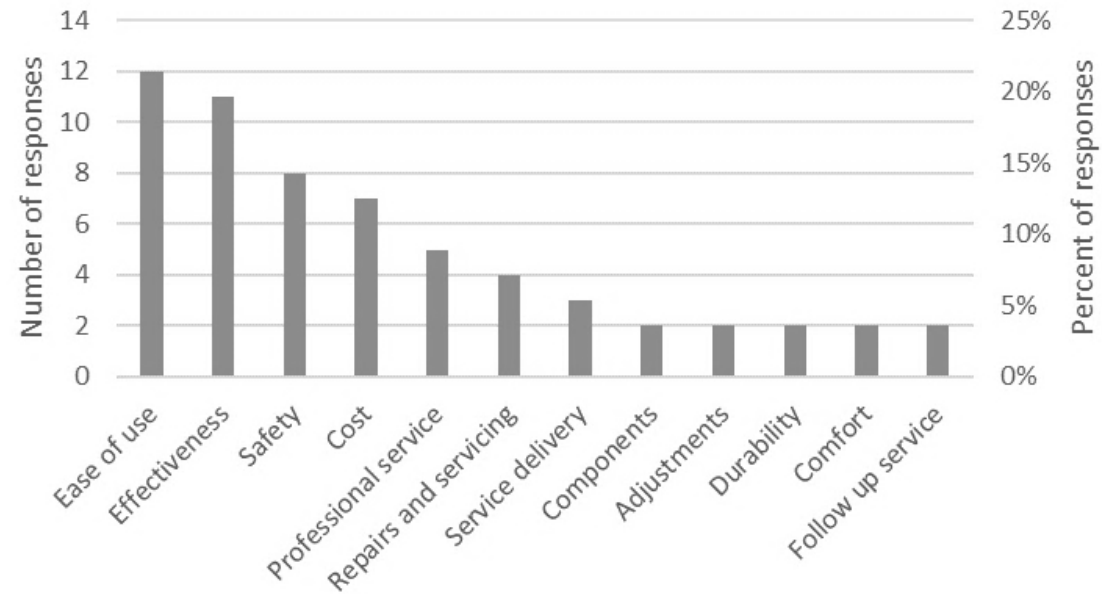

Satisfaction category

Source: AT user survey ( $n=20$, compiled from respondents reporting the 3 most important items).

Overall, respondents reported satisfaction as generally high across all satisfaction categories with half of all satisfaction questions scoring 77 per cent or higher. On the other hand, lower QUEST scores indicate respondents experienced critical problems and corrective actions are required in order to increase the effectiveness of assistive technologies. 


\subsubsection{Quality=of-life and AT}

The survey data included items on care hours and the 12 question Assessment of Quality-of-life (AQoL-4D) instrument to estimate health-related quality-of-life utility scores. The AQoL-4D was included in the survey as a short 12 multiple choice questionnaire covering four AT relevant dimensions of independent living, mental health, relationships, and senses (Hawthorne et al. 1999). The AQoL-4D instrument estimates utility using a three-stage procedure to produce the final estimate of a health state utility (Richardson, Peacock, lezzi, Day, \& Hawthorne, 2007). Figure 8 shows us that most respondents were independent with basic Activities of Daily Living $(n=8)$ prior to their smart home AT purchase, but just under half had issues with dressing, bathing and so on $(n=7)$. Whilst the need for assistance with the most difficult task remained, the number who needed daily assistance reduced after the AT smart home purchase.

Figure 8: Change in Activities of Daily Living (ADL) status using smart home AT (eg. bathing, dressing, etc.)

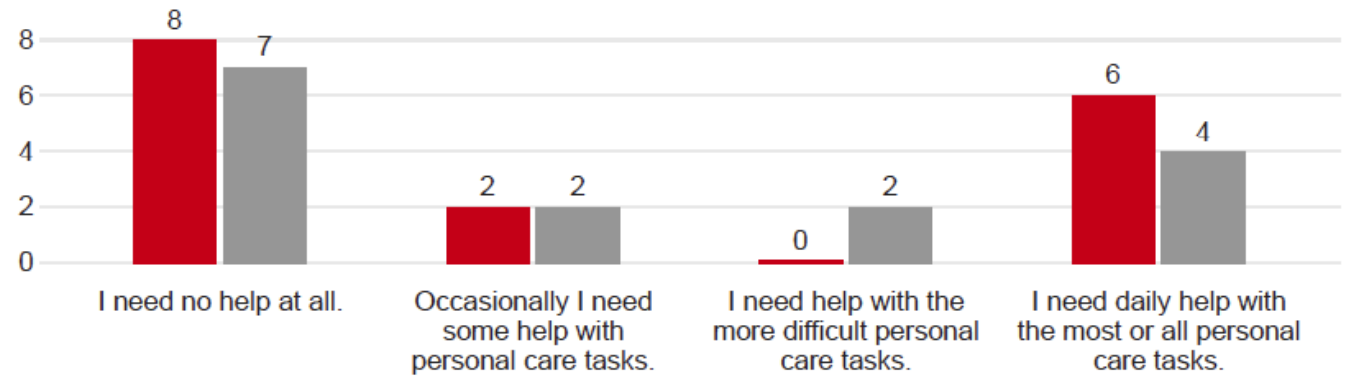

BEFORE use of smart home assistive device(s) AFTER use of smart home assistive device(s)

Source: Authors.

Figure 9 shows that the smart home AT was most significant in the reduction of the need for assistance with daily tasks. The majority of respondents indicated that after their smart home AT was installed, they did not need any help with cooking, cleaning and so on. This reduction in the need for assistance was true even for the most difficult household tasks.

Figure 9: Change in Intrinsic Activities of Daily Living (IADL) status using smart home AT (e.g. cooking, house cleaning, etc.)

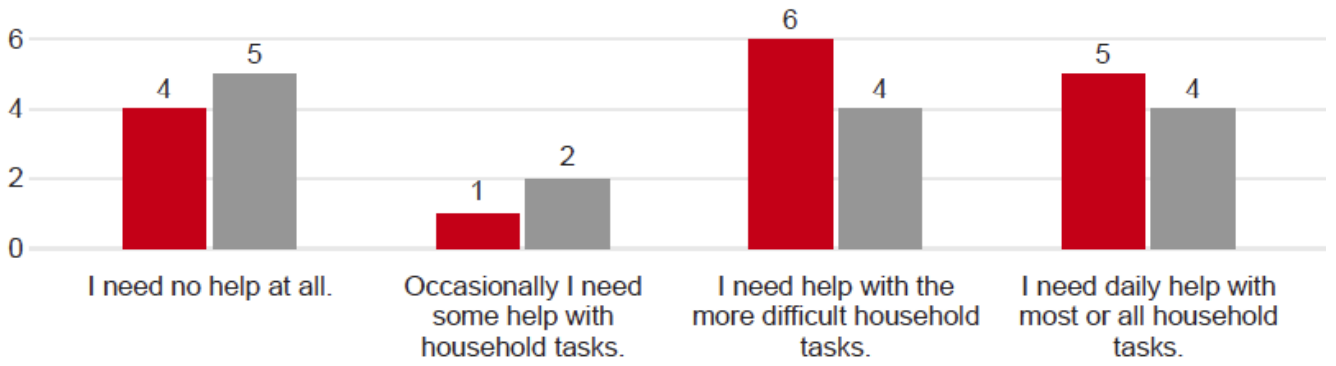

BEFORE use of smart home assistive device(s) AFTER use of smart home assistive device(s)

Source: Authors.

As expected, smart home AT purchased by respondents had little to no impact on mobility inside or outside the home as most of this technology remains within the home, and almost no respondents indicated purchase of automated track hoists or the like. Interestingly, 50 per cent of the respondents indicated that it was difficult to get around their home and community, or they could not do so at all, and this remained unchanged. Figure 10 illustrates that for some participants, greater use of smart home AT reduced the quality of their relationships. However, most respondents noted that after the use of their smart home AT there were sometimes greater instances of close and warm relations $(n=9)$. 
Figure 10: Change in perceived quality of relationships

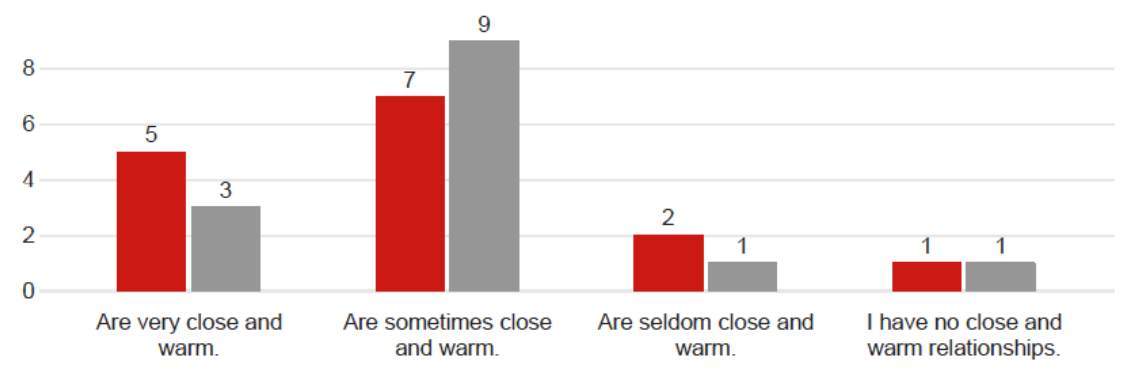

- BEFORE use of smart home assistive device(s) AFTER use of smart home assistive device(s)

Source: Authors.

Figure 11 illustrates the reduction in social isolation afforded by the smart home AT systems for several respondents. Almost half of respondents noted a reduction in social isolation after their AT system purchase and more respondents indicated that they had more friends. Presumably, this is because the smart systems facilitate online social participation. Plus, the high number of respondents using their AT systems for entertainment provides opportunities for activities that are valued but difficult without smart home AT, such as independently listening to music.

Figure 11: Change in perceptions of social isolation

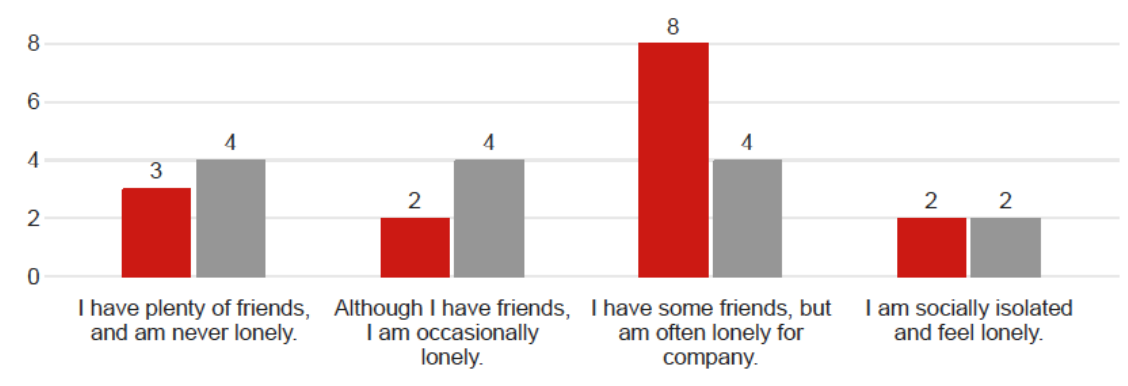

- BEFORE use of smart home assistive device(s) $\quad$ AFTER use of smart home assistive device(s)

Source: Authors.

Figure 12 focuses on the value of the smart home AT for visual activities. A significant number of respondents noted an improved ability to focus and read small print.

Figure 12: Perceived change with visual activities

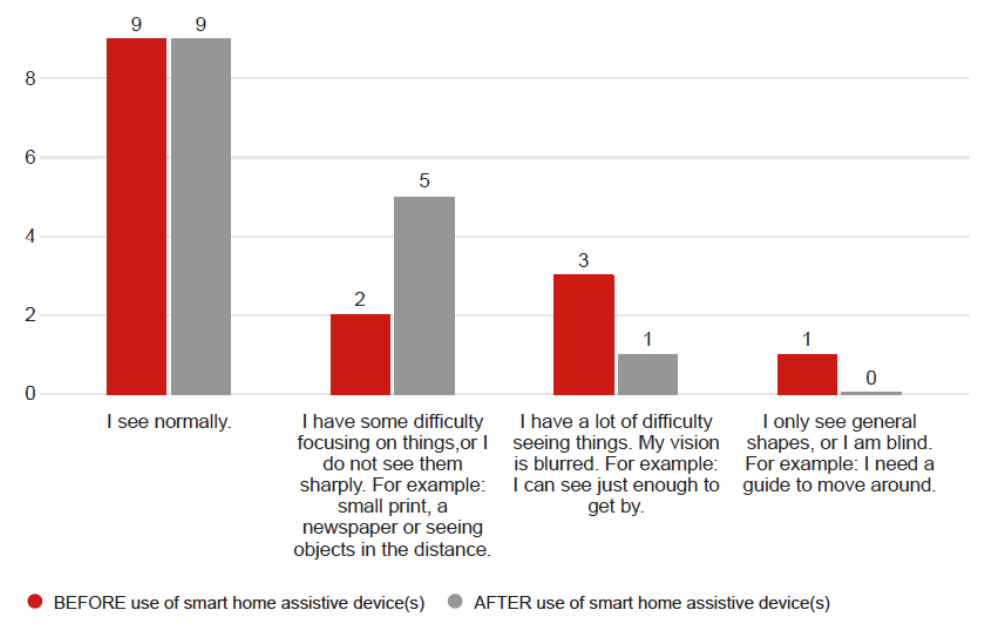

Source: Authors. 
Figure 13 indicates that satisfaction with hearing, whilst normal for many, was significantly improved for most respondents.

Figure 13: Perceived change with auditory activities

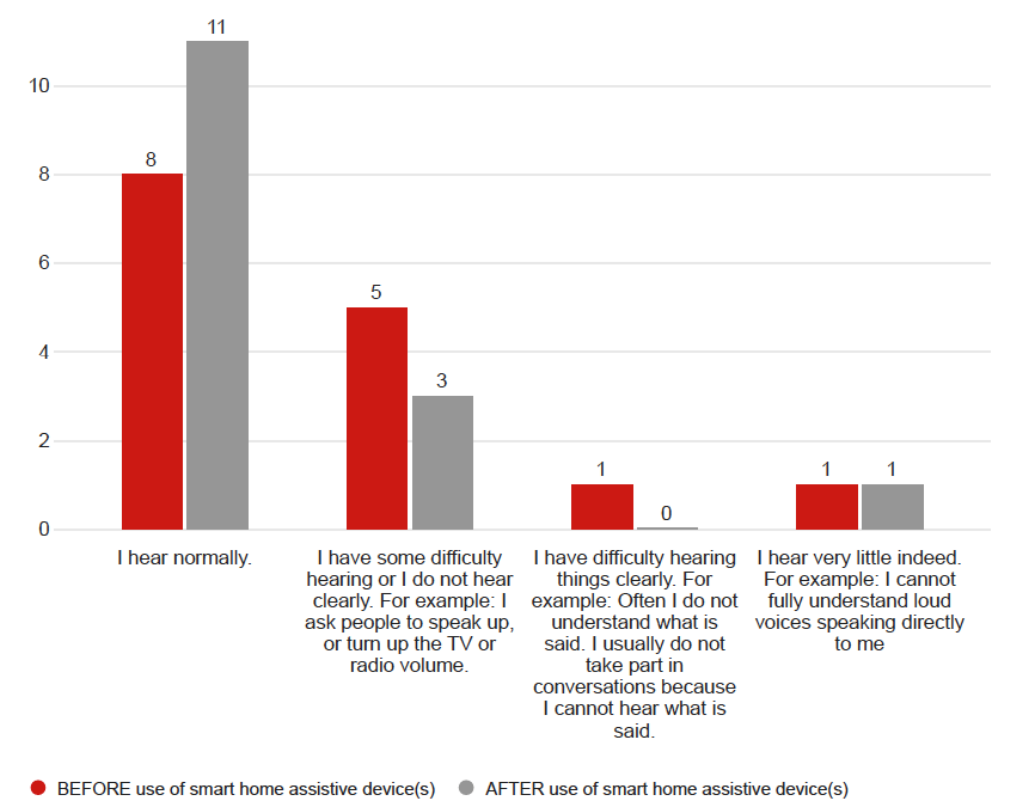

Source: Authors.

Figure 14 illustrates the perceived change in communication abilities. While all respondents stated that they could communicate adequately and that this remained unchanged, their speaking and understanding was improved. A few respondents experienced some frustration with being understood. This is unsurprising given that some smart voice control systems still struggle with some commands and rates of speech.

Figure 14: Perceived change with communication abilities

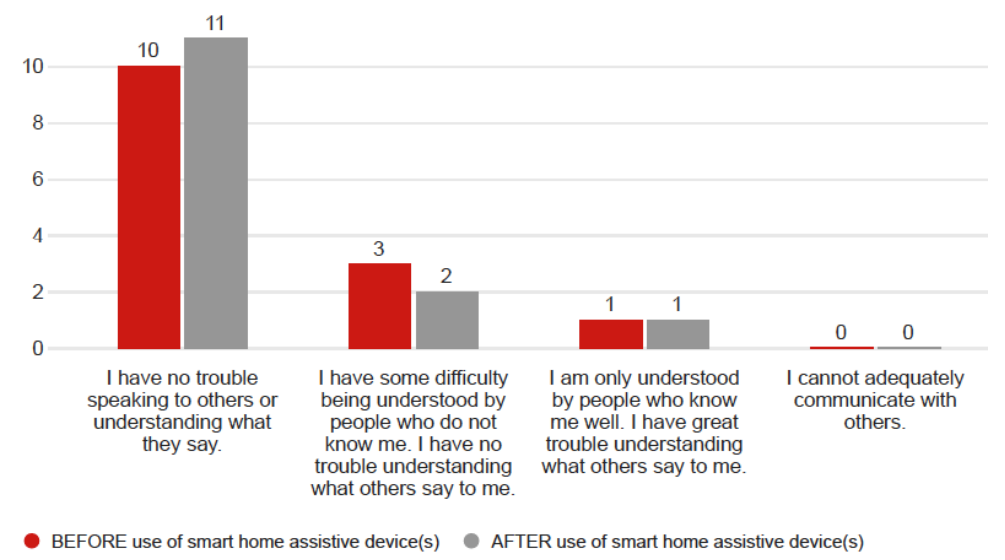

Source: Authors.

Figure 15 indicates that while a few respondents had serious sleep difficulties, the smart home AT had a detrimental impact on sleep quality, increasing interruptions for a number of respondents. 
Figure 15: Perceived change with sleep quality

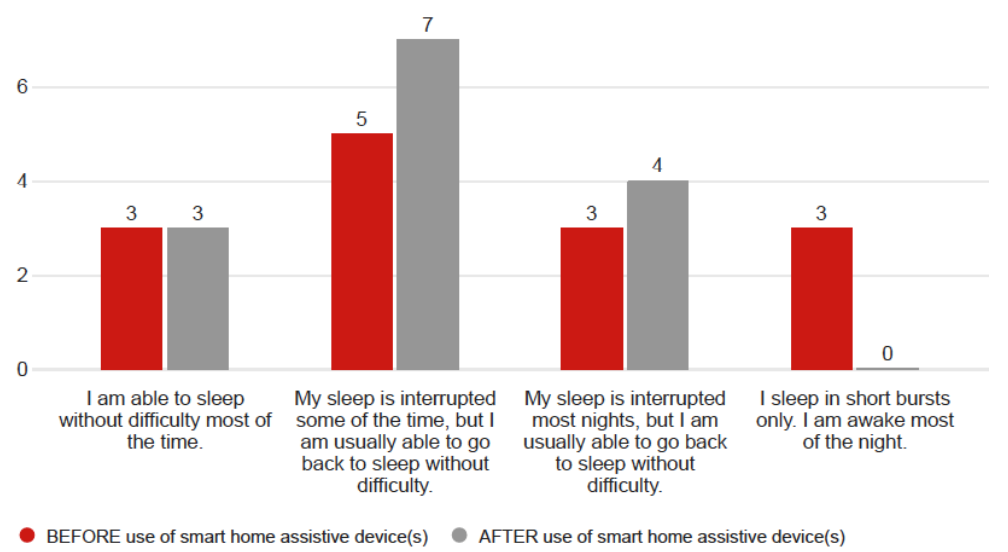

Source: Authors.

While most respondents had no pain before and no change after, Figure 16 illustrates that severe pain was reduced by installation of smart home AT for some, but moderate pain increased for others. Exactly why this so likely depends on the type of smart home AT purchased. For instance, increased use of music or Transcutaneous Electrical Nerve Stimulation (TENS) has been shown to reduce movement-evoked pain and fatigue (Dailey et al. 2019). However, for some conditions where chronic pain is present, pain may increase over time particularly if combined with feelings of social isolation (Karayannis et al. 2019).

Figure 16: Perceived change with pain experienced

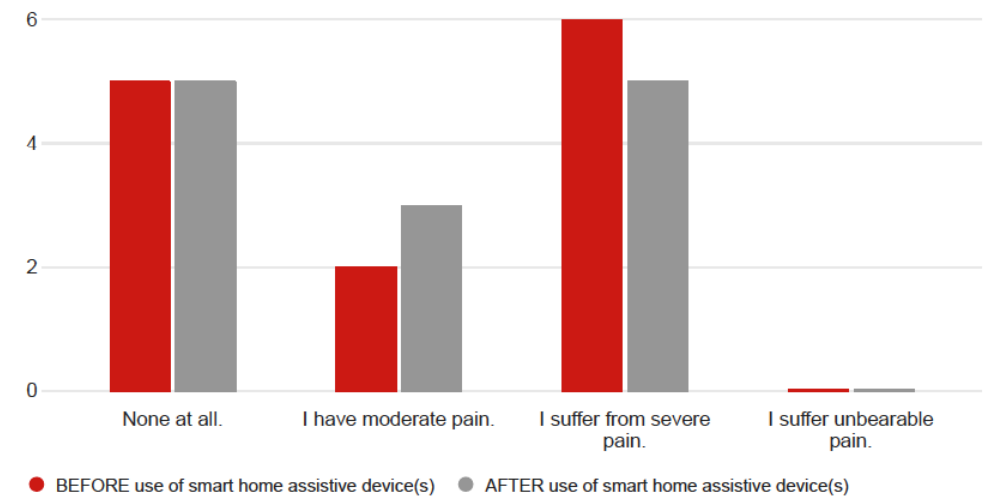

Source: Authors.

\subsection{Economics of smart home AT}

Economic evaluation has become one of the principal methodologies in outcomes research within the health care field. It is increasingly used to examine new and emerging technologies. The economic component of this project aimed to develop cost-effectiveness modelling based on the consumer smart home AT survey data collected in Phase Two, combined with supplementary figures from the literature review. The project approach was designed in the context of rapid market growth in smart home AT products and initial Australian research undertaken in 2012 that identified the need for greater policy support (Department of Communities Child Safety and Disability Services 2012). This initial research reported clear recommendations for state and territory governments and the Australian Government to develop policy interventions, facilitate health promotion campaigns focused on smart home AT benefits, identify products to benefit people living with a disability, and facilitate system wide introduction through new funding initiatives. As it has been several years since these recommendations were published, it was anticipated that the current project would be examining progress and assessing evidence of smart home AT uptake and consumer outcomes. 
Given the unexpectedly small AQoL survey sample, we explored whether there may be relevant proxy estimates of quality-of-life in the published literature for potential integration with the economic modelling. The literature review did not identify suitable smart home AT research for relevant study populations and for this reason the modelling is based exclusively on the small user survey sample. The user survey illustrates the variation in cost ranges, additional out of pocket expenses in the case of Australian Government supported homes, as well as ongoing maintenance for annual services and product upgrades. The total cost of ownership of smart home AT is based on expected useful life of products and the interoperability and upgrade pathways. For these reasons, the data sources for the economic modelling are similarly constrained and accordingly, the figures are provided only for preliminary reference. This finding has direct implications for the economic component of the project as the survey does not provide sufficient data to establish product profiles, support statistically significant analyses of outcomes, or investigate the important examination of consumer sub-groups.

Notwithstanding these data limitations, the economic perspective for smart home products reflects the emerging AT market, with high variation in product ranges, specifications, functionality, quality and cost. The economic assessment of AT may therefore develop quickly in the rapidly growing smart home market through integration with new cloud-based technologies. This accelerating market development is increasing potential target populations across aged care and disability settings.

Economic assessment of this emergent highly diverse market requires an articulation of: product profiles, eligibility of consumers for government support packages, and consideration of consumer need. Without this raw data as context for AT economic evaluation it is difficult to establish core product sets as baselines for comparison. This project has found that adoption of smart home AT products is very limited through government supported programs for disability or aged care. In this context, there are important initial questions about the potential direction of the smart home AT market. What are the target populations and potential scale of the impact if growth trajectories and effectiveness of AT are realised? What is the scope for policy intervention to stimulate adoption, incentivise the market and provide guidance across safety, privacy and rapidly growing smart home product ranges?

It is well recognised in health economics that maximising population level health outcomes frequently depends on the stratification of target risk and benefit-based sub-groups (Kohli-Lynch and Briggs 2019). In this context, while there are smart home AT parallels to the assessment of new technologies used in healthcare, a starting point is to identify defined product specifications for target groups of consumers. In the case of smart homes, the intervention is complex and crosses over multiple technologies that are dynamic and developing quickly. The physical devices are a focus, but the ubiquitous expansion of cloud-based platforms underpins low-cost integration of applications and devices to build virtual smart home environments.

This section presents the preliminary economic analysis of the user survey data, the indicative smart home AT costs and potential quality-of-life benefits. These were developed into modelling scenarios and preliminary cost-effectiveness examples as a novel and positive aspect of this research. In line with preceding sections of this report, the smart home component of AT is yet to be clearly defined and established in government support program eligibility. This project is being undertaken in the context of policy reforms in disability and aged care services, which are developing new flexible funding initiatives that may include increasing smart home AT. These emerging technologies, as for established physical types of home modifications, may similarly provide associated physical and mental health benefits resulting from sustained independent living at home.

\subsubsection{Smart home AT product costs}

The user survey questions stratified smart home AT products into five cost ranges from below $\$ 200$ to above $\$ 2,000$, as shown in Figure 17. Around a third of respondents indicated products below $\$ 200$ reflecting, for example, entry level off-the-shelf smart home assistant devices and perhaps basic smart lighting. Around a third of consumers ranged between $\$ 200$ and $\$ 2,000$ in line with the flexibility to customise product profiles and functionality. The remaining third of consumers reported the higher cost bracket above $\$ 2,000$, which is an open category to capture higher end installations. Consistent with focus group themes, the eligibility process for Australian Government supported smart home AT is subject to case-by-case interpretation of consumer need and resulting approved 
Australian Government support is currently negligible. The dependence on self-funding is reflected in most responses $(83 \%, n=25)$, with the remaining consumers receiving some level of Australian Government support funding $(17 \%, n=5)$.

Figure 17: Total upfront cost of assistive devices with and without government support

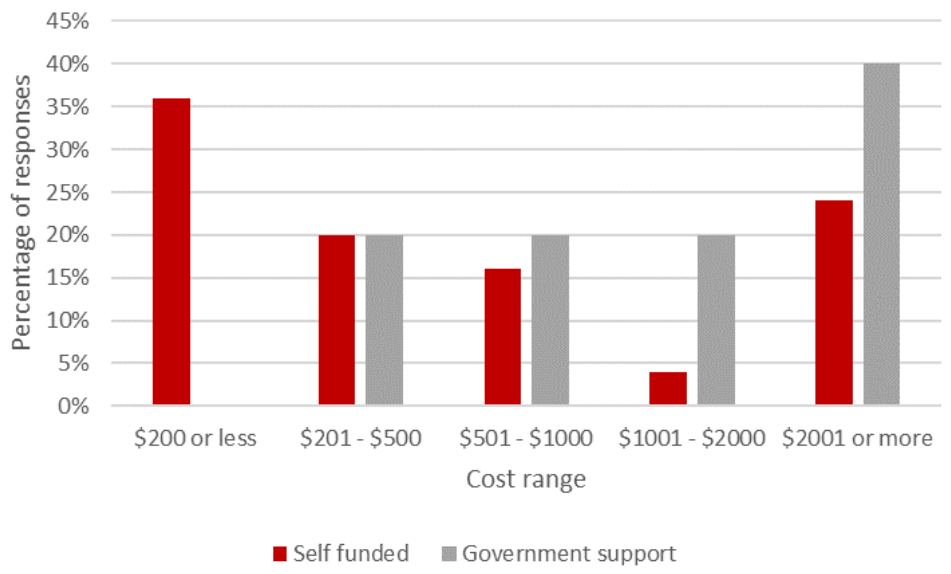

Source: AT user survey ( $n=30$, no support $n=25$, support $n=5)$.

The user survey identified the out-of-pocket cost of assistive devices. Although, with the majority of smart home AT being self-funded, this question only applies to a small proportion of consumers-indicatively below 20 per cent of consumers. As shown in Figure 18, of those receiving Australian Government support, there were reported cases of higher range out of pocket costs of $\$ 1,000$ and above $\$ 2,000$. This suggests that even for the small number of Australian Government supported smart homes, the installations were jointly funded projects. The user survey responses are again consistent with focus group discussions where smart home AT was primarily self-funded.

Figure 18: Out of pocket cost of assistive devices with and without government support

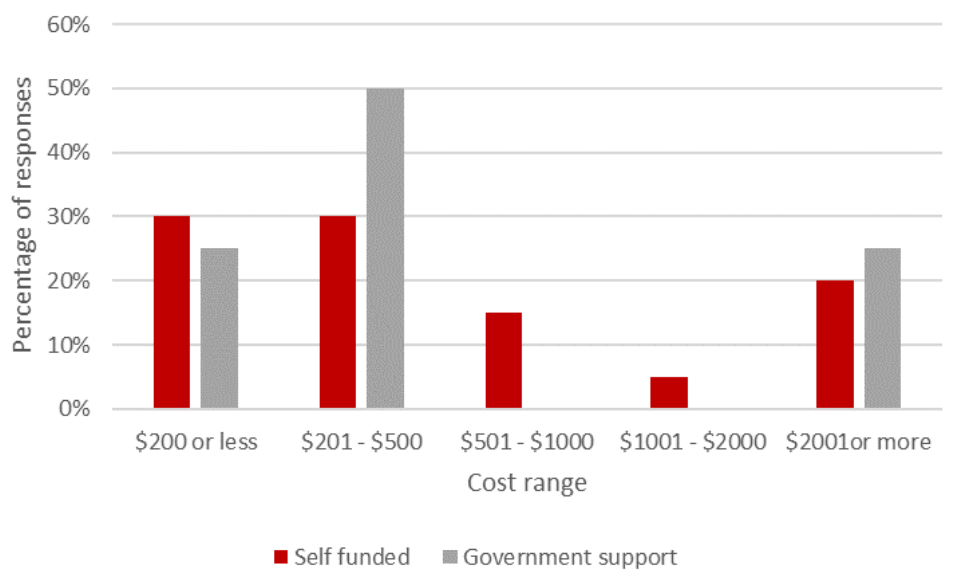

Source: AT user survey ( $n=24$, no support $n=20$, support $n=4)$.

Following smart home initial implementation, the user survey investigated ongoing annual running costs, maintenance and upgrades, as shown in Figure 19. The responses reflect generally lower cost brackets below $\$ 200$ per annum perhaps covering cloud-based subscription services and other routine maintenance below $\$ 500$ for both self-funded and Australian Government supported installations. 
Figure 19: Ongoing annual cost of assistive device with and without government support

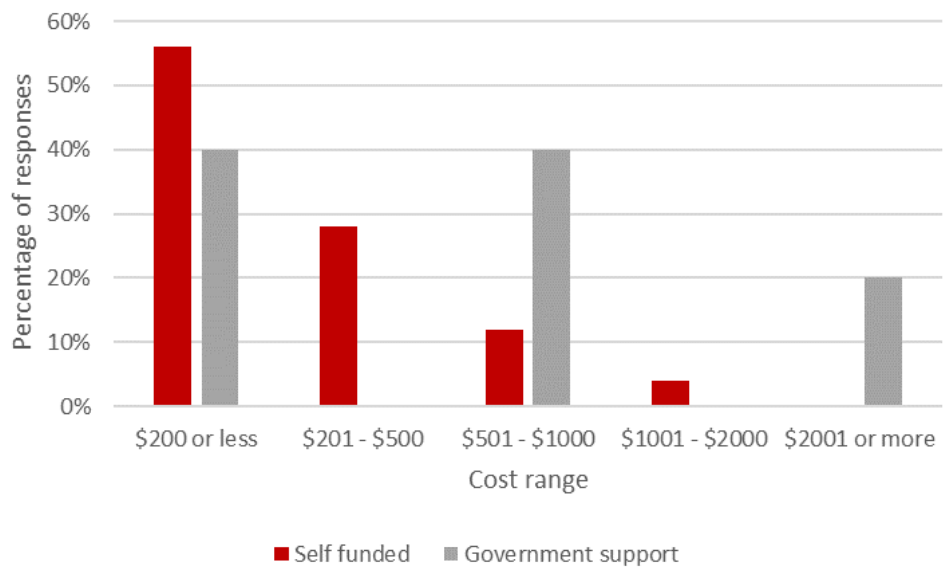

Source: AT user survey ( $n=30$, no support $n=25$, support $n=5)$.

The reported cases of annual cost above $\$ 2,000$ per annum may reflect significant upgrades or require clarification around how ongoing costs are defined (to separate system maintenance from the expansion of functionality or the addition of extra sensors). Given the rapid development in smart home AT and the inherent uncertainty of useful life, the economic perspective is potentially shorter than physical AT such as structural home modifications. For this reason, the economic modelling presented in following sections is based on a conservative five-year base case where benefits need to be realised within a timeframe aligned with expected product longevity.

\subsubsection{Housing provider and commercial smart home segment}

The research team consulted with commercial smart home suppliers to develop typical AT specifications for housing providers. The sector is developing specialised commercial divisions focused on assistive, independent, and aged care living capability. A typical requirement brief from NDIS providers includes a complete home automation system for an independent living and carer set-up in a high-rise development with potential open integration to multiple apartments. The outline of requirements generally integrates control of building intercom and access, doorways, blinds, lighting, heating and cooling with an overarching focus on ease of use, interoperability across technology platforms and accessibility for maintenance. A typical requirement specification is outlined in Table 13.

Table 13: Indicative smart home provider requirements specification

Heating and cooling operable via home automation system; temperature on/off function mandatory with preferred full temperature control through sensors

Intuitive user interface with the ability to be customised to suit the ability of tenants

Interface control via tablet with wall-mounted charging; operational whilst charging

Home automation and technology to operate on Apple iOS or Android with a single interface, i.e., does not require switching between applications

Safety integration connected to base building fire alarms with manual override and unlocking to ensure life safety requirements

Automated window blinds on command including full open/close and percentage open

Automated lighting with on/off function and dimming

Security with video intercom from apartment to building main front door with ability to view, communicate and release door lock through remote interface such as an iPad

Provision to add automatic door openers to individual apartment if needed

Provisions to program pre-determined 'scenes' such as 'return home' or 'exit home' including grouped settings for lighting, heating (on/off) and windows 
Facility for motion sensors to be installed later in nominated rooms (for example an accessible bathroom and bedroom) to detect and monitor motion or movement, and capability to communicate an alert signal

Ability to communicate with support person via integrated devices including two-way voice and alert function via fob, iPhone, iPad, etc. with capacity for escalation of alerts

Support person communication to allow for main station with an additional mobile component should the support staff be with a tenant in the building

Automated alert functions to escalate to different individuals if no response is detected

Home automation and communication technology must have minimum two-hour backup power to ensure safe use of the apartment

Assistive technology solution must be open platform to enable interface with a variety of different components

Ability for assistive technology to be controlled remotely to allow for system maintenance

Source: Harvey Norman Smart Home Automation Division. (2019) Unpublished raw data.

From consultation with a commercial smart home automation provider Harvey Norman in 2019 the typical AT product profile to meet these requirements includes:

- Amazon Echo Show and Show 5 with premium smart speaker 5.5" touch display

- Dimmer switches for control of all lighting with welcome and goodbye scenes (three units)

- Power Double general-purpose outlets with smart cards and controllers (three units)

- Motorised blind controllers (three units) and air conditioning controllers

- Netgear Nighthawk Wi-Fi Router for home automation and general use

- Installation, consumables and site project management.

The total cost of this assistive technology package is quoted at $\$ 6,190$.

Reflecting the variation in user requirements and product profiles, additional items may be included:

- Wall docked iPad Air, charging sleeve and installation - \$1,099

- Biometric door access controller, strike and installation - \$1,249

The cost of electrical wiring will vary depending on integration to power supply backup and optional alarms, video, door openers or nurse call systems. We have assumed an indicative cost of $\$ 2,200$. The complete installation including automated door access and iPad is $\$ 10,738$.

\subsection{Smart home benefits}

In line with the large and growing product sets, the value of smart home AT is implicitly driven by the ability to customise technology and design systems that meet the individual goals and needs of consumers across diverse housing circumstances. There is growing importance to develop innovative ways to employ AT with a range of potential interrelated benefits across mental health, relationships and independence. This section presents findings from the limited user survey illustrating examples of potential benefits.

\subsubsection{Improved quality-of-life}

The user survey included the Assessment of Quality-of-life (AQoL-4D) instrument, which is a short 12 multiple choice questionnaire covering four smart home AT relevant dimensions of independent living, mental health, relationships, and senses. This instrument was used as it is validated for health-related quality-of-life utility scores and estimation of Quality Adjusted Life Years (QALYs). Although based on a small sample ( $n=15)$, the results reflect quality-of-life benefits that may result from wellbeing improvement through increased independent living at home, increased mobility, reduced anxiety, reduced fear of falls and improved general physical and mental health. 
To begin with, the quality-of-life survey results indicated consistent improvements across all dimensions of the instrument, as shown in Figure 20. The scores are standardised with zero being the lowest and one being the highest measure. This reflects improvement in scores before (red) and after (grey) installation of the smart home AT products. Independent living was the lowest scoring dimension before AT installation and showed the largest relative increase. Although the results are based on a small sample, the responses are consistent with focus group feedback that smart home AT improved consumer wellbeing and feelings of independence.

Figure 20: AQoL-4D standardised mean scores before and after AT installation

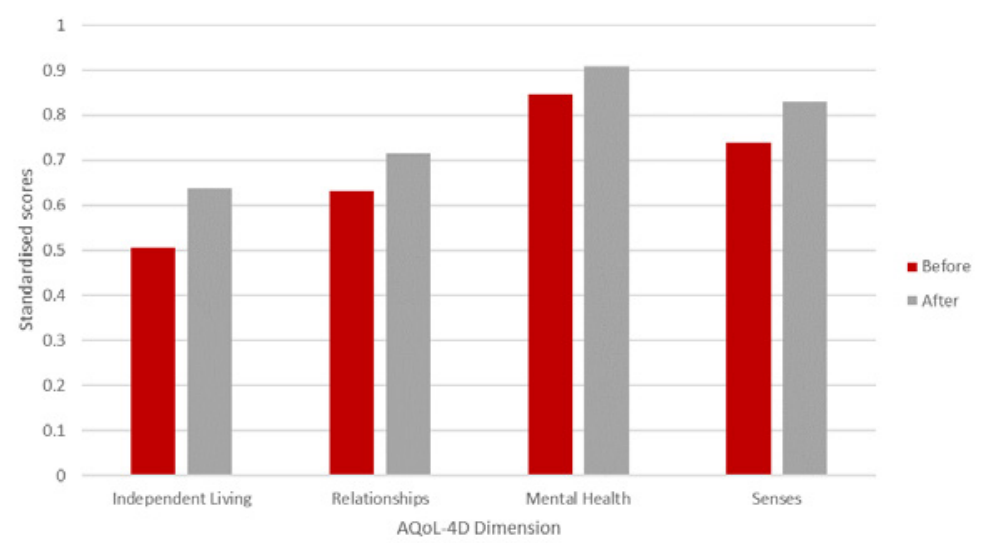

Source: AT user survey (AQoL-4D responses $n=15)$.

Other quality-of-life dimensions, including relationships, mental health and senses, had higher starting point scores prior to smart home AT adoption. All consistently increased following the introduction of respective products.

Further assessment was undertaken across two sub-groups based on the cost of installed smart home AT for above and below $\$ 500$, as shown in Figure 21. Consistent with the overall sample, the higher and lower cost category responses indicate improvement across all dimensions, suggesting quality-of-life benefits across a wide range of products. The independent living dimension suggests consumers with lower levels of independence tend to implement more comprehensive higher cost installations, and those with higher independence may be more commonly using entry level technologies.

Figure 21: AQoL-4D standardised mean scores before and after AT - by cost category

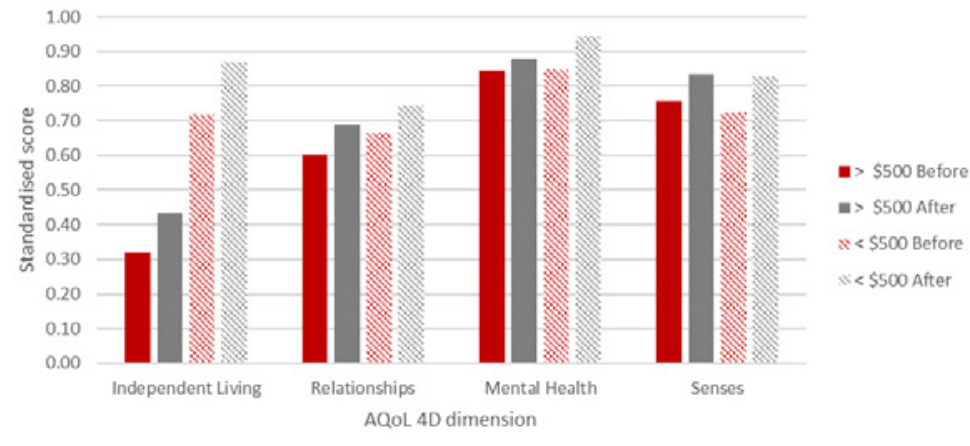

Source: AT user survey (AQoL-4D responses $<\$ 500 n=7,>\$ 500 n=8$ ).

Lower cost AT products are also supporting quality-of-life benefits across all dimensions, plausibly reflecting aspects such as an improved feelings of security, safety and connectedness. Those using more substantial AT generally reported lower quality-of-life scores before installation of their smart home products compared to the lower cost group, plausibly reflecting their higher relative need. 


\subsubsection{Improved quality-of-life and extended independent life}

In addition to potentially improved quality-of-life, it is also plausible that smart home AT could contribute to extended life expectancy. For example, in 2012, there were 462 deaths in NSW reported as being the result of falls, of which 43 were the result of slipping, tripping, and stumbling (Australian Bureau of Statistics, 2012). This category of falls may align with situations where the devices that are part of a smart home ecosystem such as smart lighting and fall detection sensors could help. The benefits perceivably include reduced risk of falling, less severe falls as well as reducing or eliminating the risk of remaining injured and undetected following a fall. The benefits of reduced fall risk are difficult to quantify but may contribute to improved trajectories stemming from avoided injury or faster treatment and recovery. In addition to sustained quality-of-life where falls are reduced, the implications are that falls at home are associated with higher rates of transition to residential aged care (Watson et al. 2011).

\subsubsection{Smart home efficiency}

A core characteristic of smart home technologies is the connectedness to cloud-based platforms, other devices and by extension, potentially to a group environment building. These aspects were discussed with smart home commercial providers during the project and illustrate potential efficiency benefits for individual home residents and collectively.

To begin with, smart home light and temperature sensors enable automated blinds and air conditioning to be adjusted throughout the day for shading and optimal settings. Data were not available on specific savings, but this functionality plausibly contributes to energy and related cost savings for individual properties. Smart home AT also may be configured to monitor individual apartment power for more accurate usage and billing within buildings that may use more approximate allocation measures for collective energy costs.

Additionally, on a collective scale of smart homes, the technologies can integrate residents with overarching smart building capabilities. This may include for example, collective solar panels on a building roof, distribution and measurement of output for residents and proportional allocation of efficiency gains and cost offsets. This is in addition to potential efficiency gains across individual homes through dynamic automated sun screening, ventilation, heating and cooling through the year. A typical profile for this type of integrated energy management system includes a communication module, energy sensors across five separate loads, set-up, application programming and installation and is quoted at around $\$ 1,100$.

A further potential smart home efficiency may emerge in supported care where cloud-based systems can be integrated into virtual settings. This may include a communal nursing capacity through on site or local monitoring for aged care or disability settings, for example across a group of several apartments. This introduces new alternative approaches to share and coordinate flexible levels of supported living, with the potential to pool costs across a group of residents.

\subsubsection{Economics of improved safety outcomes}

Health economics is concerned with safety in terms of costs related to the reduction of injury and potential health improvement, reduced anxiety, risk of falls, and so on. Nevertheless, the relationship between sustained independent living and the feeling of improved security is complex, and there is no standardisation in safety valuation. Ongoing research is examining the aspects of valuing safety and methodologies for the valuation of safety, theoretically and empirically (Perry-Duxbury et al. 2019). Smart home AT may contribute to general wellbeing and independent living through monitoring, lighting, and integrated communications.

\subsubsection{Economics of improved physical activity}

Insufficient physical activity is a well-recognised risk factor for healthy ageing. Approximately one-third of people over 65 years report being sufficiently active, one-third insufficiently active and the remaining one-third report being inactive (Australian Institute of Health and Welfare 2018). Smart home sensors may support better monitoring of activity, helping establish goals and sustained outcomes. 


\subsubsection{Economics of mental health outcomes}

In addition to physical health, remaining mentally active into older age is increasingly well recognised as important to social and mental wellbeing, maintaining cognitive function, and supporting independence. Smart home integration may contribute to and monitor reading, writing and doing puzzles as helpful activities to keep mentally active (Australian Institute of Health and Welfare 2018). Healthy ageing involves more than just promoting good physical health. Social and mental wellbeing are also important determinants for a high-quality life into older age.

\subsubsection{Economics of healthcare monitoring}

A future potential benefit of smart home AT lies in data-driven decision-making, providing opportunities for proactive, rather than reactive, care. Some segments of home healthcare monitoring have been established and trialled with mixed results in terms of effectiveness and affordability. Telehealth models, for example, establish various levels of video consultation with doctors and nurses combined with connected devices such as blood pressure monitors.

Smart home AT may support selective integration of targeted monitoring at cost points where benefits feasibly outweigh establishment and maintenance costs. Healthcare monitoring is an example of smart home AT which provides potential niche application for targeted consumers that may benefit from well-designed monitoring. While academic research in these select areas remains to be developed, anecdotal evidence is available through international news media outlining significant benefit in aged care settings for medication monitoring and management (The Economist 2020). For example, in Finland, the decision has been made to no longer develop residential aged care facilities and instead to support people in their own homes through telemedicine and community nursing. The approach in Finland is 'home is best' for everyone, at any age, even in cases of people living independently with dementia. The Helsinki municipal home care program is reported to support around 4,000 frail people with smart home AT, including GPS wristbands, fall detectors, alarm buttons and a phone to care workers.

The Finish experience illustrates the potential economic and healthcare benefit of robotic dispensing cabinets that automatically dispense medications on a predetermined schedule and issue reminders to users (Kangasniemi et al. 2019). Whilst smart home AT does not work in all cases, 80 per cent of end-users were able to reduce or stop medication related visits by nurses with cost savings resulting from the reduction of visits from 30 to four per month per person. More recently, the advent of the global COVID-19 pandemic has accelerated the necessity of communication and collaboration remotely and at a distance. Smart home AT is playing a major part in aged, disability and social care. Its rapid deployment has aided significantly in balancing the dual objectives of social distancing and preservation of economic activity, while facilitating the overarching suppression strategy (Smith et al. 2020).

\subsection{Economic modelling}

In line with the methodology, the aim of this project was to examine new preliminary data on smart home AT products, combined with initial outcomes from the user survey and in-depth case studies and focus groups. In this context, the economic model was developed to provide a framework to assess the available data and examine the: variation in costs and AT products; inherent heterogeneity in consumer needs; changing circumstances; eligibility for government smart home AT support; and capacity to self-fund the new technologies.

The modelling approach presented in this section to examine smart home AT reflects established techniques in health economics and formal processes for Health Technology Assessment (HTA) developed in recent decades in Australia and internationally. The assessment pathways for health technologies in healthcare are typically evaluated against an established comparator intervention, for a targeted population based on assessed need or diagnosis. Health economic methods assess promising, quickly developing technologies that are increasingly aimed at relatively small groups, such as expanding areas of personalised medicine driven by advances in genomics. 
For the emerging smart home AT market, the initial phases of economic assessment need to articulate specific product sets and target populations expected to benefit from the intervention. The findings from this project have highlighted the limited uptake of smart home AT in aged care and disability settings. The lower-than-expected user survey response, despite distribution through targeted national networks, may reflect variations in the understanding of what smart home AT is and how it could assist consumers. Despite the limited response, it is plausible that a larger sample may show similar characteristics in variation across product profiles, consumer circumstances and needs. The modelling undertaken outlines the available data, scenarios of products and outcomes and the perspective for ongoing development across policy, consumer advice, eligibility for government support and the development of an evidence-base for target segments.

Smart home AT upfront investment and ongoing costs reflect a rapidly growing and changing market. Economic timeframes need to align with relatively short-term horizons in some cases, where products may need upgrades or new components. The cost and timing of installation are then aligned with measured benefits, often lagged and diffused over many years, including outcomes that are difficult to measure financially, such as quality-of-life. As outlined in the previous section, smart home AT benefits are likely to include contributions to improved or sustained health, avoided or reduced ongoing risks, and resulting extended life at home.

\subsubsection{Health-related quality-of-life}

The preliminary quality-of-life results from the user survey presented in section 3.5 indicate a broad positive improvement in quality-of-life. These findings, while based on a limited sample, are consistent with feedback reported through the focus groups and case studies. This feedback suggests that smart home technologies support improved independence, activity, social and community connectedness and, as a result, potentially improved physical and mental health outcomes.

Although there is an ongoing debate regarding the suitability of QALYs, including for evaluation of AT, they are widely accepted as the best available measure to standardise health adjusted life year modelling (Persson and Husberg 2012). Further, despite well-known limitations with QALYs, they are central to health technology assessment frameworks in Australia and internationally. There is limited published literature related to the economic evaluation of AT, and much is dated and focused on physical and mechanical AT rather than new smart technologies for the home. Early research applied simulated cost-utility analysis (CUA) to assess the cost-effectiveness of electric versus manual wheelchairs, demonstrating the estimated quality-of-life improvement from electric transition and presenting the usefulness of CUA in this setting (Brodin and Persson 1995). A separate study used CUA to assess powered mobility devices for older people and found the devices likely to be cost-effective, although the quality-of-life scores were not significant (Hagberg et al. 2017). The overarching characteristic of quality-of-life instruments is the assessed dimensions, commonly mobility, independence and mental health which are equally valid for assessment of new smart home technologies.

\subsection{Effectiveness and cost-effectiveness}

The cost-effectiveness estimates presented in this section have been developed as a preliminary framework to assess the scale of smart home adoption, and to provide estimates of plausible cost savings and quality-of-life benefits that could potentially be provided. The initial economic work examines available data in the context of substantial variation, rapidly changing products and inherent heterogeneity in consumer circumstances and need. In this overarching context the preliminary framework reflects current available data as a basis to position potential pathways for policy, further development of funding sources and ongoing work needed to build the evidence-base for smart home AT.

The effectiveness and related cost-effectiveness of AT is associated with a range of potential benefits that are plausibly and anecdotally linked with independent living, such as mobility, social activity, and mental and physical health. These aspects are not articulated in the modelling as there are no primary data available to indicate benefits resulting from specific smart home product profiles for targeted cohorts of eligible consumers. These aspects are plausibly reflected indirectly through indicative improvements in quality-of-life outcomes. 
The core elements of the economic model include potentially reduced risk and cost of falls, avoided or delayed transition to residential aged care and avoided decline in quality-of-life associated with smart home assisted support. Due to the skew towards high volume and relatively low-cost of smart home technologies, and the trend of improving technologies at lower prices, smart home devices are potentially providing cost-effective outcomes across these dimensions for specific sub-groups of consumers.

\subsubsection{Smart home product effectiveness}

As presented in previous sections, the user survey AQoL questionnaire indicated the increased quality-of-life across all dimensions, including independent living, relationships, mental health and senses. The survey AQoL results were standardised to provide utility scores where zero represents the lowest and one represents the best possible quality-of-life, as illustrated in Table 14. The total survey group $(n=15)$ suggests an indicative quality-of-life increase of 0.13 following introduction of the smart home AT.

Table 14: AQoL 4D overall utility scores before and after AT - by cost category

\begin{tabular}{|c|c|c|c|c|c|c|}
\hline Study group & $n$ & Mean & S.D. & Min & Max & Qol change \\
\hline Total before & 15 & 0.31 & 0.33 & -0.01 & 0.98 & \\
\hline after & 15 & 0.43 & 0.35 & -0.00 & 0.98 & 0.13 \\
\hline$\$<500$ before & 7 & 0.41 & 0.38 & 0.01 & 0.98 & \\
\hline after & 7 & 0.58 & 0.36 & 0.06 & 0.98 & 0.18 \\
\hline$\$>500$ before & 8 & 0.22 & 0.28 & -0.01 & 0.64 & \\
\hline after & 8 & 0.30 & 0.31 & 0.03 & 0.79 & 0.08 \\
\hline
\end{tabular}

Source: AT user survey (Total $n=15,<\$ 500 n=7,>\$ 500 n=8)$.

The AQoL utility scores were also calculated for sub-groups of product cost below and above $\$ 500$ as outlined in the previous section (as shown in Figure 21). Although these figures are based on small sample sizes, they plausibly reflect quality-of-life benefits across product cost ranges. The lower cost below $\$ 500$ group indicated a mean AQoL score of 0.4 before introduction of the AT, with a 0.18 increase following implementation. The higher cost product group reported a lower AQoL mean score of 0.2 before their AT was implemented, reflecting a lower baseline and potential higher need for more significant support, with a 0.1 increase in reported quality-of-life following take up of the AT. While these are marginal increases, they are produced at modest cost points and therefore potentially represent cost-effective interventions.

The survey results illustrate the high variation in smart home AT costs and indicative quality-of-life benefits. The product cost profiles have been stratified in line with survey categories and an example NDIS scenario as a higher cost case. The economic Markov model was developed for each of the three cost scenarios with a comparative no AT representative baseline, as shown in Figure 22. The model has been specified with initial purchase cost and estimated maintenance over five years combined with mean AQoL results and standard deviations from the user survey, as shown in Table 14. This initial view provides a perspective of the profiles generated using model bootstrapping of the indicative product mean cost and variation combined with survey mean quality-of-life benefit and corresponding variation for each group. 
Figure 22: Indicative assistive technology costs and effectiveness by cost category

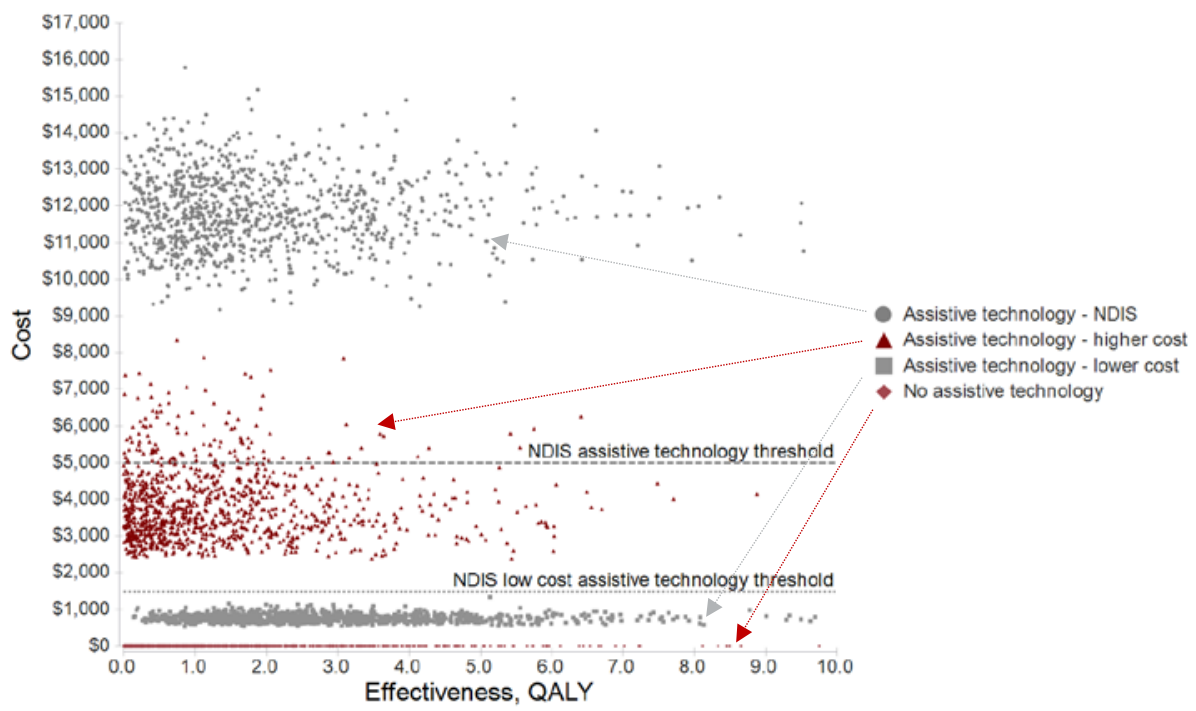

Note: Presented for illustrative reference, not precision results.

Source: AT user survey (Total $n=15,<\$ 500 n=7,>\$ 500 n=8)$.

The no AT baseline is shown as the lowest flat red sample of estimates at zero cost with variation across the quality-of-life axis reflecting the 'before' introduction of smart home AT AQoL scores with an indicative mean of $1.46^{2}$ and standard deviation of 1.56 QALYs. Moving up the figure, the next group is the low-cost category defined through the survey of below $\$ 500$. The low-cost example reflects variation in cost assuming $\$ 300$ initial product cost with a standard deviation of $\$ 100$ as well as $\$ 100$ per year for ongoing maintenance. The low-cost scenario incorporates indicative AQoL scores from the below $\$ 500$ user survey sub-group, plausibly reflecting a higher baseline for those choosing basic entry level products. Interestingly, responses suggest a potentially higher benefit from the initially higher starting point, perhaps indicating that relatively minor AT items may contribute to feeling more connected, safer and more independent. Equivalent low-cost physical AT items such as handrails have been shown to produce quality-of-life improvements through reduced risk or fear of falls (Bridge et al. 2016). This low-cost profile is below the NDIS AT threshold of $\$ 1,500$, providing a potential government funding source for selected consumers where eligibility is established.

The higher cost product profile is defined as a mean purchase and installation cost of $\$ 1,500$ with a standard deviation of $\$ 1,000$. This is consistent with the high variation in consumer need product range combined with an assumed $\$ 500$ per year for maintenance or product and software upgrades. As for the low-cost example, the AQoL benefit scores are incorporated from the higher above $\$ 500$ survey subgroup. The Markov model bootstrapped estimates reflect a mean cost over five years of $\$ 3,825$ with a standard deviation of $\$ 986$. The higher cost group reflect the slightly lower AQoL gain based on survey responses with a mean of 1.4 QALYs, represented by the slightly lower left clustering of the plot for this group. Above $\$ 1,500$ the NDIS currently requires individual assessment of eligibility which the focus groups indicated is perceived as difficult to confirm. Approved case numbers are negligible.

The final example cost profile of a typical cost per home in an apartment complex is based on products quoted through commercial smart home suppliers. This example assumes a purchase cost of $\$ 9,488$ with a standard deviation of $\$ 1,000$ and the maintenance or upgrades cost of $\$ 500$ per annum. The AQoL benefit is based on the total survey sample to include the largest group and a conservative estimate of potential quality-of-life improvement. As for each product scenario, the results are based on a small sample from the user survey $(n=15)$, with corresponding high variation in responses.

2 Variation around base AQoL 'before' score of 0.31 over 5 years, including assumed discounting of 3.5 per cent per annum. 


\subsubsection{Cost-effectiveness}

The economic cost-effectiveness model was developed using the cost and outcome scenarios presented in the previous section. The Markov framework specifies the mean costs and outcomes, as well as the variation using standard deviations for all parameters. One of the strengths of this approach is the support of rigorous methods to evaluate the joint uncertainty in all estimated model parameters through sensitivity analyses and established health economic bootstrapping resampling of probabilistic sensitivity analysis. In the case of smart home AT, the user survey data has been used as a preliminary analysis. The limited sample sizes and substantial variation are reflected in the dispersion of model generated point estimates and related confidence intervals. As described in the methodology, the user survey sample size limits the statistical significance of the cost-effectiveness estimates. In this context, the scenarios are presented as indicative preliminary results in combination with the case study and focus group components of the research.

The results of cost-effectiveness analyses are commonly presented on an incremental cost-effectiveness plane as below in Figures 23 and 24. The figures show the incremental increase in outcomes (in this case QALYs) along the $X$ axis, with positive increases of introducing a smart home AT intervention as positive scores to the right of the vertical reference line at zero. Similarly, the cost of the smart home AT is presented on the $Y$ axis as positive figures; this shows the introduction of devices versus no device. These figures provide a scatterplot of the bootstrapped (resampling with replacement) joint probability distribution combining the variation in costs and outcomes.

The cost-effectiveness scatterplots also indicate an assumed willingness to pay threshold value, shown as the sloped line to the right of the vertical zero axis. Based on the indicative costs from the user survey, these are defined as a low-cost case of $\$ 500$ (Figure 23) and a high-cost profile of $\$ 10,000$ (Figure 24). The threshold values provide a reference for price point per unit of outcome. For example, Figure 23 assumes $\$ 500$ per QALY, and it can be seen that the threshold line shows one QALY at $\$ 500$ and higher at $\$ 1,000$ for two QALYs. The interpretation is that cost-effectiveness point estimates to the right (lower) than the threshold line would be acceptable to funders or individuals self-funding smart home AT, as those points are indicating higher outcomes for an associated cost. An inherent strength of these methods is the ability to estimate the probability that an intervention is cost-effective, given characteristic uncertainty in costs and outcomes. In this case, the small survey data sample size is reflected in high variation in estimates and a large widely dispersed 95 per cent confidence interval shown as the ellipse. This shows clearly that these results are not conclusive, despite marginal positive outcomes.

Each AT cost category was run as an incremental comparison to the 'no AT' baseline defined before the smart home products were introduced. The first example profile is based on the low-cost scenario with products below $\$ 500$, as shown in Figure 23. This profile assumes a purchase cost of $\$ 300$ with a standard deviation of $\$ 100$, as well as $\$ 100$ per year to cover maintenance or upgrades.

Figure 23: Estimated incremental cost-effectiveness - low-cost scenario

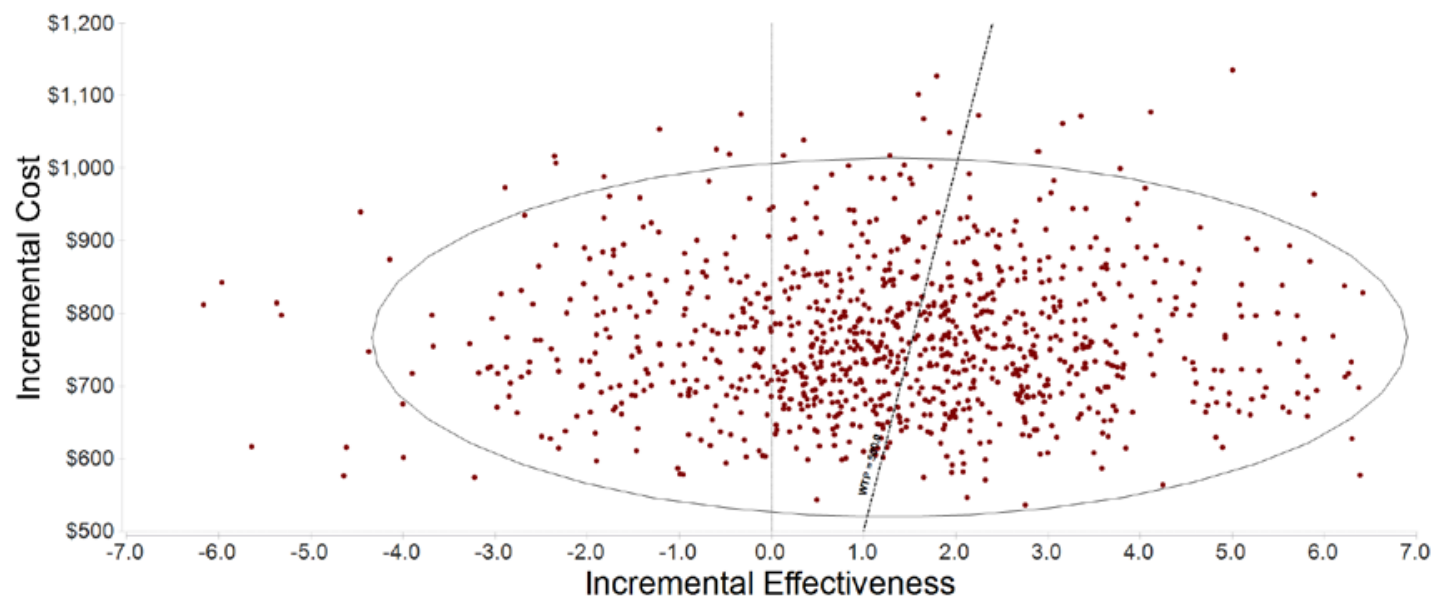

Notes: Ellipse indicates 95 per cent confidence interval; centre diagonal line indicates $\$ 500$ willingness to pay, vertical line at zero AQo L effectiveness for reference.

Source: AT user survey (Total cost $n=30, A Q \circ L=15)$. 
Combined, this establishes smart home AT cost over five years at around $\$ 700$ to $\$ 800$ with characteristic variation ranging from above $\$ 1,000$ to below $\$ 600$ as indicated by the 95 per cent confidence interval ellipse. On the horizontal outcome axis, the AQoL utility scores are shown as estimated QALYs with an incremental increase of 0.18 per year and related variation based on before and after user survey scores. As for the cost, point estimates for the QALY results are reflected by the 95 per cent confidence interval showing a wider range in outcomes as the elliptical boundaries.

The interpretation of the bootstrap plot of estimates is generally divided by a willingness to pay threshold, in this example it is shown as the diagonal line for an assumed $\$ 500$. This implies that the portion of cost-effectiveness estimates to the right of this line is acceptable and increasingly cost-effective the further the points are to the right. Higher willingness to pay thresholds per QALY gained would be shown as slightly more vertical dotted lines, resulting in a greater proportion of the plot being within an acceptable cost-effective range. For example, a willingness to pay $\$ 1,000$ effectively increases the confidence that this low-cost smart home AT scenario represents a costeffective intervention. The implicit issue is that this example is a general scenario including high variation in product profiles, costs and heterogeneity in consumer need. For this reason, specific sub-groups, where defined products are likely to be effective, may be masked within the results. This emphasises the need to articulate AT product profiles targeted for defined eligibility consumers which may then emerge with further data as smaller cost-effective ellipses located to the right of the plot, in scenarios where the broader less effective cases are reduced and smart home AT is potential of net benefit.

As highlighted in previous sections, these analyses are based on small survey samples and are presented for reference. It is plausible that additional survey data may have reduced variation and concentrated the confidence interval in line with these non-statistically significant increases in effectiveness. The bootstrapping, in this case, is reflecting the small sample size and emphasising that the results are not statistically significant. Individual point estimates are observed to be almost as likely to experience low benefit or decreased benefit (left of the centre line indicating zero effectiveness) as they are to result in a quality-of-life improvement.

The elliptical confidence interval may be indicating preliminary positive effectiveness but may also be the result of the small sample size variation or biases. For these reasons, the analysis is unable to determine a significant finding.

The model estimates across each cost scenario reflect the inherent variation in cost and the user survey-based quality-of-life outcomes. As the basis of an indicative NDIS scenario, the model assumes a mean cost of \$9,489 representing an indicative commercial example product profile, Figure 24.

Figure 24: Estimated incremental cost-effectiveness - NDIS scenario

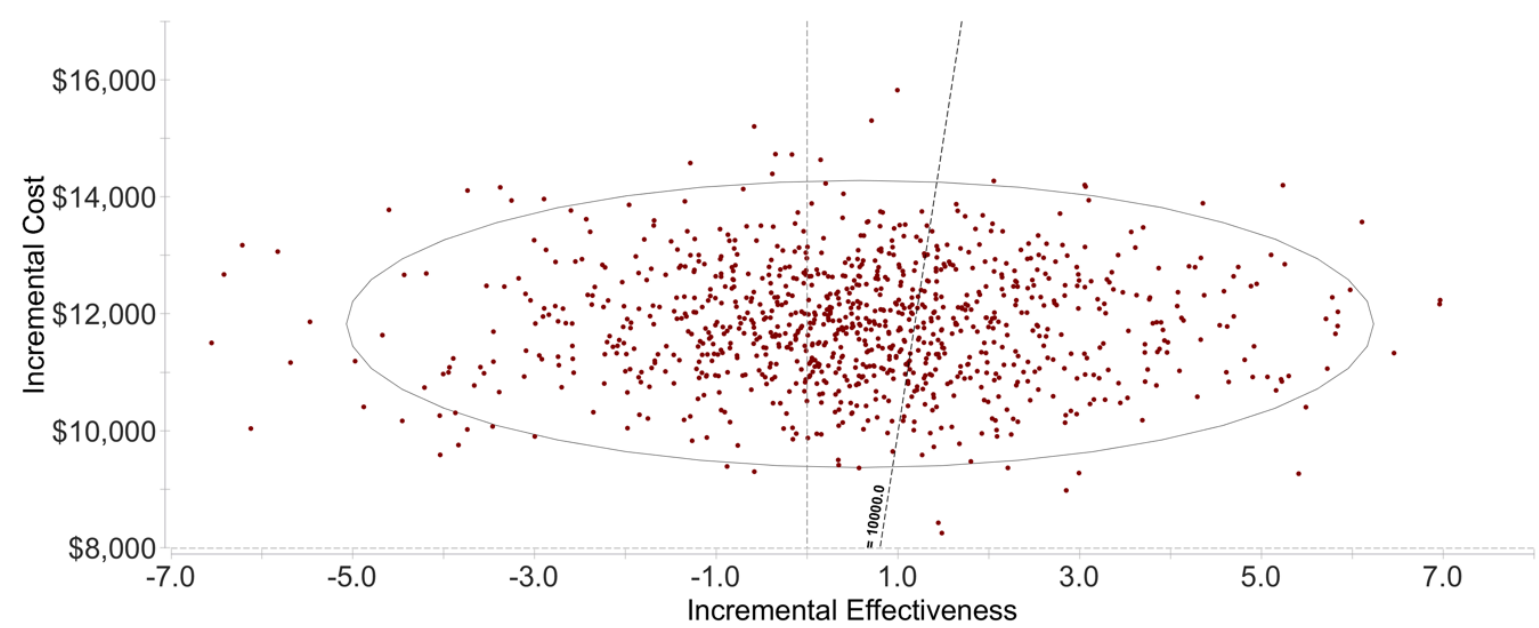

Notes: Ellipse indicates 95 per cent confidence interval; centre diagonal line indicates $\$ 10,000$ willingness to pay, vertical line at zero AQoL effectiveness for reference.

Source: AT user survey (Total cost $n=30, A Q \circ \mathrm{L}=15$ ). 
The model specifies a standard deviation of $\$ 1,000$ to reflect a proportion of consumers that may need specific access products such as automated entry, as in Figure 23 represented by the 95 per cent confidence interval ellipse. The quality-of-life benefit following the introduction of the smart home AT is based on the total survey sample of 0.13 with a standard deviation based on before and after implementation survey results. In this higher cost NDIS scenario, the willingness to pay threshold is assumed at \$10,000 per QALY, representing a level that is within health technology interventions funded through government subsidies. As for the previous low-cost example, this scenario reflects the characteristic variation in products, cost profiles and quality-of-life outcomes, and the need to articulate effective smart home AT products and consumer sub-groups to establish cost-effectiveness evidence for targeted scenarios.

\subsection{Policy development implications}

The sample size achieved reflects the fact that most respondents indicated a high degree of disability severity. It also echoes earlier, more general AT research which found that AT both increased with age and with the severity of disability related restrictions (Bricknell 2003) and implies that the user survey did, in fact, reach its intended audience. In policy terms, the limited sample size compared to the potential population of respondents could be indicative of several issues that future work needs to address. These issues include:

1. The response rate could be due to lower market penetration within this already disadvantaged sub-population group who are more socially and economically disadvantaged.

2. Previous research had noted that recruitment of people with cognitive impairments is low and slow without the opportunity to visit or phone participants (Lennox et al. 2005), as are those with significant mental health issues and depression (Brostow et al. 2015).

3. Online survey recruitment success declines with increasing age of subjects (Corey et al. 2018) and because many people with disabilities and older persons are incapable of consenting to the participation in scientific research (Faes et al. 2007).

4. Other researchers have suggested that unfamiliarity and distrust of research, plus the inability to differentiate between academic research surveys and commercial research, may have reduced participation (Allen and Roberts 2010; Becker et al. 2004).

5. Some non-response may have been due to lack of secure internet connection despite having smart home AT set-ups and given the level of impairments noted a much greater dependence on carers than expected (Patel et al. 2003).

6. Having a target group of respondents who require a high degree of assistance and may depend on a carer or guardian to fill out the online survey on their behalf means that future studies need to factor in additional assistance and incentivisation.

7. Recruiting via third parties is problematic, where additional challenges include lack of prioritisation, sociocultural issues, and being unable to offer either reimbursement to participants or draft findings to the thirdparty facilitators as a condition of the contract. Better targeting, motivating and reimbursing third-party survey distributors and participants may have yielded more respondents. For example, one established means to do this is to either pay for the time required or to provide early access to top-level data analysis that provides direct feedback about engagement and performance relevant to others.

Given recruitment was less than anticipated for the online survey. More incentives and support will improve similar endeavours. For instance, Becker et al. (2004) suggests that individuals with disabilities are more likely to volunteer if people they know, and trust explain the study to them and help them 'navigate' the online survey completion. They go on to recommend sharing openly the information obtained and inviting their perspectives on the meaning of findings as a part of the research process as this demonstrates a spirit of partnership. Additionally, as recommended by Brostow et al. (2015) a co-design process to maximise clarity and ease of use in conjunction with communityand home-based recruitment would be more expensive but would likely generate a greater participant response rate. Most of the respondents indicated that they self-funded their smart home AT purchases and received almost no government support. From this starting point the development of some targeted investment by all levels of the Australian Government in smart home AT may further promote appropriate uptake. 
Given that respondents had differing smart home AT profiles it appears that effective service set-up also needs to consider residential accommodation in tandem with individual needs. Given that a higher percentage of younger adults responded to the survey and there were slightly more men than women overall and less survey respondents from remote areas (less reliable internet, etc.), it may be that any additional policy to ensure greater equity may need to take these disparities into account. Given the smart home AT market currently receives negligible funding through government support programs but demonstrates potential to reduce in-home care and improve life satisfaction for people with moderate to severe functional impairments, this is something that future policy may want to explore.

The economic analysis is consistent with the positive quality-of-life results from the user survey. While based on a limited sample, the analysis is also consistent with other findings indicating that there are potential economic benefits across all dimensions, including independent living, mental health, relationships, and senses. There are potential economic gains not just for the Australian Government but for the industry in manufacturing and inservice improvement.

Nevertheless, the economic perspective for smart home products reflects the emergent nature of this market. Fast-changing markets require dynamic thinking and forward-looking policy solutions. This has become even more urgent with the COVID-19 pandemic. Housing and health economic policy directives need to urgently invest in further research and methodologies that systematically capture not just economic costs, shifts and benefits, but also the unexpected imperfections and failures. Importantly, future policy needs to coordinate and monitor outcomes in line with newer policy directives. Ongoing evaluation and monitoring are essential to better inform our emerging market crises responses, not just the factors that are relevant to establishing and maintaining smart home AT.

Given that people with disabilities and older people are some of the most vulnerable in society, the Australian Government needs to establish a monitoring and financing framework capable of robustly and sustainably dealing with the ubiquitous expansion of cloud-based platforms that underpin low-cost integration of applications and devices. Policy directives need to value flexibility and customisability of technology. Economic levers addressed by policy need to be capable of meeting individual goals, both low-cost and higher cost set-ups. Collecting data needs to encompass not only direct costs but also the less tangible costs gained from closely monitoring a range of potential interrelated cost savings across mental health, relationships and care. 


\section{Service provider and individual user views on Assistive Technology}

- Case studies provide a personalised and more nuanced understanding of why individuals and organisations that serve those who are ageing and/or have significant functional impairments differ in terms of their motivation, knowledge, skill, social support and financial capacities for assistive technology.

- Smart home AT is most effective where it is considered as a holistic system, including the relationship between technology, housing design and care.

- Education, training and support is essential for sustained uptake and effective use of smart home AT. This is a key funding gap.

- Inequities in access to AT encompass geographic and socio-economic dimensions, as well as inconsistency and uncertainty in funding across different funding schemes and jurisdictions.

- Commercial aged care and disability housing providers reported uptake of smart home AT policies where a market advantage was perceived. Social and community housing agencies found smart home AT out of scope and unaffordable.

- Management of risks (both perceived and real) is a central concern.

This chapter focuses on the question of what can we learn by asking end-users about their smart home AT purchases and outcomes? It elaborates on the survey analysis provided in Chapter Three by focusing on housing providers and non-government organisations from the five focus groups, combined with the four case studies, to provide a more nuanced and individualised experience relating to Research Question Three and Research Question Four. The focus groups and case studies presented here highlight positive feedback for the transformative impacts AT could bring for clients, including many examples of improved quality-of-life. 
The benefits of smart home AT emphasise the ability of the technologies to boost client confidence and a sense of independence across healthcare, aged care and disability settings. These themes reflect increased capacity for autonomy and improved wellbeing of consumers, increased peace of mind for families and carers, as well as potentially reduced need for care provision. The focus group feedback shows enthusiasm for what is sometimes seen as revolutionary benefits where smart home technology enables improved freedom personally, perhaps to new expanded areas of a property or gardens, or social connectivity aspects of consumer's lives. This chapter gives voice to the service providers and professionals in the area as well as end-users of the smart home AT. The analysis is organised under three themes related to end-users, housing and service providers, and managing the ethical implications that arise with smart home AT.

\subsection{End-user's experience of smart home AT}

Adoption of new technologies is typically a slow process because, when it is first introduced, a new technology is usually expensive, unfamiliar, and imperfect (Barreca 2000). Despite tech giants continuing to drive developments in the smart home technology sector with new and improved products, and the provision of these products in the mainstream market at lower price points, the adoption of smart home technologies as AT in Australia has, until recently, remained low. In 2013, a pilot study in 'Assistive Health Technologies for Independent Living' conducted by the Australian Academy of Technology and Engineering (ATSE) revealed a low adoption of AT and lack-lustre demand for mainstream AT (Tegart et al. 2014). With the introduction of Google Home in late 2017 and Amazon Echo and Apple HomePod in 2018, the adoption of smart speaker technology has increased considerably. According to Kinsella (2019), smart speaker technology is being adopted in Australia at a faster rate than in the US. Through their surveys they suggest in late 2018, 5.7 million Australians with online access owned smart speakers or 29 per cent of the adult population. Whilst they note that at first glance this number may seem inflated, they argue that this reflects the growing interest in Australia in new technologies. Globally, Australia leads smartphone usage (Marsden and Lee 2016) especially amongst people under 65 years. Nevertheless, because our ageing societies face mobility and isolation challenges, they have triggered an increasing number of funding agencies to promote a research agenda that calls for older people to take advantage of the capabilities of smartphones. However, previous research indicates that non-adoption and improper use of smartphones by older adults is common (Berenguer et al. 2016).

The technology acceptance model (TAM) introduced by Davis (1989) identifies two key factors in determining a users' acceptance and uptake of technology - perceived usefulness and ease of use. The TAM information technology framework provides a useful theoretical structure to compare users' adoption and use of emerging technologies particularly in the home. The TAM framework posits that perceptions of usefulness and ease of use are mediated by such things as individual differences, system characteristics, social influences, and facilitating conditions. Further, TAM theory posits that a person's intent to use (acceptance of technology) and usage behaviour (actual use) of a technology is predicated by the person's perceptions of the specific technology's usefulness (benefit from using the technology) and ease of use. As stated by Portz et al. (2019), end-users are more likely to adopt technology with that is usable, useful, desirable, and credible.

\subsubsection{Perceived usefulness}

Echoing the literature, and considering the adoption of smart technology by Australians, participants emphasised the benefits of AT in supporting independent living and the implications for wellbeing through increased autonomy, self-confidence and social connectivity. Advanced technologies, such as robotics, were seen to extend opportunities for independent living to a wider range of individuals living with disabilities and functional limitations. The availability of mainstream technologies were also seen to offer substantial benefits, described as 'revolutionary' and 'exceeding expectations'. Participants articulated how these improvements were directly attributable to the technology, that 'might not otherwise have been available'.

Smart home AT can be seen to enhance people's experience and ability to care by increasing autonomy and maintaining privacy, highlighting the importance of considering the subjective experience of care in relation to individual needs and preferences. 
Opportunities were seen to exist where smart home AT and housing design can be considered holistically. One participant described the impacts on autonomy and wellbeing for residents of a recently constructed smart home demonstration project for individuals living with complex disabilities including intellectual impairments.

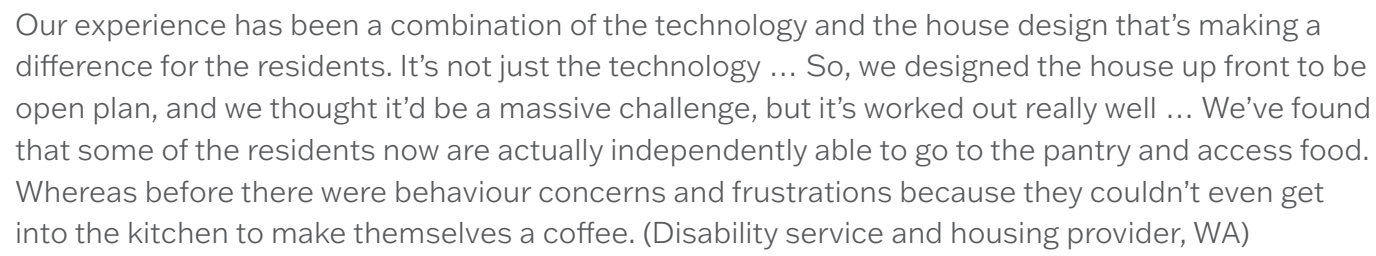

Highlighting the importance of the combination of appropriate and custom designed housing with AT, Eric's case study (below) clearly identifies the impact it has had on his ability to live as independently as possible. Well-designed housing and access to AT has given him a sense of control over his life, despite the daily need for assistance and carers in his home space. Eric's example shows how AT has changed over time; how with technological development it can quickly become 'out of date', and how the uptake or adoption of AT increasingly by the general population creates misconceptions about AT being disability approved equipment. Importantly, it demonstrates that while people may be able to financially set-up AT systems, the ongoing costs can create concern and stress unless the replacement of equipment can be funded by the NDIS. For Eric, the NDIS and SDA have been vital in enabling him to live independently despite the severity of his disability.

Navigating the NDIS system for 'reasonable and necessary' smart home technology to prolong independent living: Eric's case study

Eric is a single man in his late 50 s who has postgraduate qualifications in software, reliability, and systems engineering. He is a quadriplegic as a result of an accident in 1998. After 18 months at a Rehabilitation Centre Spinal Unit, he moved into a rented Community Housing Association wheelchair accessible home. While Eric was thankful for a home to allow independent living, the house was designed for someone with paraplegia and not suitable to his needs. He had to battle with the housing association for the house to be modified appropriately.

Over the last four years, Eric has lived independently in his own (owner designed and built) SDA registered home. He designed the home to be suitable for his needs and he believes it is a 'fantastic disability access home', though he would like to be able to complete the build to allow him access to the backyard with his wheelchair. Eric's ability to build a suitable home for his specific needs (and to remain living there) was funded using all of his savings and superannuation, loans from members of the family, and the availability of SDA funding as a part of the NDIS.

Eric needs daily help with most, if not all, of his personal tasks, but he is proud of his ability to live independently and has very strong wishes to continue to do so with as little assistance as possible. Living independently, he states implies 'he can control his environment; safely move around his house and the immediate surrounds; can maintain security and door control; and can obtain (or contact) assistance in the event of an emergency'.

Due to his physical limitations, Eric has a significant need for AT. This technology has been developed, and selffunded, over a period of years since entering his rental accommodation and then refined for his current home. At the time of design and installation, smart home devices reliant on the loT were not readily available. So his home is a cost-effective mixture of hard wired, ethernet network and Wi-Fi, PCs and servers.

Eric's smart home AT system allows him to unlock the front door remotely, control the lights, blinds, air conditioning and Wi-Fi system. While voice activation would make his life easier, he comments that at the moment there are five competing technologies and he lacks the motivation and resources to undertake a comparative analysis, so he is prepared to wait and see how these five options develop and evolve over time. Eric's computer equipment is ageing and rapidly passing its use by date despite its essentiality to his current lifestyle and wellbeing. The replacement of this equipment needs to be funded by the NDIS as his financial resources are severely constrained. However, he has had difficulties getting a Local Area Coordinator (LAC) (whose role is to assist a people to navigate 
the NDIS) to understand whether computers and associated networks are 'reasonable and necessary'. In correspondence with his LAC, Eric was informed that his computer and supporting network could not be considered AT because everyone has a computer and internet connection-backup costs of your PC, internet provider modem and Wi-Fi are all costs that an average Australian would incur regardless of their disability. In addition, he was informed that iPads are now standard household items and therefore shouldn't be covered; to argue so would require a detailed investigation. When it comes to an iPad as a specific communication or technology facilitation device, a clinical report would need to be received and it would be sent to a specialist technical team in the NDIA to review at length, with all the evidence submitted against legislation. It is quite a complex process. As Eric argues, in his case, a modified computer with voice recognition software is an essential item of daily living, and before a specialist technician team spends considerable time conducting a review, they should consider what alternative options exist to an iPad for a C3/C4 quadriplegic who lacks hand function and has all but no arm movement that would allow him to control the environment of his home and to be able to lead as 'normal' a life as possible. Despite these issues the NDIS (and SDA) is vital in supporting Eric to live as independently as possible in his own home, with AT playing an essential role.

Figure 25: Photo of Eric's DIY set-up that needs replacing with new smart home technologies

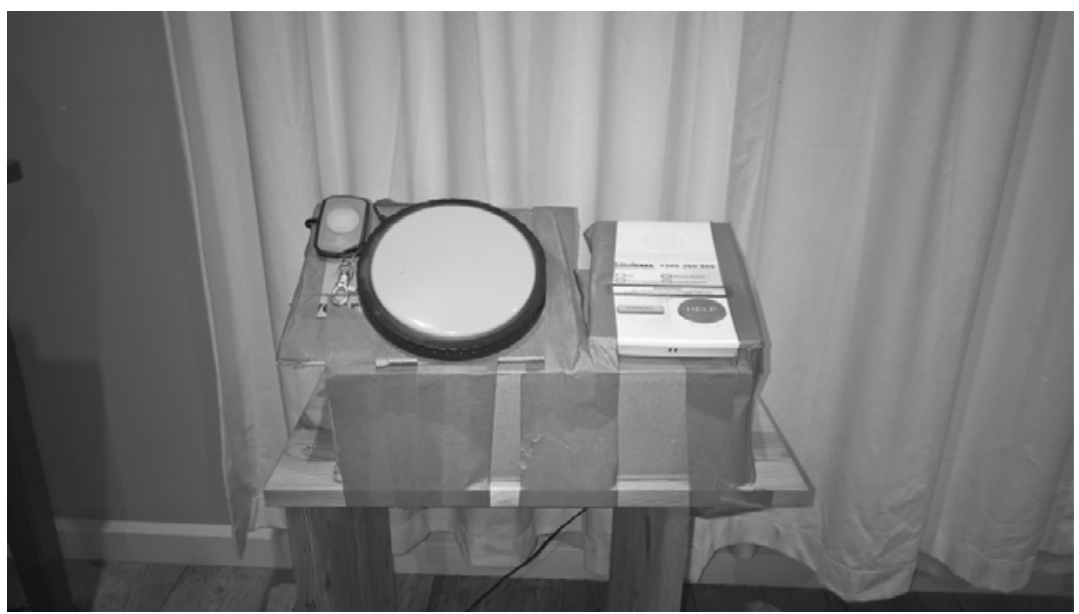

Source: Supplied by Eric.

There is a need for further research within built environment disciplines exploring the relationship between technology and housing design from the user perspective, and the implications for the design and procurement of specialist disability and aged care accommodation (Carnemolla 2018).

\subsubsection{Perceived ease of use}

Discussions around the perceived ease of use of smart home AT centred around dimensions of access, including affordability, off-the-shelf versus customised solutions, and the provision of individualised support. In the context of an expanding mainstream market, access to smart home technologies is increasing. Participants described how technology is 'now at a point where it can be right sized and right priced', with a diverse array of affordable offthe-shelf solutions that can offer a simple 'plug and play' experience.

The pros are that particularly the new emerging technology is commercially available. It's low-cost, anybody can use it, it's fairly easy to set-up. It's not a bespoke device for a person with a disability that can often cost a lot more money. (Insurance and care provider, NSW)

Taking advantage of such readily available off-the-shelf technology has created 'greater ease' with everyday tasks for Simon. Due to the availability, relatively low-cost and portability of smart home AT in the form of Google Home and Amazon Alexa, Simon was able to use such AT to his advantage. The availability of off-the-shelf AT devices means he was able to use his interest and research to purchase several products that have given him greater control over his life. 
Modifying a freestanding home to reduce care and increase autonomy after traumatic injury: Simon's case study

Simon was actively seeking to take more control of his life, day-to-day autonomy and improve his quality-of-life overall on returning home after his injury. As a result of the traumatic injuries he sustained as a young adult, Simon experiences moderate daily pain; a relatively common outcome following damage to the spinal column. His resultant severe motor difficulties restrict his mobility and ease of access about his home and within his community. Everyday limitations on his stamina and mobility stem from difficulty moving his hands and feet and breathing unassisted. Simon, who is single, lives with his mum in a freestanding home that the family owns outright in a coastal suburb in the Gold Coast region of Queensland.

Simon's smart home investment has restored some of his autonomy and independence. Just over two years ago, Simon, who self-identifies as having a long-standing interest in smart technology and computer applications, watched a documentary on TV about AT in the home, and it was this documentary that really ignited the spark to try it out in his own home. As a result of independent research, he invested in both Google Home and Amazon Alexa, which he uses to interface with his smart appliances. These include a smart TV and audio system, smart window and blind controls, air conditioning interface, security cameras and CCTV interface, alerting reminder system(s), and smart smoke detectors, which he has located in the kitchen, living room, bedroom and swimming pool area.

Simon controls his smart home set-up with his smart watch. Simon's AT system cost him several thousand dollars out of pocket, but he believes this has been worth it and he says he is 'very satisfied' with his AT system. The words he used to sum up the positives included: reduced stress and greater ease with everyday tasks not to mention greater convenience. While Simon was very satisfied with his smart home ecosystem, he did worry about the risks of power outage, and the ongoing maintenance costs and repair or parts replacement turnaround time. At present, he estimates these to be more than $\$ 200$ annually.

While Simon has friends, he says that he is often lonely. Simon's AT system has not alleviated this feeling, but it has made him feel more positive overall. For Simon, the three attributes that made him most pleased about his choices regarding his smart home set-up were its cost in relation to its effectiveness and the follow-up services he was able to get.

Figure 26: Example of smart home technology

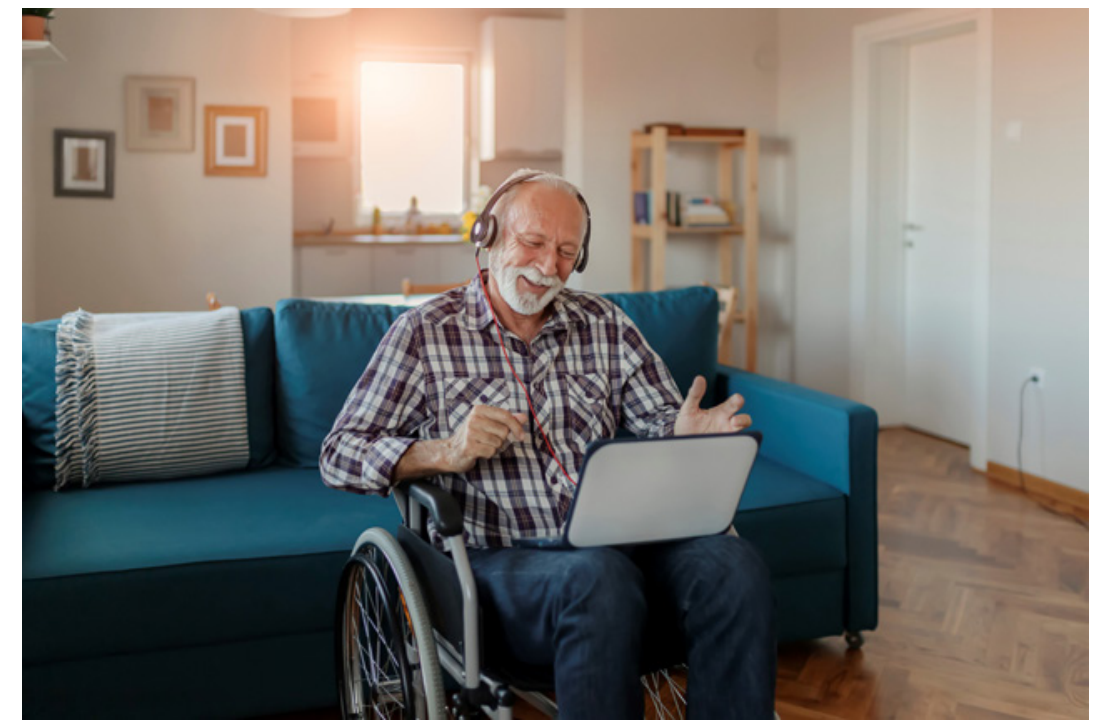

Source: purchased iStock image. 
However, the proffered out of the box 'plug and play' advantage that many smart technology products boast is not always the case.

I think for older people, it's all very complicated, understanding all these different bits that they need to have and what is Wi Fi and what's my internet plan and what's my password for all the different pieces? And there's so many little bits of the chain that can go wrong, when they don't even really quite understand what they all are. (Disability service provider, SA)

Further, for the greatest impact and effectiveness, many technologies require customisation to meet the individual needs and goals of people across a diverse range of housing circumstances. Many products in the market are developed for the mainstream. Only a very limited number include people with disability in the design process (Farahani et al. 2018) and there is limited research that evaluates the product usability of new and emerging mainstream smart home devices for people with disabilities (Layton and Steel 2019). Standard smart home technologies are being used as AT with no consideration of suitability for the end-user (Amiribesheli and Bouchachia 2018). This often requires engagement of specialists who can provide an appropriate assessment and customisation for the end-user to enable ease of use.

We don't work with just any installer. We only work with people that know how to customise the technology for people with a disability. (Insurance and care provider, NSW)

Richard's experiences with smart home technology outlined below reflects the need for assistance and support even if the product can be considered to be just 'plug and play'. Richard's case study shows the reliance of an older couple on assistance and support from a family member in setting up and maintaining various modes of AT beginning with an mCareWatch in 2012 to having portable smart home technology located throughout the home. Such technology and the comfort of knowing tech support and actual physical support is readily available has meant the difficulties of living with Richard's condition are eased.

\section{Smarter multi-family apartment ageing: Richard's case study}

Richard is a widower with severe macular degeneration, which is not uncommon; one in seven Australians over 50 years of age have some evidence of macular degeneration. Richard resides in a beach-side suburb of northern Sydney, in the state of New South Wales in an apartment building. Richard's smart technology story starts with his wife (now deceased) being diagnosed with a neurodegenerative illness a few years back. Richard's wife started wandering during both day and night and was having trouble walking. She was diagnosed with rapidly Progressive Supranuclear Palsy (PSP), which impacts cognition in a similar manner to dementia. After diagnosis, life expectancy is typically less than 10 years with good care and attention. Richard and his family, after digesting the diagnosis, decided that smart home options were a good idea. Richard's son started looking for devices that could track his mum and alert the family if she wandered off. Richard's son started with an mCareWatch in about 2012 and to help her feel okay about wearing one, Richard wore one too.

After several upgraded versions, and after the Apple Watch waterproof version was offered, Richard switched to that model because the newest watch also offered fall detection with emergency services alert. Richard, like his tech savvy son, was amenable to acquiring other smart home devices because he likes technology and because voice control allowed him to interact in many more ways as he can't read or write traditionally because of his severe macular degeneration. This led Richard, with his son's help, to decide to supplement the watch and existing iPad, with other smart home devices such as temperature sensors, CCTV, and two Google mini HomePods. Given Richard's wife's issues, they also use iPads with the Dementia clock app. The devices are located throughout their home. For instance, they have a Google mini HomePod in their living room where they watch TV and in their kitchen. Richard wears his waterproof Apple Watch in the shower and in bed at night and has an iPhone near him when the watch is charging. Most of the devices are less than two years old except their pre-existing iPads, which like many older people they were already using for reading and communicating with family prior to the onset of illness. In Richard's case though, VoiceOver describes exactly what's happening on the screen and lets you navigate using gestures or a braille display. The Display Accommodations support colour blindness and other vision needs, and Magnifier works like a digital magnifying glass-all very important with his existing level of macular degeneration. 
Richard's only son lives in the apartment directly below his parent's unit and happily set everything up for his mum and dad. Richard is comfortable and competent with their use. Although, if he accidentally changes a setting his son must figure out what he has done and take the time to fix it for him. As his son lives in the apartment directly below, this greatly reduces the barriers of sometimes frequently needing problems to be fixed. Richard and his wife have benefitted greatly from having a supportive family and the close proximity of their son who was both enthusiastic and comfortable with setting up and troubleshooting their smart device ecosystem. For instance, setting up the fall detection notifications and then ensuring the ambulance services have the necessary information to gain access into the security building and into Richard's apartment as both are electronic code-based locks. This enables Richard, and previously his wife, to continue to live independently, to be in contact with family and friends (not many now) and to access the ambulance automatically if an emergency occurs (even if his son is at work or travelling). Their smart home devices provide both peace of mind for Richard and his family, and effectively overcome most of the limitations of macular degeneration and the risk of falling and not being found.

Figure 27: Example of smart watch control interface and health monitoring

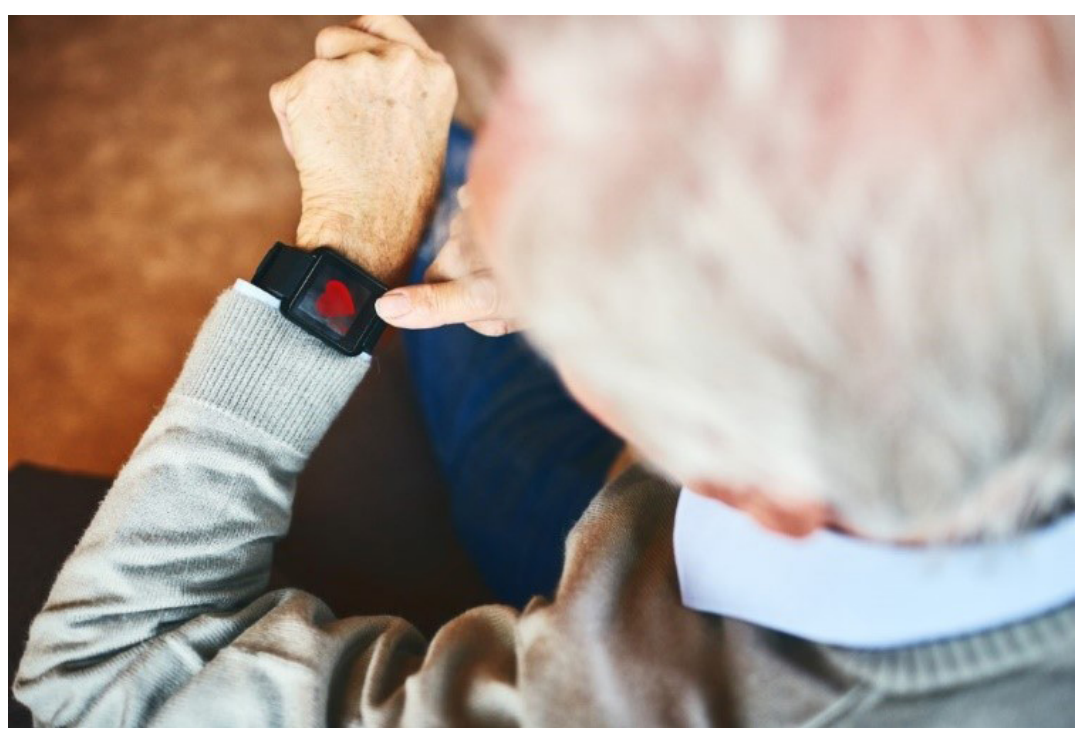

Source: Purchased iStock image.

Without customisation, smart home ATs can offer limited access for many people with both physical and intellectual impairments. One disability service and housing provider described how only 'one out of five' of their clients can interact successfully with Alexa. Initiatives such as Google's ‘Project Euphonia', where samples of speech from people with communication disabilities are collected to improve speech recognition capabilities, point to future developments that may extend access within mainstream technologies (Catania et al. 2019). However, despite technological developments and the potential for customisation, several participants raised concerns about the capacity of people who are most vulnerable, including those of advanced age and with intellectual and cognitive impairments, to effectively use smart home technologies.

Someone with a cognitive disability, either over 65 or an older person with dementia, remembering all the specific commands that Google Home is going need is a difficult task. (Aged care and housing provider, SA)

As with perceived usefulness, perceptions about ease of use are similarly predicated on the alignment of technology with the needs and abilities of individuals. A suite of initiatives is required including adaptations to technologies to reduce complexity and facilitate independent use, and individualised supports. Where individualised approaches are required, this encompasses both modifications to the technologies themselves, and supports to facilitate their use in practice, including education and training for initial implementation and ongoing technological support for repair and replacement. Of importance here is that in a consumer-directed care model, there remains a lack of clarity around who is responsible for providing that support and where the funding should come from. 
And the issue of supporting people to be able to use it is that nobody's really funded to do that. So, if you look at home care packages, or CHSP funding or whatever people are eligible for, it might fund the technology, but it doesn't really fund the advice or any training. And people are very unwilling to pay for advice... they really kind of expect that people should be offering it for free and age care just can't afford to do that anymore in the way that it's funded. (Disability service provider, SA)

Waiting for funding for AT, and what is specifically funded under aged care and NDIS packages, can be difficult for people in need to understand. While some people can make the initial outlays themselves, ongoing funding is often an issue and AT always needs upgrading at some stage. As the next case study of Mark's situation outlines, the smart home technology makes his life 'easier and safer' but he is confused and amused by what is and is not funded by the NDIS.

\section{Technology enthusiast self-funds smart home technologies: Mark's case study}

Mark is aged 53 and lives in a suburb of a small coastal city in Western Australia. He has a vision impairment and a functional neurological disorder (FND), which causes paralysis below the waist and in his left arm. This condition can come on at any time without warning and last between 12 hours to two weeks. Both Mark's FND and vision impairment have developed in the last couple of years. Mark moved into a wheelchair accessible social housing unit following his diagnosis.

To support his needs with his vision impairment and temporary paralysis, Mark uses a range of smart home assistive technologies including smart video, doorbell, security camera and CCTV, smart TV, automated lighting, heaters and fan together with a voice controlled smart phone and Google Home. He describes how this technology makes his life both easier and safer, although he described the frustrations of relying on technology during power outages and the need for backup systems.

Mark has always been interested in technology and undertook his own research on the internet to identify technologies that would meet his own needs, with some support from occupational therapists at Vision Australia and VisAbility WA. Due to the time delay between experiencing symptoms, getting a diagnosis and accessing NDIS support, Mark purchased all of the smart technologies himself. He also self-funds his internet package, which is fairly costly due to the high-speed reliable connection required.

Mark is now on an NDIS plan and has been able to access funding for additional technology supports, including a screen reader and wheelchair. If he had not already purchased the AT items, he believes that they would have been funded under his NDIS plan. However, he is uncertain about whether the NDIS would fund internet access. For Mark, access to funding to support the full costs of his needs is important as it is very difficult for him to finance extra costs living on a pension. He described the parallel situation with the NDIS funding his sports wheelchair, but not funding the sporting activities themselves - thus funding the equipment, but not the means to use it. 
Figure 28: Photo of Mark's Google Home set-up

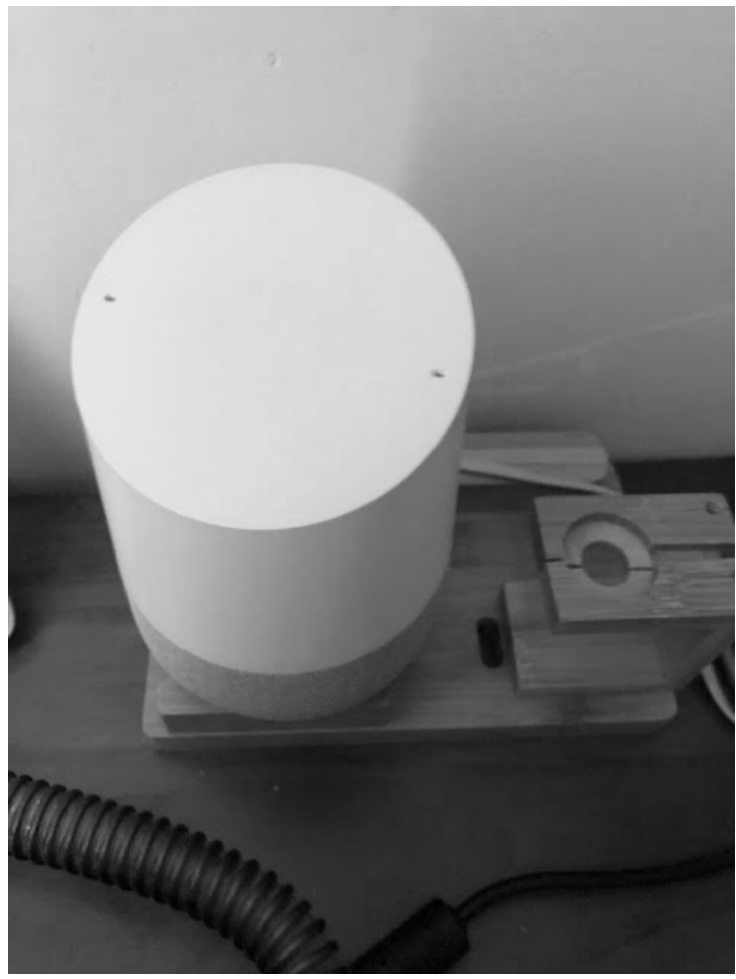

Source: Supplied by Mark

With consumer-directed care driving diversity in AT systems, there is also the risk that technologies cannot be effectively supported by carers and support workers.

... a lot of AT just never gets used ... one staff member who knows about it, but isn't there, so no one else will touch it for fear of breaking it. (Disability service and housing provider, WA)

\subsubsection{Risks and concerns}

Some participants expressed concerns about a perceived over-emphasis on smart home AT, describing it as a 'shining new toy syndrome'. Key identified risks included the protection of people who are most vulnerable, the reliability of technology, the subjective experience of care and intrusions on privacy.

While developments in smart home AT would appear to offer the greatest benefits for those with complex needs through fostering opportunities for independence that may not otherwise be attainable, there are also considerable concerns that smart home AT may actually place these people at greater risk. People living alone and those with reduced cognitive capacity were identified as being the most vulnerable. However, participants also noted the increased risks for people living in rural and remote areas where network connectivity is unreliable, reinforcing the notion of the heightened divide between different social groups (Alizadeh 2013). However, internet and power failure remain an area of potential risk for all users of AT, with examples shared of people locked in homes with smart doors during power failure with no alternative for exit or entry.

I've had people in ... the power blackout, locked in their houses because ... there was actually no key in the key safe outside. Everything's electronic. If there'd been a medical emergency, no one could have gone into the house. They would have had to smash windows ... Yeah, the concept of failure is really, really important. (Disability service provider, SA) 
More broadly, this 'concept of failure' permeated discussions about risks to all end-users. The issue of technological obsolescence, as discussed in the literature, was a central area of concern, particularly in relation to cloud-based infrastructure. However, other identified risks included failure due to cyber-attacks or user interference.

It might be that someone changes the Wi-Fi password because the kids are using it and suddenly things fall down that way. Or it may be that it's just something as simple as an account changing or part of the system falling down or no longer being supported or the cloud services no longer being available or an update. (AT advisory service, TAS).

If someone is highly reliant on that technology, like for respirators, oxygen, things like that, and if it's connected, then could it actually be a high risk because of cyber-attack? (Aged care and housing provider, NSW)

There was an articulated need for the development of government policies and regulation that stipulates minimum timeframes for maintaining cloud-based support. Managing this risk requires building appropriate redundancies into the system, and reinforces questions over whether smart home AT can reduce the need for support services.

The provision of care needs to be considered not only in its effectiveness in securing and maintaining appropriate supports, but also in relation to subjective experience (Felzmann et al. 2015). Even where risks can be managed through technological systems, participants expressed concerns about a potential over-emphasis on technology at the expense of human care.

What I get cautious about, and l'm going to put it out there and call me old fashioned or whatever, I think you can never put the relationship into technology. And what I see with people with disabilities, ageing, elderly, there is an element of vulnerability, regardless of what their condition is ... Challenging the white elephant in the room would be why do we have to keep going down this road? (Aged care and housing provider, SA)

As discussed in the literature, scepticism of the usefulness of the technology and fear of technology being used as a replacement for human care that would reduce the quality-of-care provisions is a key barrier to the adoption of new and emerging AT (Lindqvist et al. 2013). To substantiate or allay these concerns, and in the context of the increasing promotion and adoption of smart home AT, further research is required to understand the implications and impact on end-users (Martin et al. 2008).

Lastly, the risks of intrusions on privacy, both real and perceived, remain a core area of concern, including surveillance using cameras and movement tracking, data security and uncertainty and fears over who might be 'watching and listening. The risks of privacy intrusions and data breaches are one of the greatest barriers to the uptake of smart home AT (Bharucha et al. 2009; Boise et al. 2013; lenca et al. 2017). Acceptance and uptake of AT requires weighing up perceived benefits against perceived risks. As perceived usefulness of the technology increases, this can overcome privacy concerns (Courtney 2008). For the ageing population, privacy concerns are often outweighed against the ability to age in place (Townsend et al. 2011). Users also generally trade off privacy in return for convenience. However, in instances where smart home devices are being used as AT and healthcare related needs, the sensitivity of the data means the potential intrusion is much higher (Apthorpe et al. 2017) and the need of the smart home as AT is not a matter of convenience.

The complexities of navigating privacy and independence were described by one participant as a 'fine balance'. However, participants noted that not all privacy intrusions are perceived equally. Trust in care providers, demographics and individual care preferences were all seen to influence decisions in acceptance and uptake of smart home AT.

There was a view that users put greater trust in surveillance technologies offered by local providers, even if the technologies, and the surveillance, are exactly the same. 
But then you present an alarm system where you're being monitored that might be going to a call centre in a third world country. But for some reason, I don't think that's how they see it. They have the sense that it's Jackie at Head Office listening to it. So, even though they might be using exactly the same technology and have the same level of surveillance, people have more trust in the alarm that the organisation is offering them as compared to Google and things like that. (Aged care and housing provider, SA)

Younger age demographics were also seen to be more likely to accept technology due to the normalisation of technology as a part of everyday life and changing societal attitudes to data sharing. Resistance to uptake of smart home AT not only related to users of the technology, but also to older carers of family members with a disability, including both concerns about privacy and expectations about what constitutes 'care'. One participant described experiences of families removing systems and throwing them away because 'from their perspective, this is what they pay the aged care provider to do ...' (AT Provider, NSW).

This presents a picture of divergent experiences and different perceptions and attitudes to the usefulness of AT. Education has been identified as central to the uptake of smart home AT. Unless the usefulness of the AT is clearly communicated, the likelihood of adoption and use declines, and consequently negative attitudes build towards the technology (Eisma et al. 2004). Participants noted that currently in Australia there is a 'real lack of awareness' of what technologies are available and their use to support different needs in diverse settings. The overwhelming array of choice and the rapidity of development can result in a 'reluctance to go into this space' for both users of AT and advisors. There was also recognition of a need for inclusive decision-making practices early in the planning process involving negotiations between housing and service providers, the AT user and their family. There needs to be clearer communication about the relationship between smart home AT, the capacity to live independently and benefits to health and wellbeing. This was described by two participants as the potential 'tipping point' in the acceptance of smart home AT.

I think the other thing is there can be a tipping point. I don't want that in my home until you told me I can't live at home without it. (Disability service provider, SA)

I'm really excited about what [participant $x$ ] said about monitoring systems currently being reactive, which they are. But aiming towards them being proactive, that's exciting ... And could well be the tipping point in terms of people's increased acceptance of a system. (Aged and disability service provider, NSW)

\subsection{Housing and service providers}

Many housing and service providers are enthusiastic about the prospects of smart home AT and are actively introducing technology as part of their new home offerings. For example, smart home AT is being dubbed as a 'game changer' by disability housing providers such as DPN Casa Capace, who has recently opened their new specialist disability accommodation dwellings in Oran Park, Sydney (Martin 2020). The 16 new dwellings are equipped with Apple HomeKit, featuring automated blinds, adjustable benchtops and hoists. The dwellings are built to enable users to fit out more smart devices to control doors, entertainment and air conditioning from their Apple smart phone, tablet, computer or HomePod. DPN Casa Capace intends to scale this project in collaboration with Supported Independent Living (SIL) providers and suppliers as a fundamental strategy to empower people with disabilities to live more independently. Although the enthusiasm is there from housing providers, if a wider integration of smart home ATs is to occur in the disability sector, there needs to be greater support for identification and management of risk, appropriate policy and legislation, and certainty and flexibility of funding models to support participants (HOI 2012).

Participants in this study identified three key areas where this support needs to be directed: the development of robust and enduring infrastructure, advancing knowledge and innovation in the sector, and navigating the complexity of the interface between housing and care. 


\subsubsection{Infrastructure}

In the rapidly developing field of technology, questions were raised about the alignment between smart technology systems and housing in relation to longevity. Technological obsolescence, competing technologies and the rapidity of development present challenges and uncertainties in the context of identifying systems that will endure across the life of the building. This is a recognised problem for early adopters of smart home technologies, who will find themselves facing the challenges of more rapid technology obsolescence with the current market approach of built-in obsolescence (Meade and Rabelo 2004).

It's really tough because sometimes we've found we have gone into technology that two years ago was shooting the lights out, looked like it was absolutely great, had a good payback period and we thought yes, this is the one to go for. If you look at it now, you'd laugh because it's so out-of-date in comparison to what you can pick up now for half the price as well so that's the challenge that we're continuously facing. (Aged care and housing provider, NSW)

A second issue in future proofing new build housing relates to the challenges of designing infrastructure to comprehensively consider the range and diversity of needs of future residents. This requires a system design that offers flexibility at the core, enabling adaptations and additions as required to meet individual needs and circumstances.

The architectural flexibility in the system itself is quite important when it comes to preparing and ensuring the system is adaptable enough to accommodate for a wide range of needs that might be there. (AT advisory service, TAS)

System design was identified by several participants as a vital component of smart home AT. Smart home AT needs to be considered holistically as an integrated system rather than as a series of discrete elements. This is particularly challenging in the current situation with technologies working across different, and incompatible, platforms.

Most important actually is the different technologies, if there is a seamless integration between them ... If you are Google, you have to use a lot of Google compatible products, but if you have an Apple at home, then how do you make two of them work together? That sort of stuff. So, this kind of thing that is still not mature at this stage. (Housing developer, SA)

Intelligent system design is essential to ensuring longevity of smart home AT, to manage the challenges of the integration of technologies across different platforms and to meet the diverse needs of future residents in new build housing. Participants also noted particular challenges in larger homes and multiple occupancy settings to maintain internet connectivity and operability of complex systems to meet the needs of different residents. This can necessitate hardwiring and development of separate systems to support different needs within a single dwelling.

In addition to developing a systems approach, there is a need to integrate backup systems for safety for people who are most vulnerable and for those with complex needs. This can add substantially to the potential cost of the system.

... you need to have a backup option and we certainly haven't used anything that connects to the internet as a primary access method for anything that's high risk like door control. (Insurance and care provider, NSW)

... the interactions between different parts of the smart home system ... we definitely noticed that was actually one of the major costs when it comes to building, and also working with some of the clients around these systems. (AT advisory service, TAS)

Participants noted the increased costs of integrated, intelligent and flexible smart home AT systems beyond the 'basic smart home stuff'. These significant upfront costs present challenges in securing funding, including substantive lead times between construction and access to funding, uncertainties over what will be covered, and difficulties in group home settings requiring coordination across multiple packages. 
SDA is paid once the building has been certified and registered with the commission. There's a lead time of two years, where families, individuals are investing their own money, or an investor comes in and case manages alongside them. So, what we're finding is families don't actually know what they're going to be eligible for, you know. So, we sit there, and think can we get you on the NDIS plan and can you get that on the NDIS plan? And so, we're actually finding that incredibly confusing both for families, but also for a builder. (Housing developer, SA)

In that group setting ... a lot of it might be shared technology ... It can be a challenge because someone will get their funding, but you can't actually proceed until the other people get theirs, and then there are delays and that can be an issue. (Disability service and housing provider, WA)

As well as upfront costs, there is also a variety of hidden costs that are not accounted for in funding structures. AT is typically viewed as a one-off investment in hardware or software. However, in addition to upfront costs, there is a variety of operational costs. These include the costs of integrating technologies as part of a whole system (as discussed above), as well as costs of updating systems due to technological obsolescence. Several participants discussed difficulties in NDIS funding, which typically supports the initial purchase of smart home AT, but does not support associated costs of integration, or the purchase of updated technology.

There remain uncertainties over who is responsible for these costs. Technology solutions that provide upgradeable options are desirable for providers and some providers opt into a three-year contract with organisations to provide upgrades and maintenance packages.

\subsubsection{Knowledge and innovation}

Education and training are essential for advisors to ensure that appropriate technology is identified in alignment with the needs, capabilities and circumstances of individuals. Participants articulated a perceived burden of responsibility on advisors to 'do the right thing' where the user's knowledge and awareness of their needs and capabilities may be limited. Many participants spoke about the importance of education and training for occupational therapists and other service providers to facilitate uptake of smart technology.

There is a recognised lack of expertise, experience and resources for planners to understand and provide tailored solutions for individual needs (Laragy 2018). This was identified as a key gap by participants; there is a need to develop means of upskilling and dissemination of information, including knowledge of available technology, opportunities for customisation, consideration of system integration, and management of risks, such as safety and privacy provisions.

I think the issue we've found, particularly working with people with complex needs, is the lack of skilled providers who know how to assess this, how to set it up, how to customise it, how to train the staff, make sure that they've considered the safety and privacy risks and the backup systems and all of that. There's not a lot of skilled providers out there when you're working with people very complex needs, who can take all that into consideration. (Housing provider, NSW)

The challenge really, particularly for OTs working in the field of home modifications, is picking up on or identifying the right type of smart technology that suits the client's needs as well as the environment and then building in what modifications to the environment that are required. (Aged and disability service provider, NSW)

While equipment suppliers have detailed knowledge of their own technology and systems, this was seen to be narrow in scope and there was an expressed need for an independent national education model to enable effective information sharing. This was seen to be constrained by a lack of funding to develop the education model itself, as well as insufficient funding and support to encourage service providers to upskill and to effectively implement knowledge in practice. 
From an education perspective, there is no education model in this country for healthcare OTs, healthcare professionals, equipment suppliers ... It's all done by particular brands being able to run training courses... There is no funding of education at all. Our association is in the process of trying to fund it and trying to get it off the ground. We need funding to do it. (Aged care and housing provider, NSW)

... with the way billable time is for service provision, particularly under the NDIS, for example, that's a challenge to the profession in terms of keeping on top of their knowledge base and even confidence in jumping into this field and intervening and then having sufficient time to work it through ... there is a lot of work involved in terms of getting them on board in the first place. Then there's resourcing the right pieces of kit. Then there's getting approval for it if you're looking at accessing funded programmes for provision. And then there's the necessary follow up ... (Aged and disability service provider, NSW)

Education was identified as a major barrier to the advancement of smart home AT, since the lack of knowledge can 'strike fear' in providers and lead to further uncertainty for users 'who are already feeling a bit wobbly about it'. While some organisations are looking at mechanisms to integrate training and capacity building within funded projects, there needs to be greater support at government level, including funding and policies, to advance knowledge and foster innovation in smart home AT.

There is a need to think ahead - what is coming in terms of technology- are we ready, are we planning for it? ... NDA had a conference three or four years ago, promoting and looking at IT intelligence and coordination and all sorts of things. But they're not funding any of that. (Disability service provider, SA)

There is no roadmap ... You've got to burn money, waste money doing it. [Providers] need policies that's going to give them some comfort and assurance that the investment they make in their business is not going to be something that they will regret five years down the track when government changes or when something else happens and then government backtracks or changes funding models. (AT Provider, NSW)

\subsubsection{Interface between housing and care}

The third key issue in perceived enablers and barriers to the adoption of smart home AT for housing and care providers is the interface between housing and care, and the lack of clarity around responsibilities for funding of infrastructure, maintenance and ongoing supports. Both housing providers and service providers spoke about the complexity and uncertainty over who should have oversight and responsibility over different parts of the technology system, from hard wiring to home automation, maintenance and upgrades.

... working out who takes responsibility for what is important ... we are supporting clients so we provide support services, but we don't provide home automation and we don't provide a service to maintain necessarily. (Disability service provider, SA)

I think I'll just raise an interesting situation for housing providers, and the separation of housing and support and exactly where our responsibility would be and to what extent we need to have oversight over the technology that's being supplied for support. (Housing provider, NSW)

Clarifying the scope of responsibilities is even more complex in the rental market, in a context of vague legislation and tight cost margins. 
We are responsible to maintain our homes to a vague reasonable standard under the legislation. And so, if there is this technology, what is our obligation to ongoing maintenance? What's our obligation to actually install it in the first place? And then what are the cost implications for us? Obviously, we're collecting income-based rent. So, we're really working at a very, very fine margin. So, the cost consideration is major for us, looking at the longer term. (Housing provider, NSW)

From a legislative perspective, the use of mainstream smart home loT devices as medical support also presents new challenges in liability. Under the Therapeutic Goods Act this has additional onuses on businesses to manage the software changes, cyber security and other challenges that are overreached with legislation.

\footnotetext{
... the minute you have got a device ... if it's aiding and supporting someone with a disability or it can be deemed a medical device, and then under that medical device requirements, then there is additional onuses on how you actually manage, do software changes, cyber security, a whole raft of implications. And I know that it's a challenge because it could be potentially overreached with legislation. (Aged care and housing provider, NSW)
}

There was a recognised need for policies that provide a clear separation between the provision of infrastructure and its use. However, the increasing capacity of technology to provide medical support at home also raises questions about regulations and standards that guide the design and construction of the dwelling itself.

So the point you raise, I guess, about the medical devices and the TGA is very important because typically the housing stuff is operating using things like standards and building codes. And that doesn't reflect the move, if you like, of the health industry into residential accommodation outside of the hospital. (Housing provider, NSW)

\subsection{Ethics}

Ethical issues are recognised as a central concern in the adoption of smart home AT. If ethical concerns of end-users and their care givers are not adequately addressed, this often leads to scepticism and distrust of the technology (lenca et al. 2018). Key issues include the implications of smart home AT for the provision of care, including the risks of abuse and restricted practices, intrusions on privacy through surveillance and data security, and the difficulties of securing informed consent. These issues are discussed below, together with a third issue identified by participants related to equity in access to AT.

\subsubsection{Care}

The virtual assistants that are seamlessly integrated into the smart speakers enable users to execute controls of other smart devices through voice activated commands or smart interface controls. However, the virtual assistant does much more than just communicate with other smart devices in a person's home. They are sophisticated Artificial Intelligence machines that are able to learn user preferences and behavioural patterns to provide user and contextually aware prompts and information (Ehsani et al. 2017). The ability to have user and context-aware virtual assistants can be incredibly beneficial for people with disabilities and the older population. These more context aware systems have the ability to offer more individualised and tailored responses, prompts and suggestions that enhance the ease of use and convenience of everyday tasks (Chan et al. 2009). They also provide streamlined and automated care and medical support enabling independent living that may otherwise require a move to supported accommodation. Further, these technologies have the capacity for enhanced care through proactive rather than reactive supports, providing early and timely intervention and improved health outcomes.

... if we can get to a point where we could be more proactive and actually intervene a bit earlier ... not just the reactive but the ability to be ahead of the curve just that little bit ... (AT Provider, NSW) 
However, as discussed above, as smart home technologies become increasingly promoted and adopted as an affordable AT solution for ageing in place and improving the quality-of-life for people with disabilities, considerations of how this will impact formal face-to-face care provisions need to be addressed (lenca et al. 2018). Elger (2019) highlights the potential encouragement of unethical trends and isolation of participants that can occur where incentives are provided for technologies to replace human care models that can reinforce social bonds and a system of mutual care and support in society.

Further, as these technologies provide care providers and family members with increased abilities to restrict access, seclude participants and modify behaviour discretely, this raises ethical issues of restricted practices (Elger 2019). Participants questioned the boundaries between support and restrictive practices.

The other thing that's come up with our particular project is where some of this technology is restricted practice. So, if you're using, say, a GPS watch, that has a set geofencing zone, that then sets an alert that's stopping someone, or you have a sensor on the door, that lets you know that someone's got out the door and then it stops you leaving the house or other sorts of things, is that considered a restricted practice? And what kind of safeguarding do you need around that? (Housing provider, NSW)

There was an expressed need for more robust legislation and policies that are able to grapple with the grey areas on what is beneficial and increases independence, versus what is unethical restriction of freedom of movement.

You really rely on robust regulations to make sure that people are doing the right things and that you got the right people making decisions. But that's not happening which creates huge risk. (Housing provider, NSW)

In the context of the continued trajectory of the health and aged care industry to provide care at home, the lack of policy and legislation to address the intersection of care and housing is also a key concern. This raises questions about the boundaries and extent of responsibilities for care provision.

That information comes through 24 by seven. And so, providers are now looking at, well, what's the liability? What's my liability if I don't respond to a message out of hours? (AT Provider, NSW)

So, someone's got a system in there that's monitoring and then nobody responds to an alert ... And if there's no family member that's monitoring that information that's coming in, who's going to monitor that and manage that and have the duty of care? We certainly, as a funder, don't want to. There has to be some other care provider or third-party call system provider or whatever that can monitor that. (Insurance and care provider, NSW)

A few housing providers are using an opt in, opt out option to grapple with this issue of care and housing. Another participant suggested that the capacity to provide technology-augmented care at home may require a complete reconceptualisation of business models in the care sector.

... from a provider perspective ... they need to almost retool their end-to-end business to have a technology-augmented care delivery model. (AT Provider, NSW)

\subsubsection{Data privacy}

The capacity of smart home AT to constantly monitor fine-grained details of users' activities and behaviours raises ethical concerns over the protection of data privacy. This can reveal sensitive and identifiable information on user activities, including video recordings, and medical records even when they are encrypted. This puts privacy at risk (Apthorpe et al. 2017; Bugeja et al. 2016). However, there are also benefits in collecting and storing data to support evidenced-based decision making. The insights generated from the ambient monitoring dataset provides a valuable asset for care providers to understand the care needs of users and tailor care provision packages. 
Creation of an individual health dataset may increase patient autonomy whilst improving clinical treatments. However, it raises practical and legislative challenges in privacy protection, liability and security for care providers (Hodge et al. 1999). This information is also often transmitted between care providers, user, family and other stakeholders, heightening the security risks and vulnerabilities to hackers if protection protocols are not in place (Lin and Bergmann 2016). The transmission of data between organisations and in-house data management requires stringent standards to protect the privacy of users (Townsend et al. 2011). Care and housing providers need to be equipped to manage the risks of smart home AT with policies that address both the practical and ethical challenges.

The issue of data privacy was raised by many participants, including the scope of data collected by the technology itself, including where, how and how long the data is stored, who has access to it, and how the data is used. Uncertainties over ownership of data held in cloud-based infrastructure and the need to address retention laws where health data is held for substantive periods of time were identified as particular points of concern.

Service providers described conflicts between the benefits of accessing information to improve care and the need to establish clear lines of separation to protect privacy, as well as the difficulties in maintaining data security through other mechanisms, whether held by third-parties or individual users.

\begin{abstract}
... obviously we'll want access to information that we do identify for that pilot so we can see what's successful. But longer term, if we're implementing technology in the houses, we don't want to be the ones having access to that data. We'll want to have some sort of third-party provider ... But obviously we'd have concerns about how they're using that, how they're monitoring it. (Insurance and care provider, NSW)
\end{abstract}

We have tried in the past to give clients their own details and then not keeping a copy, but invariably things go missing. And we've had several where we've had to start all over again and start from scratch. (Disability service provider, TAS)

Due to the rapid nature of technology developments, there are many emergent start-up companies in this space. Providers must be extremely diligent in ensuring any company they deal with complies with data privacy laws.

I review every contract that comes in the organisation. And that's one big thing that we have is where the data has been retained. Does it comply with all the laws? If it doesn't, don't deal with that company. And also, we then won't release any data to them that's identifiable, or that we deem as something that would be intrusive. (Aged care and housing provider, NSW)

But then how is that company also using the data that they're collecting? Because all of these companies now, some of them are selling stuff that's pretty low-cost ... The value of that dataset that they are collecting, and how that can be used is the next thing. (Insurance and care provider, NSW)

Participants identified a need for improvements in legislation on data privacy and security to prevent abuse of data as well as clarity on data management procedures for service providers.

The risk of data privacy, security and abuse of vulnerable groups is a big concern when it comes to smart home IoT devices with always-on monitoring features (Wangmo et al. 2019). There is an ethical and legal responsibility to protect the users' personal privacy from unconsented surveillance (Sifford and Bharucha 2010). Often users are unaware of how loT devices operate and are, therefore, unaware of the potential data privacy intrusions and security risks associated with use (Bugeja et al. 2016). This can include transcripts of user interactions with third-party apps being retained even after they have been deleted by the user (Kelly and Statt 2019), and the outsourcing of transcriptions to third-party companies reporting confidential information, such as bank details and patient and care provider conversations (Hern 2019). This raises the issue of informed consent, which is the most frequently cited ethical concern for people with cognitive or psychologically related disabilities (Mahoney et al. 2007). Many participants voiced concern about this issue, arguing that there are currently no robust procedures for managing informed consent. 
So, whether clients are communicated clearly around the risk and implications ... I believe there is still a lot of work that the industry can do around that area. (AT advisory service, TAS)

There is a need for additional research to understand required safeguarding measures for vulnerable populations for smart home products developed for mass consumer markets. As loT devices become more pervasive and are increasingly adopted as a part of smart home AT, policy around data privacy and security needs to be introduced to protect the most vulnerable groups of society.

\subsubsection{Equity of access to AT}

From a user perspective, a third key ethical issue concerns equity in access to smart home AT. This includes the limitations of infrastructure and support in rural and regional areas, capacity for low-income households to pay for associated services such as the internet, and disparities in funding support.

In rural and regional areas, accessing smart home AT is constrained by several factors including internet reliability and the availability of both technical and professional supports.

Consistent access to reliable internet can be quite challenging. And ... having ... reliable and local access to support when things aren't working can be quite a challenge. (Disability service and housing provider, TAS)

... paying for travel time for therapists, such as OTs, to actually come out to home to assess our home to then help us actually set things up is really challenging and really expensive ... And also, in Tasmania too, I think our knowledge base in terms of having access to therapists and to providers who are really experienced in this area is a lot more limited than on the mainland. (Disability service and housing provider, TAS)

There was an articulated need for additional funding for people living in rural and regional areas to access necessary supports.

For those living in community housing or on lower incomes versus more affluent demographics, the challenge begins with having access to the internet before purchasing any smart home products. Housing providers supply accommodation, not services. In some instances, housing providers recognise difficulties of their client's experience and are in a position to provide services at a reduced cost by leveraging their bulk purchasing power. This service provision is then extended to providing smart home technology packages at a discounted cost.

Within our community housing projects and affordable housing projects, we're actually looking at doing it as part of development. So, we'd be the actual provider. And that gives us buying power for the rest of our group. (Aged care and housing provider, NSW)

In relation to funding, as the emerging technologies intersect with housing, often home modifications are required. Under the current NDIS and aged care legislation, social and community housing providers are, by default, excluded from home modification funding.

I think there's actually ... This is just brought up in terms of the accessing the system, because I know within the NDIS legislation, and within the age care legislation, there's rules around home modifications and that federal funding can't be used to fill state budgets. And so social housing and community housing by default is excluded from home modification funding from the age care system and from the NDIS as well. So that creates a big issue for us because there's no way we can actually receive funding to provide these sorts of things. (Housing provider, NSW) 
To compound the challenge, what is deemed to be infrastructure and what is personal requires clearer definitions. Across different sectors, what is perceived as infrastructure and provided accordingly may not be the case in others. For example, in aged care facilities, low tech AT such as handrails are included in packages; in retirement living, handrails could be a personal cost. Clearer definitions will facilitate the funding process and provide greater equity in access to AT.

Consultation with end-users of smart home AT and with service and housing providers has identified how valuable easy to use smart home devices such as Google Home can be at enhancing a person's wellbeing and independence at home. As a rapidly developing field, however, there is a range of current and arising issues that have policy implications. Further research is needed to gather information on the processes for the identification of appropriate technology in alignment with needs to the purchase, set-up, use and ongoing support that is required. Often people with disabilities need a more nuanced approach than what can be provided by off-the-shelf AT products, and the increased costs of integrated, intelligent and flexible smart home AT systems beyond the "basic smart home stuff' was noted. In particular, the significant lag times between planning, uncertainties over what is covered by aged care and NDIS packages and securing funding can mean users are out of pocket in the first instance or housing developments are delayed.

In addition to the initial cost outlays, there are always ongoing operational and replacement costs that are not accounted for in funding structures. With the adoption of smart technology by the 'general population', there is confusion and misconception over what are essential items to support independence.

Service providers and planners felt that there was a lack of expertise, experience and resources for assessors and planners to understand and provide tailored solutions for individual needs. This is unsurprising as this knowledge draws on knowledge of available technology, opportunities for system customisation and integration as well as the more intangible issues such as managing risks and privacy issues. This research found while some agencies were prepared to integrate training and capacity building within their organisations there needs to be greater support at all levels of government, impacting funding and policies, to advance knowledge and foster innovation in smart home AT.

Confusion exists within the marketplace regarding responsibility in the interface between housing and care. While the responsibility for the funding of infrastructure may be clear, both housing providers and service providers spoke about the complexity and uncertainty over who should have oversight and responsibility for different parts of the technology system, from hard wiring to home automation, maintenance and upgrades. While smart home AT is a valuable and promising, relatively low-cost and 'relatively easy' and an easy to install means of assistance for people to enhance and permit independent living at home. Key concerns remain unresolved about the risks of abuse and restricted practices and intrusions on privacy through surveillance and data security, as well as the difficulties of securing informed consent. Further, equity of access for a range of population groups was also identified an issue of concern.

\subsection{Policy development implications}

The case studies illustrate how the experience of end-users differs in terms of their motivation, knowledge, skill, social-support and financial capacities for AT. This means that policy and funding cannot deploy a 'one size fits all' approach to promoting and funding smart home AT. Meaningful change in servicing older people and people with disabilities is not just about changes to how funding is provided and the conditions under which people can apply but requires a dedicated and sustainable investment in education and training for sustained uptake and effective use of smart home AT. Both industry and education policy changes are needed to ensure that smart home AT is considered as a holistic system, including structures, incentives and monitoring of the relationship between technology, housing design and care. This is particularly important as different players are often exposed to only one aspect in our current siloed service and education systems. Further, the inequities in access to AT encompass geographic and socio-economic dimensions, as well as inconsistency and uncertainty in funding across different funding schemes and jurisdictions. These inequities need to be balanced with frameworks and policy structures that safeguard quality and mitigate risks (both perceived and real). 


\section{Policy development options}

This research report addresses the implications of new and emerging AT for older people and those living with a disability by focusing on the social and economic benefits and opportunities of smart home AT in supporting independent living and ageing at home. AT and home modification policy are emerging as a response to growing pressure by government funding agencies and non-government organisations advocating for older people and people with disabilities who want greater autonomy and to age in place in their local communities. However, this is a complex space. The technology itself is rapidly evolving and current guidance and policy is, at best, confusing and, at worst, absent.

A complicating factor is that smart home AT is understood to be many things by a wide variety of stakeholders, which limits its potential. Historically, AT has been derived from medical, and rehabilitative discourses. It is these discourses that have shaped much of our existing policy and practice outcomes. Consequently, the language, terminology, scope, research priorities and outcome measures have been constrained. More recently the human rights-based model now views AT as a form of prosthetic that is a human right, a need and an enabler in all aspects of the life of the person with functional impairment, including within the home where most ADL and IADL activities are carried out. Thus, how AT and smart homes are defined, and by whom, is critical. This historical background also raises questions about the role of housing designers, providers and individuals with functional limitations in their knowledge and skill, as well as the lack of an evidence-base to support more informed decision making.

Our research highlights the gaps, weakness and limitations in these policy areas. As with all research, this research raises further questions around how policymakers and government guidance on smart home technologies can improve and facilitate what look to be promising improvements in quality-of-life, care reduction and the economics of care for those with functional limitations associated with ageing or the onset of disability.

\subsection{What are the implications of new and emerging AT for older people and those living with disability?}

This research set out to explore the existing and emerging directions in the development of AT globally and nationally. However, there are issues associated with the idea that one system will fit most. Additionally, the speed of technology development has outstripped the policy and oversight mechanisms, leaving ethics issues unanswered and maintenance unclear. These issues are compounded by the speed at which technology obsolescence increases in an unstable market. In attempting to identify the current policy directions in disability and aged care in Australia and internationally for AT, it will be critical to collect data. This data must not focus on just direct costs, but also on the less tangible costs gained from closely monitoring a range of potential interrelated cost savings across mental health, relationships and care. The literature revealed that this remains problematic due to the limited number of studies investigating the end-user perspective; most studies are focused on engineering and technical capacity. Smart home AT is complex and individualistic and relies on the ability of devices to be context-aware and communicate with one another. The data logging and exchange generated from smart home AT presents concerns for privacy, security and ethics that may prevent uptake or create negative outcomes for the most vulnerable. Thus, smart home AT solutions need to appropriately take into consideration the implications for an individual should their power fail, or if their smart home AT systems are hacked or exploited. 
The factors that enable or constrain the use of AT to support independent living in practice (noted in the user survey, cost analysis, focus groups and case studies) demonstrated that there was a high satisfaction overall with most smart home AT acquired by our respondents. However, satisfaction was higher for AT Devices, and lower for Services. Our findings also echo earlier, more general AT research which found that an absence of adequate assistance impinged on quality-of-life; there is likely a substantial proportion of people whose needs remain unmet. Many of our respondents are self-funders, and there appears to be a potential gender bias, with more males being comfortable with new technology.

Notably, the case studies demonstrate how individuals who are ageing and/or who have significant functional impairments differ in terms of their motivation, knowledge, skill, social support and financial capacities. Opportunities exist where technology, housing design and care can be considered holistically. There is a variety of funding gaps related to upfront and operational costs for technology and associated infrastructure, and uncertainties over what will be covered and by whom. A further key funding gap relates to education and training for assessors and housing and care providers. Many of the focus group participants expressed the view that there needs to be greater support at the State and Commonwealth government level, including funding and policies, to advance knowledge and foster innovation in smart home AT. This could include an independent national education model and a specialist advisory group that can coordinate the necessary agencies responsible for policy and practice.

The potential economic and social benefits of policies and practices to support the development and adoption of AT in Australia remain somewhat murky. Our economic modelling indicates that smart home AT, including relatively low-cost products, supports improved outcomes and potentially contributes to cost-effective home care interventions. The survey results aided in establishing an average smart home AT cost over five years at around $\$ 700$ to $\$ 800$ with characteristic high variation ranging from above $\$ 1,000$ to below $\$ 600$. The analysis indicates improved quality-of-life outcomes, with average smart home AT costings over five years ranging from $\$ 600$ to $\$ 1,000$. However, better articulation of smart home AT product profiles targeted for defined eligibility could improve targeting of funding and servicing whilst maximising smart home AT net benefit into the future.

\subsubsection{How do new and emerging AT for older people and those living with disability interact with policy and practice?}

The development of policy in smart housing AT is important to provide an overarching vision and outline resourcing priorities. The significance of smart home AT in a person's life is evident for those who have adopted these systems. Yet, there is a lack of clear transparent pathways, protocols and policy for provision to meet individual needs. As stated by MacLachlan et al. (2018: 456) 'Assistive technology policy cuts across all sectors and ages, and it is paramount that policy initiatives recognise and reflect this'. As this research has indicated, there are potential economic and quality-of-life satisfaction gains that can be leveraged by State and Commonwealth government, the residential housing industry, smart home suppliers and end-users including older people, those with disabilities and their carers.

Australian policy in this area is fragmented and incapable of delivering at the population level. Service support for bespoke arrangements is also lacking, as illustrated in our case studies. The digital revolution is already well underway, and the planning for and investment in evidence-based guidance should be a key policy initiative. Policies must be reviewed in relation to AT for older people and those living with disability to increase uptake and improve outcomes, whilst also avoiding the risk of a wider digital and technological divide.

The lack of any national oversight is problematic; the creation of a body capable of providing oversight to ensure sustainable, efficient and effective monitoring, and supply and servicing of smart home AT is critical. However, any policy body tasked with oversight should be fully inclusive of people with a variety of smart home AT needs. This presents policymakers with the significant challenge of providing a fully integrated inclusive whole of system review of funding, tax arrangements, guidance and regulation across all housing, health, disability and telecommunication programs and initiatives. National AT policy should be charged with specifying exactly how the fractured policy space can be better informed by evidence and stimulate innovation, flexibility and shared learnings. Greater policy coordination is needed to better align the responsibilities of differing statutory and regulating bodies in the ownership and procurement, funding and coordination of services, training and information provision. 


\section{Practice}

The focus groups and case studies revealed that there is a lack of awareness, fragmented, contradictory or nonexistent service and policy standards, and a sector-wide lack of collaboration and associated sharing of research and good practice. The results from the QUEST part of the user survey indicated that there were issues with service satisfaction. The lack of supply and product and service standards are likely contributing factors in this. Additional challenges are lack of consumer-driven demand, service-related issues associated with assessment and funding, and repair and maintenance in an environment where the technology is constantly evolving.

Practitioners who participated in the focus groups have stated that they are looking for greater clarity and ongoing education to support competency development in this area. Pooling resources through innovative funding, such as co-financing, should be investigated. This may decrease the existing fragmentation of resources available whilst facilitating demand. On the supply-side, if practitioners were willing to share their knowledge, specifications and preferred product profiles, this would increase market transparency collaboration. It may also strengthen procurement for context-appropriate smart home AT whilst decreasing fragmentation.

The lack of collaboration, guidance and evidence is a major barrier to fostering a climate of innovation and efficiency that can maximise the cost gains and data gathered to build better evidence-based practice guidelines. The practitioners we interviewed understood that transformational change also meant addressing the market and better understanding the supply and demand-side barriers such as costs and who pays. However, this needs to be supported by broad consultation designed to maximise existing knowledge, increase awareness, strengthen systems and improve service delivery outcomes. Accomplishing this requires a cross-sector partnership acting as a catalyst for change. Importantly, practitioners need to actively generate policy proposals that are technically, economically and politically feasible, grounded within the language and value system practised by the policymakers. A national round table discussion on the way forward may be a good step in this direction.

\subsection{Concluding remarks}

This research finds that smart home AT and associated home modifications have the potential to improve qualityof-life for those with functional impairments in terms of care, ability to age in place, reduced institutionalisation and improved ADL and IADLs. In Australia, smart home AT is considered an integral part of health and rehabilitative care. Smart home AT has been historically prescribed, distributed, and financed through the health and disability systems without much reflection on the impact on housing design, housing expectations or access to high quality broadband.

Many levels of government are responsible for implementing housing, telecommunication and smart home AT relevant policies. Critically, legislation, regulation, guidance and funding are often State and Territory Government issues. These issues cannot be dealt with in isolation by the Australian Government, which stimulates innovation through collaboration with the states and territories through the National Housing Infrastructure Facility and provides the majority of funding for the community housing sector. Nevertheless, the Australian Government has little control of housing provision outside of its role in health and disability policy development and its shared funding arrangements. As such, there is a need for more engagement with the State and Territory Governments if more significant innovation is to be fostered.

In the future, the main arena for service and care expansion will be home-based care given that the proportion of older people and those living with chronic and multiple functional impairments is predicted to double in the next 20 years, and an associated dependency ratio is predicted to reach 58 per cent by 2042 (ABS 2018). This, in turn, will lead to increasing demand for smart home AT and home modifications. New smart home AT products need to be developed, and services concerning their delivery need to be improved, creating new possibilities and a driver for greater 'decentralisation' of skills and services to reduce and or supplement traditional care models and a need to increase available funding. This exploratory work has exposed a digital divide underscored by greater uptake by those residential aged care providers that can market their housing advantageously and individuals who can self-fund. For this to change, there is a pressing need for intersectoral collaboration resulting in greater clarity, leading to a cohesive and more mature smart home AT policy framework that promotes appropriate implementation outcomes and economic efficiencies. 


\section{References}

Alam, M. R., Reaz, M, B. R. and Ali, M. A. M. (2012) 'A Review of Smart Homes-Past, Present, and Future', IEEE Transactions on Systems, Man, and Cybernetics, Part C (Applications and Reviews), Institute of Electrical and Electronics Engineers (IEEE), 1190-1203, doi: 10.1109/tsmcc.2012.2189204.

Alizadeh, T. (2013) 'Towards the socio-economic patterns of the national broadband network rollout in Australia', State of Australian Cities Conference: Refereed Proceedings, SOAC Research Network, Sydney.

Allen, P. J. and Roberts, L. D. (2010) 'The ethics of outsourcing online survey research', International Journal of Technoethics (IJT), vol. 1, no. 3: 35-48.

National Aged Care Alliance (2018) Position Paper: Assistive Technology for Older Australians, accessed 1 April 2019 , https://naca.asn.au/wp-content/uploads/2018/11/NACA_Assistive_Technology_for_Older_Australians_Position_ Paper-1-June-2018.pdf.

Amiribesheli, M., Benmansour, A. and Bouchachia, A, (2015) 'A review of smart homes in healthcare', Journal of Ambient Intelligence and Humanized Computing, vol. 6, no. 4: 495-517, doi: 10.1007/s12652-015-0270-2.

Amiribesheli, M. and Bouchachia, H. (2018) 'A tailored smart home for dementia care', Journal of Ambient Intelligence and Humanized Computing, vol. 9, no. 6: 1755-1782, doi: 10.1007/s12652-017-0645-7.

Andrich, R., Mathiassenm, N. E., Hoogerwerf, E. J., and Gelderblom, G. J. (2013) 'Service delivery systems for assistive technology in Europe: An AAATE/EASTIN position paper', Technology and Disability, vol. 25: 127-146, doi: 10.3233/ TAD-130381.

Apthorpe, N., Reisman, D., and Feamsteer, N. (2017) A smart home is no castle: Privacy vulnerabilities of encrypted iot traffic, accessed 1 April 2019, https://arXiv preprint arXiv:1705.06805

AQoL (2014) AQoL instruments measure health-related Quality of Life, accessed 20 April 2019, https://www.agol.com.au/.

Arksey, H. and O'Malley, L. (2005) 'Scoping studies: towards a methodological framework', International Journal of Social Research Methodology, vol. 8, no. 1: 19-32, doi: 10.1080/1364557032000119616.

Ashraf, A., Liu, J. H., and Rauf, Q. (2020) 'Aging Population Perception and Post Adoption Behavior About the Usability of Smart Home Technology of Pakistani Culture', Proceedings of 2020 the 6th International Conference on Computing and Data Engineering, 2020, ACM, doi: 10.1145/3379247.3379248.

Assistive Technologies Australia (2020) What is Assistive Technology, accessed 13 September 2020, https://at-aust.org/ home/assistive_technology/assistive_technology.

Australian Bureau of Statistics (2012) '3303.0 Causes of Death, Australia', Underlying cause of death, All causes, New South Wales, accessed 14 May 2019, Australian Government, Canberra.

Australian Bureau of Statistics (2018) Population aged over 85 to double in the next 25 years, Australian Govenrment, Canberra, accessed 14 April 2020,https://www.abs.gov.au/ausstats/abs@.nsf/mediareleasesbytitle/58FF5A2527DDD 70ECA2568A90013634F.

Australian Bureau of Statistics (2018a) 4430.0 - Disability, Ageing and Carers, Australia: Summary of Findings, Australian Government, Canberra, accessed 14 April 2020, https://www.abs.gov.au/ausstats/abs@.nsf/Lookup/4430.0maint features202015. 
Australian Bureau of Statistics (2018b) Disability, Ageing and Careers, Australia, cat. no.4430.0, Australian Government, Canberra, accessed 9 March 2020, https://www.abs.gov.au/statistics/health/disability/disability-ageing-and-carersaustralia-summary-findings/latest-release.

Australian Institute of Health and Welfare (2018) Older Australia at a glance, Cat. no. AGE 87. Canberra: AlHW.

Australian Government (2018), Budget 2018-19 Budget Measures Paper no.2, accessed 20 August 2020, https://archive. budget.gov.au/2018-19/additional/budget_overview.pdf.

Australian Institute for Health and Welfare (2019) Housing assistance in Australia 2019, Cat. no. HOU 315, Canberra, accessed 21 March 2020, https://www.aihw.gov.au/reports/housing-assistance/housing-assistance-in-australia2019/contents/summary.

Barreca, S. L. (2000), Technology life-cycles and technological obsolescence, BCRI Inc., Birmingham, accesed 21 March 2020, http://www.bcri.com/Downloads/Valuation\%20Paper.PDF

Becker, H., Robert, G., Morrison, J., and Silver, J. (2004) 'Recruiting people with disabilities as research participants: Challenges and strategies to address them', Mental Retardation, vol. 42, no. 6: 471-475.

Berenguer, A., Goncalves, J., Hosio, S., Ferreira, D., Anagnostopoulos, T. and Kostakos, V.. (2016) 'Are Smartphones Ubiquitous?: An in-depth survey of smartphone adoption by seniors', IEEE Consumer Electronics Magazine, vol. 6, no. 1: 104-110.

Bethlehem, J. (2010) 'Selection bias in web surveys', International Statistical Review, vol. 78, no. 2: 161-188.

Bharucha, A. J., Anand, W., Forlizzi, J., Dew, M.A., Reynolds III, C. F., Stevens, S., and Wactlar, H. (2009) 'Intelligent assistive technology applications to dementia care: current capabilities, limitations, and future challenges', The American Journal of Geriatric Psychiatry, vol. 17, no. 2: 88-104.

Bierhoff, I., van Beerlo A., Abascal J., Allen B., Civit A., Fellbaum K., Kemppainen E., Bitterman N., Freitas D., and Kristiansson K. (2007) 'Impact and Wider Potential of Information and Communication Technologies', in Roe, P. R. W. (eds), Towards an Inclusive Future, East Sussex Press, Brussels, Belgium: 110-147

Biernacki, P. and Waldorf, D. (1981) 'Snowball Sampling: Problems and Techniques of Chain Referral Sampling', Sociological Methods \& Research, vol. 10, no. 2: 141-163, doi: 10.1177/004912418101000205.

Boise, L., Wild, K., Mattek, N., Ryhl, M., Didge, H. H., and Kaye, J. (2013) 'Willingness of older adults to share data and privacy concerns after exposure to unobtrusive in-home monitoring', Gerontechnology: International Journal on the Fundamental Aspects of Technology to Serve the Ageing Cociety, vol. 11, no. 3: 428.

Bowes A, Dawson, A., and Greasley-Adams, C. (2013) 'Literature review: the cost-effectiveness of assistive technology in supporting people with dementia', Report to the Dementia Services Development Trust, School of Applied Social Science, University of Stirling.

Brandt, A., Samuelsson, K., Töytäri, O., and Salminen, A. L(2011) 'Activity and participation, quality of life and user satisfaction outcomes of environmental control systems and smart home technology: a systematic review', Disability and Rehabilitation: Assistive Technology, vol. 6, no. 3: 189-206, doi: 10.3109/17483107.2010.532286.

Bricknell, S. (2003) 'Disability: the use of aids and the role of the environment', Disability Series, Australian Institute for Health and Welfare, Cat. No. DIS, 32.

Bridge, C., Maalsen, S., Zmudzki, F., O'Neil, S., Camemolla, P. (2016) 'DIY home modifications: What information is required at point-of-sale?', Home Modification Information Clearinghouse, vol., doi: 10.26288/5c468a878a53c.

Bridge, C., Phibbs, P., Kendig, H. L., Matthews, M. R., and Cooper, B (2006) The costs and benefits of using private housing as the 'home base' for care for older people: a systematic review, AHURI Final Report No. 94 , Australian Housing and Urban Research Institute Limited, Melbourne, https://www.ahuri.edu.au/research/position-papers/94.

Brodin, H. and Persson, J. (1995) 'Cost-Utility Analysis of Assistive Technologies in the European Commission's Tide Program', International Journal of Technology Assessment in Health Care, vol. 11, no. 2: 276-283, doi: 10.1017/S0266462300006899.

Brophy, L., Bruxner, A., and Wilson, E. (2014) 'Consumer choices about mental health support services', New paradigm, vol., no. Summer 2014: 27-29.

Brostow, D. P., Hirsch, A. T., and Kurzer, M. S. (2015) 'Recruiting older patients with peripheral arterial disease: evaluating challenges and strategies', Patient Preference and Adherence, vol. 9: 1121. 
Bugeja, J., Jacobsson, A., and Davidsson, P. (2016) 'On Privacy and Security Challenges in Smart Connected Homes', 2016 European Intelligence and Security Informatics Conference (EISIC), 2016, IEEE, doi: 10.1109/eisic.2016.044.

Calveley, J. (2012) 'Including adults with intellectual disabilities who lack capacity to consent in research', Nursing Ethics, vol. 19, no. 4: 558-567, doi: 10.1177/0969733011426818.

Carnemolla, P. (2018) 'Ageing in place and the internet of things - how smart home technologies, the built environment and caregiving intersect', Visualization in Engineering, vol. 6, no. 1:1-16, doi: 10.1186/s40327-018-0066-5.

Carnemolla, P. and Bridge, C. (2016a) 'Accessible housing and health-related quality of life: Measurements of wellbeing outcomes following home modifications', International Journal of Architectural Research: ArchNet-IJAR, vol. 10: 38-51, doi: 10.26687/archnet-ijar.v10i2.977.

Carnemolla, P. and Bridge, C. (2016b) 'Accessible housing and health-related quality of life: measurements of wellbeing outcomes following home modifications', International Journal of Architectural Research, vol. 10, no. 2: 14, doi: 10.26687/archnet-ijar.v10i2.977.

Carnemolla, P. and Bridge, C. (2019) 'Housing design and community care: How home modifications reduce care needs of older people and people with disability', International Journal of Environmental Research and Public Health, vol. 16, no. 11: 1951.

Carpenter, T., Pogacar, R., Pullig, C., Kouril, M., Aguilar, S., LaBouff, J., Isenberg, N. and Chakroff, A. (2018) 'Survey-software implicit association tests: a methodological and empirical analysis', Behavior Research Methods, vol. 51.5: 2194-2208.

Catania, F., Crovari, P., Spitale, M. and Garzotto, F. (2019) 'Automatic Speech Recognition: Do Emotions Matter?', 2019 IEEE International Conference on Conversational Data \& Knowledge Engineering (CDKE), IEEE, 9-16.

Chan, M., Campo, E. and Estève, D. (2009) 'Smart homes - Current features and future perspectives', Maturitas, vol. 64, no. 2: 90-97, doi: 10.1016/j.maturitas.2009.07.014.

Chandler, K., Willmott, L. and White, B. (2014) 'Rethinking restrictive practices: a comparative analysis', QUT Law Review, vol. 14, no. 2: 90-122.

Colombo, F. and Murakami, Y. (2013) Finland - A Good Life in Old Age, OECD, European Commission.

Condon, D. (2019) 'Heath passport to improve disability care,' Irish Health, accessed 28 March 2020, http://www. irishhealth.com/article.html?id=26706.

Corey, K. L. McCurry, M. K., Sethares, K. A., Bourbonniere, M., Hirschman, K. B., and Meghani, S. H. (2018) 'Utilizing Internet-based recruitment and data collection to access different age groups of former family caregivers', Applied Nursing Research, vol. 44: 82-87.

Courtney, K. L. (2008) 'Privacy and senior willingness to adopt smart home information technology in residential care facilities', Methods of Information in Medicine, vol. 47, no. 01: 76-81, doi: 10.3414/me9104.

Crist, R. (2020) 'Philips Hue is killing off support for the original Hue Bridge', CNET, accessed 08 March 2020, https:// www.cnet.com/news/philips-hue-is-killing-off-support-for-the-original-hue-bridge/.

Dailey, D. L., Vance, C.G., Rakel, B.A., Zimmerman, M.B., Embree, J., Merriwether, E.N., Geasland, K.M., Chimenti, R., Williams, J.M., Golchha, M. and Crofford, L.J. (2019) 'A Randomized Controlled Trial of TENS for Movement-Evoked Pain in Women with Fibromyalgia', Arthritis \& Rheumatology, vol.72, no.5: 824.

Davis, F. D. (1989) 'Perceived Usefulness, Perceived Ease of Use, and User Acceptance of Information Technology', MIS Quarterly, vol. 13, no. 3: 319-340, doi: 10.2307/249008.

De Witte, L., Steel, E., Gupta, S., Ramos, V.D. and Roentgen, U. (2018) 'Assistive technology provision: towards an international framework for assuring availability and accessibility of affordable high-quality assistive technology', Disability and Rehabilitation: Assistive Technology, vol. 13, no. 5: 467-472, doi: 10.1080/17483107.2018.1470264.

Demers, L., Weiss-Lambrou, R. and Ska, B. (2002) 'The Quebec User Evaluation of Satisfaction with Assistive Technology (QUEST 2.0): an overview and recent progress', Technology and Disability, vol. 14, no. 3: 101-105.

Department of Communities, Child Safety and Disability Services (2012) Research to inform service delivery models that include the application of smart assistive technology, South Australia, accessed 10 March 2019, http:// satcommunity.com.au/upload/117a01569fed62b6eb18fa1d024f1768.pdf. 
Department of Health, (2006) Our health, our care, our say: A new direction for community services, National Health Scheme, United Kingdom, accessed 10 March 2019, https://assets.publishing.service.gov.uk/government/uploads/ system/uploads/attachment_data/file/272238/6737.pdf.

Department of Health (2019) Commonwealth Home Support Programme, Program Manual 2018-2020, page 8, accessed 12 Feburary 2020, https://www.health.gov.au/resources/publications/commonwealth-home-supportprogramme-chsp-manual.

Department of Health (2020) Protecting older Australians: COVID-19 update 7 May 2020, Australian Government Department of Health, accessed 28 May 2020, https://www.health.gov.au/news/newsletters/protecting-olderaustralians-covid-19-update-7-may-2020.

Department of Social Sciences (2019) Review of the National Disability Insurance Scheme Act 2013, accessed 1 Feburary 2020, https://www.dss.gov.au/disability-and-carers-programs-services-for-people-with-disability-national-disabilityinsurance-scheme/review-of-the-ndis-act-report.

Dewsbury, G., Taylor, B. and Edge, M. (2001) 'Designing safe smart home systems for vulnerable people', Dependability and Healthcare Informatics Workshop, 65-70.

Disabilities Information Provision, Provision of Assistive Technology in the Nordic Countries accessed 21 Feburary 2020, https://www.dinf.ne.jp/doc/english/resource/nordic2.html.

Disability Federation of Ireland (2016) Assistive Technology for People with Disabilities and Older People, Enable Ireland, Ireland.

Economist (2020) Finland turns to technology to help frail people live at home, The Economist, Helsinki, accessed 20 Feburary 2020, https://www.economist.com/europe/2020/01/09/finland-turns-to-technology-to-help-frail-oldpeople-live-at-home.

Ehsani, F., Witt-Ehsani, S.M. and Rolandi, W., Nant Holdings IP LLC, 2017. Smart home automation systems and methods. U.S. Patent 9,614,690.

Eisma, R., Dickinson, A. and Goodman, J.S., Tiwari, \& Newell. (2004) ‘Early user involvement in the development of information technology-related products for older people', Universal Access in the Information Society, vol. 3, no. 2: 131-140, doi: 10.1007/s10209-004-0092-z.

Elger, B. S. (2019) 'Ethical Concerns About the Use of Assistive Technologies', Intelligent Assistive Technologies for Dementia: Clinical, Ethical, Social, and Regulatory Implications, p.147.

Faes, M., Faes, M., Van lersel, M. and Rikkert, M.O. (2007) 'Methodological issues in geriatric research', Journal of Nutrition Health and Aging, vol. 11, no. 3: 254.

Farahani, B., Firouzi, F., Chang, V., Badaroglu, M., Constant, N. and Mankodiya, K. (2018) 'Towards fog-driven loT eHealth: Promises and challenges of IoT in medicine and healthcare', Future Generation Computer Systems, vol. 78: 659-676, doi: 10.1016/j.future.2017.04.036.

Felzmann, H., Murphy, K., Casey, D. and Beyan, O. (2015) 'Robot-assisted care for elderly with dementia: is there a potential for genuine end-user empowerment?', The Emerging Policy and Ethics of Human Robot Interaction.

Forgasz, H., Tan, H., Leder, G. and McLeod, A. (2018) 'Enhancing survey participation: Facebook advertisements for recruitment in educational research', International Journal of Research \& Method in Education, vol. 41, no. 3: 257-270.

Francesca, C., Ana, L.N., Jérôme, M. and Frits, T. (2011) OECD Health Policy Studies Help Wanted? Providing and Paying for Long-term Care: providing and paying for long-term care, OECD Publishing, doi:10.1878/9789264097759-en.

Ginn, J. (2018) 'qualtRics: retrieve survey data using the Qualtrics APl', Journal of Open Source Software, vol. 3, no. 24: 690.

Goodacre, L. and Turner, G. (2005) 'An investigation of the effectiveness of the Quebec user evaluation of satisfaction with assistive technology via a postal survey', British Journal of Occupational Therapy, vol. 68, no. 2: 93-96.

Gower, V., Andrich, R., Agnoletto, A., Winkelmann, P., Lyhne, T., Rozis, R. and Thurmair, G. (2012) 'The European Assistive Technology Information portal (EASTIN): Improving usability through language technologies', International Conference on Computers for Handicapped Persons, Berlin, Heidelberg, Springer, 215-222, doi: 10.1007/978-3-642-31522-0_32.

Hagberg, L., Hermansson, L., Fredriksson, C. and Pettersson, I. (2017) 'Cost-effectiveness of powered mobility devices for elderly people with disability', Disability and Rehabilitation: Assistive Technology, vol. 12, no. 2: 115-120, doi: 10.3109/17483107.2014.1002540. 
Harvey Norman. (2019). Smart Home Automation Division. Unpublished raw data.

Hawthorne, G., Hawthorne, G., Korn, S. and Richardson, J. (2013) 'Population norms for the AQoL derived from the 2007 Australian National Survey of Mental Health and Wellbeing', Australian and New Zealand Journal of Public Health, vol. 37, no. 1: 7-16.

Hawthorne, G., Richardson, J. and Osborne, R. (1999) 'The Assessment of Quality of Life (AQoL) instrument: a psychometric measure of health-related quality of life', Quality of Life Research, vol. 8, no. 3: 209-224.

Hern, A. (2019) 'Apple contractors 'regularly hear confidential details' on Siri recordings', The Guardian, accessed 10 Feburary 2020, https://www.theguardian.com/technology/2019/jul/26/apple-contractors-regularly-hearconfidential-details-on-siri-recordings\#maincontent.

Hodge, J. J. G., Gostin, L.O. and Jacobson, P.D. (1999) ‘Legal Issues Concerning Electronic Health Information', JAMA, vol. 282, no. 15: 1466, doi: 10.1001/jama.282.15.1466.

Hokke, S., Hackworth, N.J., Bennetts, S.K., Nicholson, J.M., Keyzer, P., Lucke, J., Zion, L. and Crawford, S.B. (2020) 'Ethical considerations in using social media to engage research participants: perspectives of australian researchers and ethics committee members', Journal of Empirical Research on Human Research Ethics, vol. 15, no. 1-2: 12-27.

Hokke, S., Hackworth, N.J., Quin, N., Bennetts, S.K., Win, H.Y., Nicholson, J.M., Zion, L., Lucke, J., Keyzer, P. and Crawford, S.B. (2018) 'Ethical issues in using the internet to engage participants in family and child research: A scoping review', PLOS ONE, vol. 13, no. 9: e0204572, doi: 10.1371/journal.pone.0204572.

Holme, S. A., Kanny, E.M, Guthrie, M.R, Johnson, K.L. (1997) 'The use of environmental control units by occupational therapists in spinal cord injury and disease services', American Journal of Occupational Therapy, vol. 51, no. 1: 42-48.

Hong, A., Nam, C., Kim, S. (2020) 'What will be the possible barriers to consumers' adoption of smart home services?', Telecommunications Policy, vol. 44, no. 2: 101867.

Horevoorts, N. J., Vissers, P.A., Mols, F., Thong, M.S. and van de Poll-Franse, L.V. (2015) 'Response rates for patient-reported outcomes using web-based versus paper questionnaires: comparison of two invitational methods in older colorectal cancer patients', Journal of Medical Internet Research, vol. 17, no. 5: e111.

Horner-Johnson, W. and Bailey, D. (2013) 'Assessing understanding and obtaining consent from adults with intellectual disabilities for a health promotion study', Journal of Policy and Practice in Intellectual Disabilities, vol. 10, no. 3: 260-265.

lenca, M., Fabrice, J., Elger, B., Caon, M., Scoccia Pappagallo, A., Kressig, R.W. and Wangmo, T. (2017) 'Intelligent assistive technology for Alzheimer's disease and other dementias: a systematic review', Journal of Alzheimer's Disease, vol. 56, no. 4: 1301-1340, doi: 10.3233/jad-161037.

lenca, M., Wangmo, T., Jotterand, F., Kressig, R.W. and Elger, B. (2018) ‘Ethical design of intelligent assistive technologies for dementia: a descriptive review', Science and Engineering Ethics, vol. 24, no. 4: 1035-1055, doi: 10.1007/s11948017-9976-1.

Independent Living Centres Australia (2020) Using Assistive Technology, accesed 12 January 2020, https://ilcaustralia. org.au/Using_Assistive_Technology.

Jarling, A., Rydström, I., Ernsth-Bravell, M., Nyström, M. and Dalheim-Englund, A.C. (2018) 'Becoming a guest in your own home: home care in Sweden from the perspective of older people with multimorbidities', International Journal of Older People nursing, vol. 13, no. 3: e12194.

Judd, B., Liu, E., Easthope, H., Davy, L. and Bridge, C. (2014) Downsizing amongst older Australians, AHURI Final Report No. 214, Australian Housing and Urabn Research Institute, Melbourne, http://www.ahuri.edu.au/publications/ projects/p70687.

Kadam, R. A., Borde, S.U., Madas, S.A., Salvi, S.S. and Limaye, S.S. (2016) 'Challenges in recruitment and retention of clinical trial subjects', Perspectives in Clinical Research, vol. 7, no. 3: 137.

Kangasniemi, M., Karki, S., Colley, N. and Voutilainen, A. (2019) 'The use of robots and other automated devices in nurses' work: An integrative review', International Journal of Nursing Practice, vol. 25, no. 4: e12739.

Karayannis, N. V., Baumann, I., Sturgeon, J.A., Melloh, M. and Mackey, S.C. (2019) 'The impact of social isolation on pain interference: a longitudinal study', Annals of Behavioral Medicine, vol. 53, no. 1: 65-74.

Karunanithi, M. and Zhang, Q. (2018) 'An innovative technology to support independent living: the smarter safer homes platform', Stud Health Technol Inform, vol. 246: 102-110. 
Kavanagh, A. M., Krnjacki, L., Aitken, A., LaMontagne, A.D., Beer, A., Baker, E. and Bentley, R. (2015) 'Intersections between disability, type of impairment, gender and socio-economic disadvantage in a nationally representative sample of 33,101 working-aged Australians', Disability and Health Journal, vol. 8, no. 2: 191-199, doi: 10.1016/j. dhjo.2014.08.008.

Keglovits, M. and Stark, S. (2020) 'Home modifications to improve function and safety in the United States', Journal of Aging and Environment, vol.: 1-16.

Kelly, M. and Statt, N. (2019) 'Amazon confirms it holds on to Alexa data even if you delete audio files', The Verge, accessed 02 Feburary 2020, https://www.theverge.com/2019/7/3/20681423/amazon-alexa-echo-chris-coons-data-transcriptsrecording-privacy.

Khadka, J., Lang, C., Ratcliffe, J., Corlis, M., Wesselingh, S., Whitehead, C., and Inacio, M. (2019) 'Trends in the utilisation of aged care services in Australia, 2008-2016', BMC Geriatrics, vol. 19, no. 1: 213, doi: 10.1186/s12877-019-1209-9.

Khan, H. T. (2019) 'Population ageing in a globalized world: Risks and dilemmas?', Journal of Evaluation in Clinical Practice, vol. 25, no. 5: 754-760.

Khosravi, P. and Ghapanchi, A. H. (2016) 'Investigating the effectiveness of technologies applied to assist seniors: A systematic literature review', International Journal of Medical Informatics, vol. 85, no. 1: 17-26, doi: 10.1016/j.ijmedinf. 2015.05.014.

Kinsella, B. (2019) Australia Leaps Past U.S. in Smart Speaker Adoption, Google Home Establishes Dominant Market Share [Online], voicebot.ai, accessed 20 Feburary 2020, https://voicebot.ai/2019/03/19/australia-leaps-past-u-s-insmart-speaker-adoption-google-home-establishes-dominant-market-share/.

Kohli-Lynch, C. N. and Briggs, A. H. (2019) Heterogeneity in Cost-Effectiveness Analysis, Oxford Research Encyclopedia of Economics and Finance, doi: 9780190625979.013 .101

Kolodinsky, J., Cranwell, M. and Rowe, E. (2002) 'Bridging the generation gap across the digital divide: Teens teaching internet skills to senior citizens', Training, vol. 5, no. 10: 2 .

Lansley, P. (2004) 'Can adapting the homes of older people and providing assistive technology pay its way?', Age and Ageing, vol. 33, no. 6: 571-576, doi: 10.1093/ageing/afh190.

Laragy, C. (2018) 'The NDIS is delivering 'reasonable and necessary' supports for some, but others are missing out', The Conversation, 1 October, accessed 1 Feburary 2020, https://theconversation.com/the-ndis-is-deliveringreasonable-and-necessary-supports-for-some-but-others-are-missing-out-97922.

Larsson Ranada, A. and Lidström, H. (2019) 'Satisfaction with assistive technology device in relation to the service delivery process-A systematic review', Assistive Technology, vol. 31, no. 2: 82-97.

Lau, J., Zimmerman, B. and Schaub, F. (2018) 'Alexa, Are You Listening?', Proceedings of the ACM on Human-Computer Interaction, vol. 2, no. CSCW: 1-31, doi: 10.1145/3274371.

Layton, N. and Steel, E. (2019) 'The convergence and mainstreaming of integrated home technologies for people with disability', Societies, vol. 9, no. 4: 69, doi: 10.3390/soc9040069.

Leahy, A. (2018) 'Paradoxically thinking: ageing with disability, disability with ageing', International Network for Critical Gerontology, accessed 10 March 2019, https://criticalgerontology.com/paradoxically-ageing-disability/.

Lee, A. (2019) 'Australian loT@home market cracks \$1bn, paving the way for loT-commerce services', Telsyte, accessed 29 January 2020, https://www.telsyte.com.au/announcements/2019/5/14/australian-iothome-market-cracks-1bnpaving-the-way-for-iot-commerce-services.

Lennox, N., Taylor, M., Rey-Conde, T., Bain, C., Purdie, D.M. and Boyle, F. (2005) 'Beating the barriers: recruitment of people with intellectual disability to participate in research', Journal of Intellectual Disability Research, vol. 49, no. 4: 296-305.

LERA (2019) The State of The Smart Home Market, LERA Smart Home Solutions, accessed 13 May 2020, https:// lerasmarthomes.com.au/smart-home-market/.

Li, J., Han, X., Zhang, X. and Wang, S. (2019) 'Spatiotemporal evolution of global population ageing from 1960 to 2017', BMC Public Health, vol. 19, no. 1: 127.

Lin, H. and Bergmann, N. (2016) 'IoT Privacy and Security Challenges for Smart Home Environments', Information, vol. 7, no. 3: 44, doi: 10.3390/info7030044. 
Lindqvist, E., Nygård, L. and Borell, L. (2013) 'Significant junctures on the way towards becoming a user of assistive technology in Alzheimer's disease', Scandinavian Journal of Occupational Therapy, vol. 20, no. 5: 386-396, doi: 10.3109/11038128.2013.766761.

Liu, L., Stroulia, E., Nikolaidis, I., Miguel-Cruz, A. and Rincon, A.R. (2016) 'Smart homes and home health monitoring technologies for older adults: A systematic review', International Journal of Medical Informatics, vol. 91: 44-59, doi: 10.1016/j.jijmedinf.2016.04.007.

Maalim, M., MacLachlan, M., Long, S., O’Donnell, J., Ahern, S., Gilligan, J. and Maalim, M. (2019) 'Access to assistive technology: A descriptive review and application of systems-thinking approach in the conceptualization of the assistive technology passport', Global Perspectives on Assistive Technology, vol.: 489.

Macdonald, S. J. and Clayton, J. (2013) 'Back to the future, disability and the digital divide', Disability \& Society, vol. 28, no. 5: 702-718, doi: 10.1080/09687599.2012.732538.

MacLachlan, M., Banes, D., Bell, D., Borg, J., Donnelly, B., Fembek, M., Ghosh, R., Gowran, R.J., Hannay, E., Hiscock, D. and Hoogerwerf, E.J. (2018) 'Assistive technology policy: a position paper from the first global research, innovation, and education on assistive technology (GREAT) summit', Disability and Rehabilitation: Assistive Technology, vol. 13, no. 5: 454-466.

Mahoney, D. F., Purtilo, R.B., Webbe, F.M., Alwan, M., Bharucha, A.J., Adlam, T.D., Jimison, H.B., Turner, B., Becker, S.A. and Working Group on Technology of the Alzheimer's Association (2007) 'In-home monitoring of persons with dementia: Ethical guidelines for technology research and development', Alzheimer's \& Dementia, vol. 3, no. 3: 217-226, doi: 10.1016/j.jalz.2007.04.388.

Malbon, E. and Carey, G. (2019) 'Women, rural and disadvantaged Australians may be missing out on care in the NDIS', The Conversation, 11 July, accessed 01 Feburary 2020 https://theconversation.com/women-rural-and-disadvantagedaustralians-may-be-missing-out-on-care-in-the-ndis-120081.

Malta, S. and Wilding, R. (2018) 'The digital divide: small, social programs can help get seniors online'. The Conversation, 27 August, accessed 01 Feburary 2020 https://theconversation.com/the-digital-divide-small-social-programs-canhelp-get-seniors-online-99082.

Mann, W. C. (1999) 'Effectiveness of assistive technology and environmental interventions in maintaining independence and reducing home care costs for the frail elderly: a randomized controlled trial', Archives of Family Medicine, vol. 8 , no. 3: 210-217, doi: 10.1001/archfami.8.3.210.

Markit, I. (2019) IoT Device Spotlight: Smart Speaker, accessed 16 March 2020, https://www.iotworldtoday.com/files/2019/ 10/IHS-Markit-loT-Device-Smart-Speaker-1.pdf.

Marsden, E. and Lee, P. (2016) There's no place like phone: Consumer usage patterns in the era of smartphone, Deloitte Global Mobile Consumer Survey 2016: UK cut, Deloitte Global Mobile Consumer Survey 2016, accessed 15 March 2019, http://www.deloitte.co.uk/mobileuk2016/assets/pdf/Deloitte-Mobile-Consumer-2016-There-is-no-place-likephone.pdf.

Martin, K. (2020) 'Prime Minister opens multi-dwelling disability housing project', Freedom2Live, 26 February 2020, accessed 27 Feburary 2020, https://www.freedom2live.com.au/prime-minister-opens-multi-dwelling-disabilityhousing-project/\#.XIhxHpMzasr.

Martin, S., Kelly, G., Kernohan, W.G., McCreight, B. and Nugent, C. (2008) 'Smart home technologies for health and social care support', Cochrane Database of Systematic Reviews 4.

Meade, P. T. and Rabelo, L. (2004) 'The technology adoption life cycle attractor: Understanding the dynamics of high-tech markets', Technological Forecasting \& Social Change, vol. 71, no. 7: 667-684, doi: 10.1016/j.techfore.2004.01.008.

Melkas, H. (2013) 'Innovative assistive technology in Finnish public elderly-care services: A focus on productivity', Work, vol. 46, no. 1: 77-91.

Mitzner, T. L., Savla, J., Boot, W.R., Sharit, J., Charness, N., Czaja, S.J. and Rogers, W.A. (2019) 'Technology adoption by older adults: findings from the PRISM trial', The Gerontologist, vol. 59, no. 1: 34-44.

Morris, M. E., Adair, B., Miller, K., Ozanne, E., Hansen, R., Pearce, A.J., Santamaria, N., Viega, L., Long, M. and Said, C.M. (2013) 'Smart-home technologies to assist older people to live well at home', Journal of Aging Science, vol. 1, no. 1:1-9.

National Disability Insurance Scheme (2015) Assistive Technology Strategy, National Disability Insurance Scheme, accessed 02 Feburary 2020, https://www.ndis.gov.au/media/204/download. 
National Disability Insurance Agency (2019) NDIA Assistive Technology \& Consumables Code Guide, National Disability Insurance Agency, accessed 02 Feburary 2020, https://www.ndis.gov.au/media/1461/download

National Disability Insurance Scheme (2019a) Choosing assistive technology, National Disability Insurance Scheme, accessed 04 March 2020, https://www.ndis.gov.au/participants/home-equipment-and-supports/assistivetechnology-explained/choosing-assistive-technology\#mid-cost-at.

National Disability Insurance Scheme (2019b) Specialist Disability Accomodation (SDA) Design Standard, National Disability Insurance Scheme, accessed 02 Feburary 2020, https://www.ndis.gov.au/providers/housing-and-livingsupports-and-services/housing/specialist-disability-accommodation/sda-design-standard.

Noda, K. (2018) 'Google Home: smart speaker as environmental control unit', Disability and Rehabilitation: Assistive Technology, vol. 13, no. 7: 674-675, doi: 10.1080/17483107.2017.1369589.

NSW Ageing and Disability Commission (2019) Review into Disability Advocacy in NSW, accessed 02 Feburary 2020, https://www.ageingdisabilitycommission.nsw.gov.au/_data/assets/pdf_file/0008/765530/Review-into-DisabilityAdvocacy-in-NSW.pdf.

Obaid, T., Rashed, H., El Nour, A.A., Rehan, M., Saleh, M.M. and Tarique, M. (2014) 'Zigbee Based Voice Controlled Wireless Smart Home System', International Journal of Wireless \& Mobile Networks, vol. 6, no. 1: 47-59, doi: 10.5121/ijwmn. 2014.6104.

Ocepek, J., Roberts, A.E. and Vidmar, G. (2013) 'Evaluation of treatment in the smart home IRIS in terms of functional independence and occupational performance and satisfaction', Computational and Mathematical Methods in Medicine, vol. 2013: 1-10, doi: 10.1155/2013/926858.

Olney, S. and Dickinson, H. (2019) 'Australia's new National Disability Insurance Scheme: implications for policy and practice', Policy Design and Practice, vol. 2, no. 3: 275-290, doi: 10.1080/25741292.2019.1586083.

Parette, H. P., Huer, M.B. and Scherer, M. (2004) 'Effects of acculturation on assistive technology service delivery', Journal of Special Education Technology, vol. 19, no. 2: 31-41.

Patel, M. X., Doku, V. and Tennakoon, L. (2003) 'Challenges in recruitment of research participants', Advances in Psychiatric Treatment, vol. 9, no. 3: 229-238.

Perry-Duxbury, M., van Exel, J. and Brouwer, W. (2019) 'How to value safety in economic evaluations in health care? A review of applications in different sectors', The European Journal of Health Economics, vol. 20, no. 7: 1041-1061, doi: 10.1007/ s10198-019-01076-9.

Persson, J. and Husberg, M. (2012) 'Can we rely on QALYs for assistive technologies?', Techbnology and Disability, vol. 24, no.1: 93-100

Pennsylvania Assistive Technology Foundation (PATF), Smart Homes Made Simple, accessed 14 February 2021, https:// smarthomesmadesimple.org/.

Portz, J. D., Bayliss, E.A., Bull, S., Boxer, R.S., Bekelman, D.B., Gleason, K. and Czaja, S. (2019) 'Using the technology acceptance model to explore user experience, intent to use, and use behavior of a patient portal among older adults with multiple chronic conditions: descriptive qualitative study', Journal of Medical Internet research, vol. 21, no. 4: e11604.

Priestley, M. (2002) 'Whose voices? Representing the claims of older disabled people under New Labour', Policy \& Politics, vol. 30, no. 3: 361-372.

Productivity Commission (2011a) Caring for Older Australians: Overview, Report No. 53, Final Inquiry Report, accesed 14 April 2020, https://www.pc.gov.au/inquiries/completed/aged-care/report.

Productivity Commission (2011b) Disability Care and Support: Productivity Commission Inquiry, Report No. 54, accessed 14 April 2020, https://www.pc.gov.au/inquiries/completed/disability-support/report.

Productivity Commission (2017a) Regulation in the Digital Age, Shifting the Dial: 5 year Productivity Review, Supporting Paper No. 13, accesed 18 April 2020, https://www.pc.gov.au/inquiries/completed/productivity-review/report.

Productivity Commission (2017b) Shifting the Dial: 5 Year Productivity Review, Report No. 84, accessed 18 April 2020, https://www.pc.gov.au/inquiries/completed/productivity-review/report.

Productivity Commission (2019) Report on Government Services: 15 Services for people with disability, accessed 18 April 2020, https://www.pc.gov.au/research/ongoing/report-on-government-services/2019/community-services/ services-for-people-with-disability. 
Productivity Commission (2015) Housing decisions of older Australians, accessed 14 April 2020, https://www.pc.gov.au/ research/completed/housing-decisions-older-australians/housing-decisions-older-australians.pdf

Richardson, J., Peacock, S., lezzi, A., Day, N., \& Hawthorne, G. (2007). The Assessment of Quality-of-life (AQoL) II Instrument; Overview and Creation of the Utility Scoring Algorithm. Monash University Centre for Health Economics, Research Paper 2007 (17) (https://www.monash.edu/_data/assets/pdf_file/0009/893484/respap17. pdf). doi:10.1192/bjp.bp.113.136036.

Richter, F. (2019) 'How Long Does Apple Support Older iPhone Models?', STATISTA, accessed 12 Feburary 2020, https:// www.statista.com/chart/5824/ios-iphone-compatibility/.

Rossignuolo, V. (2020) 'Is planned obsolescence for smart devices becoming obsolete?', SMS Global, 13 March 2020, accessed 16 march 2020, https://www.smsglobal.com/blog/planned-obsolescence/.

Russell, S. J., Siostrom, K., Edwards, I. and Srikanth, V. (2020) 'Consumer experiences of home care packages', Australasian Journal on Ageing, vol., doi: 10.1111/ajag.12771.

Saizmaa, T. and Kim, H.-C. (2008) 'A holistic understanding of HCl perspectives on smart home', 2008 Fourth International Conference on Networked Computing and Advanced Information Management, IEEE, 59-65.

Schäuble, D., Marian, A. and Cremonese, L. (2020) 'Conditions for a cost-effective application of smart thermostat systems in residential buildings', Applied Energy, vol. 262: 114526.

Scherer, M. J. and Glueckauf, R. (2005) 'Assessing the benefits of assistive technologies for activities and participation', Rehabilitation Psychology, vol. 50, no. 2: 132-141, doi: 10.1037/0090-5550.50.2.132.

Sifford, K. S. and Bharucha, A. (2010) 'Benefits and challenges of electronic surveillance in nursing home research', Research in Gerontological Nursing, vol. 3, no. 1: 5-10.

Simon, S. and Patrick, A. (1997) 'Understanding and assessing consumer satisfaction in rehabilitation', Journal of Rehabilitation Outcomes Measurement, vol. 1, no. 5:1-14.

Smith, A. C., Thomas, E., Snoswell, C.L., Haydon, H., Mehrotra, A., Clemensen, J. and Caffery, L.J. (2020) 'Telehealth for global emergencies: Implications for coronavirus disease 2019 (COVID-19)', Journal of Telemedicine and Telecare, vol.: 1357633X20916567.

Solomon, D. H., Shadick, N.A., Weinblatt, M.E., Frits, M., lannaccone, C., Zak, A. and Korzenik, J.R. (2017) 'Clinical patient registry recruitment and retention: a survey of patients in two chronic disease registries', BMC Medical Research Methodology, vol. 17, no. 1: 59.

Song, Y. and Van Der Cammen, T. J. M. (2019) ‘Electronic assistive technology for community-dwelling solo-living older adults: A systematic review', Maturitas, vol. 125: 50-56, doi: 10.1016/j.maturitas.2019.04.211.

Suzurikawa, J., Sawada, Y., Sakiyama, M., Suwa, M., Inoue, T., \& Kondo, T. (2021). Perspectives of Multidisciplinary Professional Teams during Assessment Processes for ATD Selection in the Japanese Public Provision System. International Journal of Environmental Research and Public Health, 18(5), 2697.

International Organisation for Standardisation (2016) ISO 9999:2016 Assistive products for perons with disability Classification and terminology, accessed 20 Jan 2019, https://www.iso.org/standard/60547.html.

Statista (2020) Global loT end-user spending worldwide 2017-2025, accessed 06 March 2020 https://www.statista.com/ statistics/976313/global-iot-market-size/.

Stefanov, D. H., Bien, Z. and Bang, W.C. (2004) 'The smart house for older persons and persons with physical disabilities: structure, technology arrangements, and perspectives', Transactions on Neural Systems and Rehabilitation Engineering, IEEE, 228-250, doi: 10.1109/tnsre.2004.828423.

Sund, T. (2017) Assistive Technology in Norway, NAV Department of Assistive Technology, accessed 20 March 2020, https://www.nav.no/_/attachment/inline/7b119b1c-fe72-488a-a1ef-be424e72faff:c52b8c6ee759299749538a6fd0554 d1efa695abf/assistive-technology-in-norway-170217v2.pdf.

Tegart, G., Harvey, E., Livingstone, A., Martin, C. and Ozanne, E. (2014) 'Assistive health technologies for independent living', Report for the Australian Council of Learned Academies.

The Economist (2020) 'Finland turns to technology to help frail old people live at home', 11 January 2020, accessed 15 April 2020 , https://www.economist.com/europe/2020/01/09/finland-turns-to-technology-to-help-frail-oldpeople-live-at-home. 
The Royal Commission into Aged Care Quality and Safety (2019) A History of Aged Care Reviews, accessed 08 October 2019, https://agedcare.royalcommission.gov.au/publications/Documents/background-paper-8.pdf.

Thompson, D., Fisher, K.R. and Kayess, R.. (2012) The Role of Assistive Technology in Supporting People with Disabilies and Complex Care Needs: A Literature Review, Social Policy Research Centre, Sydney.

Townsend, D., Knoefel, F. and Goubran, R. (2011) ‘Privacy versus autonomy: A tradeoff model for smart home monitoring technologies', 2011 Annual International Conference of the IEEE Engineering in Medicine and Biology Society, 2011, IEEE, doi: 10.1109/iembs.2011.6091176.

Wangmo, T., Lipps, M., Kressig, R.W. and lenca, M. (2019) 'Ethical concerns with the use of intelligent assistive technology: findings from a qualitative study with professional stakeholders', BMC Medical Ethics, vol. 20, no. 1, doi: 10.1186/ s12910-019-0437-z.

Watson, W., Clapperton, A. and Mitchell, R. (2011) 'The burden of fall-related injury among older persons in New South Wales', Aust N Z J Public Health, vol. 35, no. 2: 170-5, doi: 10.1111/j.1753-6405.2010.00656.x.

Wessels, R. and Witte, L. D. (2003) 'Reliability and validity of the Dutch version of QUEST 2.0 with users of various types of assistive devices', Disability and Rehabilitation, vol. 25, no. 6: 267-272.

Wilson, C. K., Thomas, J. and Barraket, J. (2019) 'Measuring digital inequality in Australia: The Australian digital inclusion index', Journal of Telecommunications and the Digital Economy, vol. 7, no. 2: 102-120.

World Health Organisation (2004) WHO Centre for Health Development Ageing and Health Technical Report, World Health Organisation, Geneva.

World Health Organisation (2015) World Report on Ageing and Health, World Health Organisation, Geneva.

World Health Organisation (2018) Innovative Social Services and Support Measures for Independent Living in Advanced Age UNECE Policy Brief on Ageing No.20, accessed 06 March 2020, https://www.unece.org/fileadmin/DAM/pau/ age/Policy_briefs/ECE-WG1-28.pdf.

World Health Organisation (2018) Integrated Care for Older People: Realigning Primary Health Care to Respond to Population ageing, World Health Organisation, Geneva.

World Health Organization (2019) Global Perspectives on Assistive Technology: Proceedings of the GReAT Consultation 2019, World Health Organisation, Geneva, 22-23 August 2019, vol. 1.

Wright, K. (1999) 'The communication of social support within an on-line community for older adults: A qualitative analysis of the SeniorNet community', Communication Quarterly, vol. 47, no. 4: S33.

Wright, K. B. (2005) 'Researching internet-based populations: Advantages and disadvantages of online survey research, online questionnaire authoring software packages, and web survey services', Journal of Computer-mediated Mediated Communication, vol. 10, no. 3: JCMC1034.

Zalunzny, P. (2019) 'How to create your own smart home: the basics', Choice, accesed 29 October 2019 https://www. choice.com.au/electronics-and-technology/gadgets/tech-gadgets/buying-guides/iot-smart-home. 


\section{Appendix 1: User survey questions}

\section{Section A - Assistive Devices}

The questions in this section are related to the assistive devices integrated in the home. Please refer to the image below on the types of assistive technologies and systems the survey refers to.

Q1. Identify what smart home assistant system you have by ticking the box below:

Google Home

Amazon Alexa

$\bigcirc$ Apple HomePod

Microsoft Cortana

Other? Please specify

Q2. Identify what control interface(s) you use by ticking the box(s) below:

$\bigcirc$ Smart Speaker - Voice Control

$\bigcirc$ Tablet interface such as iPad

$\bigcirc$ Smart Phone interface such as iPhone or Samsung

$\bigcirc$ Other? Such as one touch controls or personal adaptation, please specify

Q3. Identify what your assistive device(s) control in your home by ticking the box(s) below:

\section{Thermal}

Automated Air Conditioning

Automated Fan

$\bigcirc$ Other, please tell us

\section{Entertainment}

O Smart TV

$\bigcirc$ Smart Audio Systems

White Noise Control System

$\bigcirc$ Automated Lighting

\section{Openings}

Automated Curtain/Blind control

$\bigcirc$ Automated Window control

$\bigcirc$ Automated Door control

\section{Safety}

Security Camera / CCTV

$\bigcirc$ Alerting Reminder System(s)

$\bigcirc$ Gas Automatic Shutoff

$\bigcirc$ Electricity Automatic Shutoff

$\bigcirc$ Water main automatic shutoff

Smart Smoke Detectors

\section{Other}

$\bigcirc$ Automated track hoist facilities

Smart Appliances

$\bigcirc$ Smart Furnishings

$\bigcirc$ Assisted breathing device

Home Backup Generator

Other, please tell us 
Q4. Tell us where you have the assistive device(s) in your home by selecting the boxes below:

$\bigcirc$ Entry

$\bigcirc$ Living Room

Kitchen

$\bigcirc$ Bathroom

$\bigcirc$ Bedroom

Other - Garden / Yard

Other - Garage, Gate

$\bigcirc$ Other - Please Specify

Q5. What was the total upfront cost of the assistive device(s)?

$\mathrm{O} \$ 200$ or less

○201- $\$ 500$

\$501- $\$ 1000$

\$1001 - \$2000

\$2001 or more

Q6. Did you receive any government support for the assistive device(s)?

$\bigcirc$ Yes

$\mathrm{ONo}$

\section{Skip To: Q9 if Q.6 is a No}

Q7. Which was the main government support you received for the assistive device(s)?
$\bigcirc$ Aged Pension
$\bigcirc$ Disability Pension
$\bigcirc$ Other, Please Specify

Q8. How much government support did you receive for the assistive device(s)?
\$200 or less
$\$ 201-\$ 500$
\$501- $\$ 1000$
\$1001-\$2000
\$2001 or more

Q9. How much did you contribute out of pocket?

None

$\bigcirc 200$ or less

$\$ 201-\$ 500$

\$501- $\$ 1000$

\$1001- $\$ 2000$

\$2001 or more

Q10. In the past year, what is your estimated annual ongoing cost for the assistive device(s)?

○200 or less

$\bigcirc \$ 201-\$ 500$

$\bigcirc \$ 501-\$ 1000$

\$1001- $\$ 2000$

\$2001 or more

The following questions relate to how satisfied you are with your assistive device(s) and services. There may be aspects that are not directly applicable to your device(s), however, please answer this to the best of your ability.

You will also have the opportunity to add additional comments. This is optional.

Q11. How satisfied are you with the components of your assistive device(s)?

$\bigcirc$ Not satisfied at all

Not very satisfied

More or less satisfied

$\bigcirc$ Quite satisfied

$\bigcirc$ Very satisfied

Please upload photo(s) of your assistive device(s) - optional

Q12. How satisfied are you with the cost of your assistive device(s)?

Not satisfied at all

$\bigcirc$ Not very satisfied

$\bigcirc$ More or less satisfied

Quite satisfied

$\bigcirc$ Very satisfied 
Q13. How satisfied are you with the ease in adjusting (fixing, fastening) the parts of your assistive device(s)?

Not satisfied at all (1)

$\bigcirc$ Not very satisfied (2)

More or less satisfied (3)

$\bigcirc$ Quite satisfied (4)

Very satisfied (5)

Q14. How satisfied are you with how safe and secure your assistive device(s) is?

Not satisfied at all (1)

Not very satisfied (2)

More or less satisfied (3)

$\bigcirc$ Quite satisfied (4)

Very satisfied (5)

Q15. How satisfied are you with the durability (endurance, resistance to wear) of your assistive device(s)?

Not satisfied at all

Not very satisfied

More or less satisfied

$\bigcirc$ Quite satisfied

$\bigcirc$ Very satisfied

Q16. How satisfied are you with how easy it is to use your assistive device?

Not satisfied at al

Not very satisfied

More or less satisfied

$\bigcirc$ Quite satisfied

$\bigcirc$ Very satisfied

Q17. How satisfied are you with how comfortable your assistive device(s) is?

$\bigcirc$ Not satisfied at all

Not very satisfied

More or less satisfied

$\bigcirc$ Quite satisfied

$\bigcirc$ Very satisfied
Q18. How satisfied are you with how effective your assistive device(s) is (the degree to which your device(s) meets your needs)?

Not satisfied at all

$\bigcirc$ Not very satisfied

More or less satisfied

$\bigcirc$ Quite satisfied

$\bigcirc$ Very satisfied

Q19. How satisfied are you with the service delivery program (procedures, length of time) in which you obtained your assistive device(s)?

$\bigcirc$ Not satisfied at all

$\bigcirc$ Not very satisfied

More or less satisfied

$\bigcirc$ Quite satisfied

Very satisfied

Q20. How satisfied are you with the repairs and servicing (maintenance) provided for your assistive device(s)?

Not satisfied at all

$\bigcirc$ Not very satisfied

More or less satisfied

$\bigcirc$ Quite satisfied

Very satisfied

Q21. How satisfied are you with the quality of the professional services (information, attention) you received for using your assistive device(s)?

$\bigcirc$ Not satisfied at all

$\bigcirc$ Not very satisfied

More or less satisfied

$\bigcirc$ Quite satisfied

$\bigcirc$ Very satisfied

Q22. How satisfied are you with the follow-up services (continuing support services) received for using your assistive device(s)?

$\bigcirc$ Not satisfied at all

$\bigcirc$ Not very satisfied

More or less satisfied

$\bigcirc$ Quite satisfied

Very satisfied 
Q23. Below is the list of the same 12 satisfaction items. PLEASE SELECT THE THREE ITEMS that you consider to be the most important to you. Please tick 3 boxes.
1. Components
2. Cost
3. Adjustments
4. Safety
5. Durability
6. Easy to use
7. Comfort
8. Effectiveness
9. Service delivery
10. Repairs/servicing
11. Professional service
12. Follow-up services

\section{Section B - Quality-of-life Assessment}

The following section contains questions related to the quality-of-life before and after use of assistive device(s).

aqol1. Do you need any help looking after yourself? (For example: dressing, bathing, eating)

BEFORE use of smart home assistive device(s)

$\bigcirc$ I need no help at all.

$\bigcirc$ Occasionally I need some help with personal care tasks.

$\bigcirc$ I need help with the more difficult personal care tasks.

I need daily help with the most or all personal care tasks.

AFTER use of smart home assistive device(s)

$\bigcirc$ I need no help at all.

$\bigcirc$ Occasionally I need some help with personal care tasks.

$\bigcirc$ I need help with the more difficult personal care tasks.

$\bigcirc$ I need daily help with the most or all personal care tasks. aqol2. When doing household tasks: (For example: cooking, cleaning the house, washing).

BEFORE use of smart home assistive device(s)

$\bigcirc$ I need no help at all.

$\bigcirc$ Occasionally I need some help with household tasks.

$\bigcirc$ I need help with the more difficult household tasks.

$\bigcirc$ I need daily help with most or all household tasks.

AFTER use of smart home assistive device(s)

$\bigcirc$ I need no help at all.

Occasionally I need some help with household tasks.

$\bigcirc$ I need help with the more difficult household tasks.

$\bigcirc$ I need daily help with most or all household tasks.

aqol3. Thinking about how easily you can get around your home and community:

BEFORE use of smart home assistive device(s)

$\bigcirc$ I get around my home and community by myself without any difficulty.

I find it difficult to get around my home and community by myself.

I cannot get around the community by myself, but I can get around my home with some difficulty.

$\bigcirc$ I cannot get around either the community or my home by myself.

AFTER use of smart home assistive device(s)

$\bigcirc$ I get around my home and community by myself without any difficulty.

I find it difficult to get around my home and community by myself.

I cannot get around the community by myself, but I can get around my home with some difficulty.

$\bigcirc$ I cannot get around either the community or my home by myself. 
aqol4. Because of your health, your relationships (for example: with your friends, partner or parents) generally:

BEFORE use of smart home assistive device(s)

$\bigcirc$ Are very close and warm.

$\bigcirc$ Are sometimes close and warm.

$\bigcirc$ Are seldom close and warm.

$\bigcirc$ I have no close and warm relationships.

AFTER use of smart home assistive device(s)

$\bigcirc$ Are very close and warm.

$\bigcirc$ Are sometimes close and warm.

Are seldom close and warm.

$\bigcirc$ I have no close and warm relationships.

aqol5. Think about your relationship with other people:

BEFORE use of smart home assistive device(s)

$\bigcirc$ I have plenty of friends, and am never lonely.

Although I have friends, I am occasionally lonely.

$\bigcirc$ I have some friends, but am often lonely for company.

$\bigcirc$ I am socially isolated and feel lonely.

AFTER use of smart home assistive device(s)

$\bigcirc$ I have plenty of friends, and am never lonely.

$\bigcirc$ Although I have friends, I am occasionally lonely.

$\bigcirc$ I have some friends, but am often lonely for company.

I am socially isolated and feel lonely.

aqol6. Thinking about your health and your relationship with your family:

BEFORE use of smart home assistive device(s)

$\bigcirc$ My role in the family is unaffected by my health.

There are some parts of my family role I cannot carry out.

$\bigcirc$ There are many parts of my family role I cannot carry out.

$\bigcirc$ I cannot carry out any part of my family role.
AFTER use of smart home assistive device(s)

My role in the family is unaffected by my health.

$\bigcirc$ There are some parts of my family role I cannot carry out.

$\bigcirc$ There are many parts of my family role I cannot carry out.

$\bigcirc$ I cannot carry out any part of my family role.

aqol7. Thinking about your vision, including when using your glasses or contact lenses if needed:

BEFORE use of smart home assistive device(s)

$\bigcirc$ I see normally.

$\bigcirc$ I have some difficulty focusing on things, or I do not see them sharply. For example: small print, a newspaper or seeing objects in the distance.

$\bigcirc$ I have a lot of difficulty seeing things. My vision is blurred. For example: I can see just enough to get by.

I only see general shapes, or I am blind. For example: I need a guide to move around.

AFTER use of smart home assistive device(s)

$\bigcirc$ I see normally.

$\bigcirc$ I have some difficulty focusing on things, or I do not see them sharply. For example: small print, a newspaper or seeing objects in the distance.

$\bigcirc$ I have a lot of difficulty seeing things. My vision is blurred. For example: I can see just enough to get by.

$\bigcirc$ I only see general shapes, or I am blind. For example: I need a guide to move around.

aqol8. Thinking about your hearing, including using your hearing aid if needed:

BEFORE use of smart home assistive device(s)

$\bigcirc$ I hear normally.

$\bigcirc$ I have some difficulty hearing or I do not hear clearly. For example: I ask people to speak up, or turn up the TV or radio volume.

I have difficulty hearing things clearly. For example: Often I do not understand what is said. I usually do not take part in conversations because I cannot hear what is said.

$\bigcirc$ I hear very little indeed. For example: I cannot fully understand loud voices speaking directly to me. 
AFTER use of smart home assistive device(s)

$\bigcirc$ I hear normally.

I have some difficulty hearing or I do not hear clearly. For example: I ask people to speak up, or turn up the TV or radio volume.

$\bigcirc$ I have difficulty hearing things clearly. For example: Often I do not understand what is said. I usually do not take part in conversations because I cannot hear what is said.

$\bigcirc$ I hear very little indeed. For example: I cannot fully understand loud voices speaking directly to me.

aqol9. When you communicate with other: (For example: by talking, listening, writing or signing.)

BEFORE use of smart home assistive device(s)

$\bigcirc$ I have no trouble speaking to others or understanding what they say.

$\bigcirc$ I have some difficulty being understood by people who do not know me. I have no trouble understanding what others say to me.

$\bigcirc$ I am only understood by people who know me well. I have great trouble understanding what others say to me.

I cannot adequately communicate with others.

AFTER use of smart home assistive device(s)

I have no trouble speaking to others or understanding what they say.

$\bigcirc$ I have some difficulty being understood by people who do not know me. I have no trouble understanding what others say to me.

$\bigcirc$ I am only understood by people who know me well. I have great trouble understanding what others say to me.

I cannot adequately communicate with others.

aqol10. Thinking about how you sleep:

BEFORE use of smart home assistive device(s)

I am able to sleep without difficulty most of the time.

My sleep is interrupted some of the time, but I am usually able to go back to sleep without difficulty.

My sleep is interrupted most nights, but I am usually able to go back to sleep without difficulty.

$\bigcirc$ I sleep in short bursts only. I am awake most of the night.
AFTER use of smart home assistive device(s)

I am able to sleep without difficulty most of the time.

My sleep is interrupted some of the time, but I am usually able to go back to sleep without difficulty.

$\bigcirc$ My sleep is interrupted most nights, but I am usually able to go back to sleep without difficulty.

$\bigcirc$ I sleep in short bursts only. I am awake most of the night.

aqol11. Thinking about how you generally feel:

BEFORE use of smart home assistive device(s)

I do not feel anxious, worried or depressed.

$\bigcirc$ I am slightly anxious, worried or depressed.

$\bigcirc$ I feel moderately anxious, worried or depressed.

$\bigcirc$ I am extremely anxious, worried or depressed.

AFTER use of smart home assistive device(s)

I do not feel anxious, worried or depressed.

I am slightly anxious, worried or depressed.

$\bigcirc$ I feel moderately anxious, worried or depressed.

I lam extremely anxious, worried or depressed.

aqol12. How much pain or discomfort do you experience?

BEFORE use of smart home assistive device(s)

$\bigcirc$ None at all.

I have moderate pain.

I suffer from severe pain.

I suffer unbearable pain.

AFTER use of smart home assistive device(s)

None at all.

$\bigcirc$ I have moderate pain.

$\bigcirc$ I suffer from severe pain.

I suffer unbearable pain. 


\section{Section C - Demographic Questions}

The following section contains general demographic questions. If you are a carer or family member, please answer these questions in relation to the person who uses the assistive device(s).
Q1. What is your age group?
0 $0-17(1)$
○ $17-65(2)$
$65+(3)$

Q2. What is your gender?

Male (1)

$\bigcirc$ Female (2)

Other ( please specify) (3)

Q3. What are your functional limitation(s)? Tick one or more

A. COGNITION balance or blackouts (vestibular), disfigurement or deformity, emotional (mental), child, cognitive (intellectual, sensory, memory or learning)

B. EYES loss of vision (not corrected by glasses)

C. EARS loss of hearing (not corrected by aid)

D. MOUTH communication (loss of speech)

E. HEART/LUNG energy or stamina

F. HANDS older, reach or dexterity (incomplete use of arms or fingers and/or difficulty gripping)

$\bigcirc$ G. HANDS/LEGS incoordination or tremor or balance sensation (numbness, pain or tingling chronic or recurring), universal or no obvious impairment

H. FEET mobility (incomplete use of feet or legs)

$\bigcirc$ I. SCALES extremes of size and weight

\section{Functional Limitations}

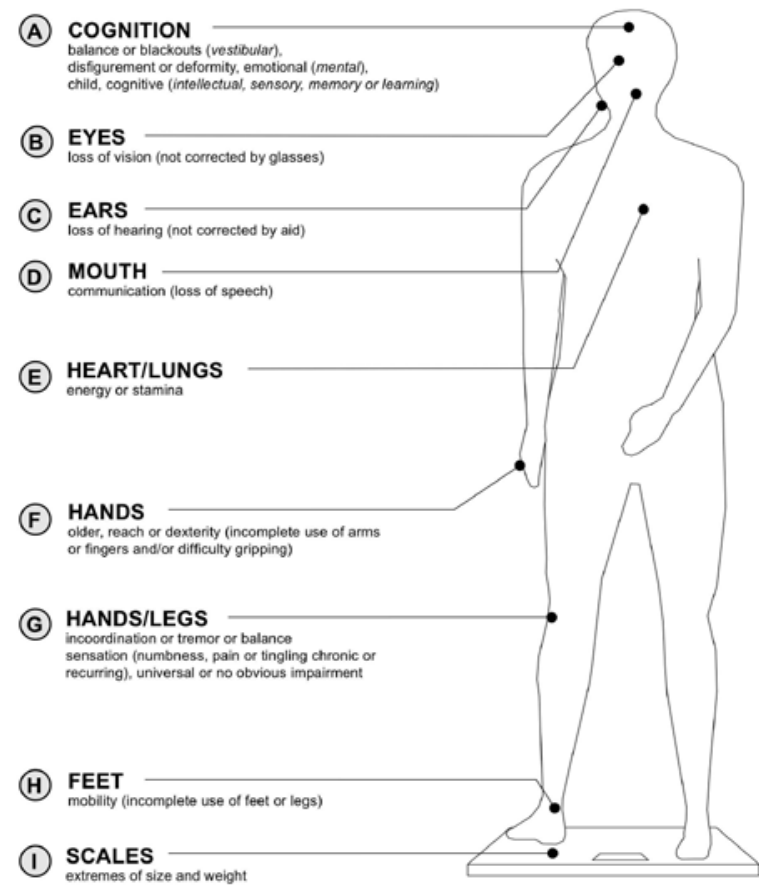

Adaptod by Bridge 2005 to use International Classification of Function constructs from the original Enabler ideogram created by Steinfeld, et al. 1979

Q4. Where were you born?

Australia

Other (please specify)

Skip To: Q6. If you were born in Australia. (Q4 = Australia)

Q5. If not born in Australia, how long have you lived here? Please enter in YEARS

Q6. What is the main language spoken in your home?

$\bigcirc$ English

Other (please specify)

Q7. What is your current marital status?

$\bigcirc$ Never married

$\bigcirc$ Divorced

$\bigcirc$ Separated but not divorced

$\bigcirc$ Widowed

Married 
Q8. What is your current accommodation?

$\bigcirc$ Owner - Outright or Mortgaged

$\bigcirc$ Rental - Private Market or Social Housing

Specialist Disability Accommodation

$\bigcirc$ With Family or Relatives

If Q8. = Owner - Outright or Mortgaged. Please Answer Q9.

Q9. Do you:

Own it outright

$\bigcirc$ Own it with a mortgage

If Q8. = Rental - Private Market o Social Housing. Please Answer Q10.

Q10. Who is your rental provider?

Private Market

$\bigcirc$ Social Housing

$\bigcirc$ Community Housing

Other (please specify)

If Q8. = With Family or Relatives. Please answer Q11.

Q11. Which of the following best describe your relationship with family/relative members in your accommodation?

Partner

Partner and Children

$\bigcirc$ Children

Other (please specify)

Q12. How many people live in your accommodation?

○ 1

$\bigcirc 2$

○ 4 - 7

○ $8-15$

Q13. Is your current accommodation a:

$\bigcirc$ Separate house

$\bigcirc$ Semi-detached, row or terrace house, townhouse

Flat, unit or apartment

$\bigcirc$ Other dwelling: (please explain)
Q14. How long have you lived in your current accommodation?

$\bigcirc$ Under 1 year

1-4 years

5-9 years

10-20 years

$\bigcirc$ Over 20 years

Q15. What is your yearly HOUSEHOLD income before tax, from all sources? Including benefits, pensions, superannuation, etc.

$\bigcirc$ Less than $\$ 5,000$ per year

\$5, 000 - \$9, 999 peryear

\$10, 000 - \$19, 999 per year

\$20, 000 -\$29, 999 per year

$\bigcirc \$ 30,000$ - \$39, 999 per year

$\bigcirc \$ 40,000$ - $\$ 49,999$ per year

$\bigcirc \$ 50,000$ - \$69, 999 per year

\$70,000 or more per year

Q16. What State/Territory of Australia do you live in? New South Wales

$\bigcirc$ Victoria

$\bigcirc$ Queensland

Western Australia

$\bigcirc$ Tasmania

Northern Territory

$\bigcirc$ Australian Capital Territory

South Australia

Q17. Please tell us who is filling in the survey?

Primary user of the assistive device(s)

Assisting one of the above persons to complete the survey

If Q17. = Primary user of the assistive device(s).

Please answer Q.18

If D14 = Primary user of the assistive device(s)

Q18. Please tell us if you are:

65 or under

66 or over 
Q19. Would you be willing to be contacted to provide further information on assistive device(s)?

$\bigcirc$ Yes

$\mathrm{No}$

If $\mathrm{Q} 19=$ Yes. Please answer the following questions.

Please give us your details so that we can contact you.

Title:

First Name:

Last Name:

Street Address:

Suburb:

State:

Postcode

Contact Number:

Email: 


\section{Appendix 2: List of organisations contacted for user survey distribution}

Contacted Organisations Distributed Survey

3Bridges Community No

Anglicare Tasmania No

Anglicare SA

Yes

ASAN No

Assistive Technology Australia

Yes

Association for Children with Disability Tas (Inc) No

Autism South Australia No

Autism Tasmania

No

Baptist Care Yes

Barkuma Community Support

Yes

Blind Citizens Australia/Tasmania No

Brain Injury Association of Tasmania No

CARA Yes

Carers Tasmania No

Centacare Yes

Cerebral Palsy Tasmania No

Community Based Support (Tasmania)

No

Community Care Tasmania No

COTA NSW Yes

COTA SA Yes

COTA Tas No

DES (Domiciliary Equipment Service) No

DirectCare Australia Yes

Down Syndrome Tasmania

No

Eyre Futures Yes

Freedom 2 Live Yes 


\begin{tabular}{|c|c|}
\hline Contacted Organisations & Distributed Survey \\
\hline Harvey Norman Smart Home Commercial & Yes \\
\hline Housing Choices Tasmania & No \\
\hline Icare & No \\
\hline Independent Living Centre Tasmania & No \\
\hline Li-Ve Tasmania & No \\
\hline Life Ability & Yes \\
\hline Lutheran Disability Services & Yes \\
\hline Masonic Care Tasmania & No \\
\hline Mosaic Tas & No \\
\hline Multicap & No \\
\hline Multiple Schllerosis Society of SA \& NT & No \\
\hline Neurological Alliance Tasmania & No \\
\hline Nexus & No \\
\hline Orana & Yes \\
\hline Paraquad Associateion of SA & Yes \\
\hline ParaQuad Tasmania & No \\
\hline Physical Disability Council of New South Wales & Yes \\
\hline Possability & No \\
\hline SCHMMS Ltd t/as Scope Access & No \\
\hline Scope & No \\
\hline SCOSA Inc & No \\
\hline South Australia Speech and Hearing Centre & Yes \\
\hline Southern Cross Care & No \\
\hline Speak Out & No \\
\hline Spinal Cord Injuries Australia & No \\
\hline St Giles & Yes \\
\hline St Michaels & No \\
\hline SummitCare Smithfield & No \\
\hline TADWA (Technology Assisting Disability Western Australia) & No \\
\hline TasDeaf (Expression Australia) & No \\
\hline Wesley Mission & No \\
\hline YoungCare & No \\
\hline Total Number of Organisations Asked & 60 \\
\hline Total Number of Organisations Agreed & 20 \\
\hline
\end{tabular}

* Organisations listed as 'yes' agreed in principle to distribute the survey via their selected channels. The agreement was either made in writing or via verbal agreement, whether the distribution actually occurred or when it occurred cannot be verified by the authors. The selected platforms, frequency of posts and format of posts was also unable to be monitored, and likewise with organisations who did not respond is being listed as 'no', could have distributed the survey without informing the authors. 


\section{Appendix 3: NDIS AT complexity table}

\begin{tabular}{|c|c|c|c|c|}
\hline Complexity level & Description and examples & $\begin{array}{l}\text { Is an assessment } \\
\text { required before I can } \\
\text { access NDIS funds? }\end{array}$ & Where can I buy this AT? & Examples of smart home \\
\hline $\begin{array}{l}\text { Level } 1 \text { - Simple, } \\
\text { low-risk AT }\end{array}$ & $\begin{array}{l}\text { Simple, low-risk products } \\
\text { and services that are easy } \\
\text { to buy and need no or very } \\
\text { little assistance to set-up } \\
\text { and use. } \\
\text { Examples: } \\
\text { - Non-slip bathmat } \\
\text { - Large print labels } \\
\text { - Doorbells. }\end{array}$ & $\begin{array}{l}\text { Not required: These are } \\
\text { the everyday AT items that } \\
\text { you can easily access and } \\
\text { test out that don't cost } \\
\text { much or require support. }\end{array}$ & $\begin{array}{l}\text { You can choose to buy } \\
\text { these from everyday } \\
\text { suppliers. } \\
\text { Examples: } \\
\text { - Your local store } \\
\text { (hardware, pharmacy } \\
\text { etc.); or } \\
\text { - Suppliers you find on } \\
\text { the internet. }\end{array}$ & $\begin{array}{l}\text { - Amazon Echo } \\
\text { - Electronic Cables } \\
\text { - Low-cost electronic } \\
\text { devices }\end{array}$ \\
\hline $\begin{array}{l}\text { Level } 2 \text { - } \\
\text { Standard AT }\end{array}$ & $\begin{array}{l}\text { AT you can buy easily } \\
\text { 'off-the-shelf', test and } \\
\text { trial before making a final } \\
\text { choice. However, you } \\
\text { might need help to set-up. } \\
\text { Examples: } \\
\text { - Bath seat } \\
\text { - Hand rails } \\
\text { - Ramp. }\end{array}$ & $\begin{array}{l}\text { May be required: } \\
\text { Depending on availability } \\
\text { and your individual } \\
\text { circumstance, you may } \\
\text { require an assessment. }\end{array}$ & $\begin{array}{l}\text { You can typically find } \\
\text { this type of AT from } \\
\text { an AT supplier. }\end{array}$ & $\begin{array}{l}\text { Applications or software } \\
\text { made specifically for } \\
\text { people who are blind } \\
\text { and don't see well. }\end{array}$ \\
\hline $\begin{array}{l}\text { Level } 3 \text { - } \\
\text { Specialised } \\
\text { AT solutions }\end{array}$ & $\begin{array}{l}\text { Similar to Level } 2 \text { AT, but } \\
\text { requires modification or is } \\
\text { tailored to suit your needs. } \\
\text { Examples: } \\
\text { - Desktop electronic } \\
\text { magnification. } \\
\text { - Pressure mattresses. }\end{array}$ & $\begin{array}{l}\text { Required: You will require } \\
\text { experienced professional } \\
\text { support to help identify } \\
\text { and set-up (and, in some } \\
\text { cases train) before you can } \\
\text { most effectively use it. }\end{array}$ & $\begin{array}{l}\text { You should work with } \\
\text { your AT assessor } \\
\text { to identify suitable } \\
\text { supplier/s from which } \\
\text { you are able to source } \\
\text { the most appropriate AT } \\
\text { solution for your needs. }\end{array}$ & $\begin{array}{l}\text { - Environmental control } \\
\text { units } \\
\text { - Specialised interfaces } \\
\text { and programming. }\end{array}$ \\
\hline $\begin{array}{l}\text { Level } 4 \text { - } \\
\text { Complex } \\
\text { AT solutions }\end{array}$ & $\begin{array}{l}\text { Custom made AT which } \\
\text { is specially made or } \\
\text { configured for you. } \\
\text { - myoelectric prosthetic } \\
\text { - cochlear } \\
\text { - implant speech } \\
\text { processors. }\end{array}$ & $\begin{array}{l}\text { Required: You will require } \\
\text { specialist and/or ongoing } \\
\text { support (including } \\
\text { specialised training) to } \\
\text { identify, buy and use. }\end{array}$ & $\begin{array}{l}\text { You should work with } \\
\text { your AT assessor } \\
\text { to identify suitable } \\
\text { supplier/s from which } \\
\text { you are able to source } \\
\text { the most appropriate AT } \\
\text { solution for your needs. } \\
\text { In some cases the NDIA } \\
\text { may indicate which } \\
\text { providers are suitable. }\end{array}$ & $\begin{array}{l}\text { - Rewiring dwelling for } \\
\text { network. } \\
\text { - Installation of fixtures. }\end{array}$ \\
\hline
\end{tabular}




\section{Appendix 4: List of invited focus group participants}

\begin{tabular}{|c|c|c|c|}
\hline Organisation Name & Type & State & Attended \\
\hline Ability Technology & Disability service provider & NSW & No \\
\hline $\mathrm{ACH}$ & Aged care and housing provider & SA & Yes (SA Focus Group) \\
\hline Achieve Australia & Disability service and housing provider & NSW & Yes (NSW Focus Group 1) \\
\hline Anglicare & $\begin{array}{l}\text { Aged and disability service and housing } \\
\text { provider }\end{array}$ & Tasmania & Yes (Online Focus Group 1) \\
\hline Assistive Technologies Australia & AT advisory service & NSW & Yes (NSW Focus Group 2) \\
\hline ATSA & Disability housing provider & NSW & No \\
\hline Baptist Care & Aged care and housing provider & SA & No \\
\hline Bridge Housing & Housing provider & NSW & No \\
\hline CARA & Disability service provider & SA & Yes (SA Focus Group) \\
\hline Cerebral Palsy AT Services & Disability service provider & NSW & No \\
\hline $\begin{array}{l}\text { CHIA (Community Housing Industry } \\
\text { Associate) }\end{array}$ & Housing provider & NSW & Yes (NSW Focus Group 2) \\
\hline Community Based Support & Aged and disability service provider & Tasmania & Yes (Online Focus Group 1) \\
\hline Community Housing & Housing provider & Tasmania & No \\
\hline COTA NSW & Statutory agency & NSW & Yes (NSW Focus Group 2) \\
\hline COTA SA & Statutory agency & SA & No \\
\hline Disabilities SA & Peak body & SA & No \\
\hline Disability Support Network & Peak body & SA & No \\
\hline Domiciliary Care & Disability service provider & SA & No \\
\hline $\mathrm{ECH}$ & Aged care and housing provider & SA & Yes (SA Focus Group) \\
\hline Enable NSW & Disability service provider & NSW & No \\
\hline Enable NSW Home & Disability housing provider & NSW & No \\
\hline Enlighten & Disability service provider & NSW & No \\
\hline Fresh Hope Care & Aged care and housing provider & NSW & Yes (NSW Focus Group 2) \\
\hline Harvey Norman & Technology Supplier & NSW & No \\
\hline Homestay & AT Provider & NSW & Yes (Online Focus Group 2) \\
\hline Housing Choices & Housing provider & Tasmania & No \\
\hline
\end{tabular}




\begin{tabular}{|c|c|c|c|}
\hline Organisation Name & Type & State & Attended \\
\hline Housing Tasmania & Statutory agency & Tasmania & No \\
\hline Hume Housing & Housing provider & NSW & Yes (NSW Focus Group 1) \\
\hline iCare & Insurance and care provider & NSW & Yes (NSW Focus Group 2) \\
\hline Independent Living Centre & AT advisory service & Tasmania & $\begin{array}{l}\text { Yes (Online Focus Group } 1 \\
\text { and 2) }\end{array}$ \\
\hline Independent Living Centre & AT advisory service & SA & No \\
\hline IRT Group & Aged care and housing provider & NSW & Yes (NSW Focus Group 1) \\
\hline Land and Housing Cooperation & Statutory agency & NSW & No \\
\hline Li-Ve & Disability service and housing provider & Tasmania & Yes (Online Focus Group 2) \\
\hline Lifetime Homes & Housing provider & Tasmania & No \\
\hline Mosaic & Disability service provider & Tasmania & No \\
\hline Multicap & Disability service and housing provider & Tasmania & No \\
\hline Multiple Sclerosis Society of SA and NT & Peak body & SA & No \\
\hline My Aged Care & Aged care service provider & NSW & No \\
\hline NDIA & Statutory agency & $\mathrm{VIC}$ & No \\
\hline NDIA & Statutory agency & NSW & No \\
\hline New Horizons & Disability service provider & NSW & No \\
\hline Northcott & Disability service and housing provider & NSW & Yes (Online Focus Group 1) \\
\hline Novita Tech & Disability Service Provider & NSW & No \\
\hline Novita & Disability Service Provider & SA & Yes (SA Focus Group) \\
\hline Oak Possability & Disability service and housing provider & Tasmania & No \\
\hline Paraquad & Disability service provider & NSW & No \\
\hline Paraquad Association & Disability service provider & SA & No \\
\hline PDCN & Disability service provider & NSW & No \\
\hline $\begin{array}{l}\text { Person with a disability and his own NDIS } \\
\text { provider }\end{array}$ & End-user & SA & Yes (SA Focus Group) \\
\hline Physical Disability Council of NSW & Peak body & NSW & No \\
\hline Property Council & Statutory agency & NSW & No \\
\hline Real Estate Institute & Peak Body & NSW & No \\
\hline Rossdale Homes & Housing developer & SA & Yes (SA Focus Group) \\
\hline Scope Home Access & Aged and disability service provider & NSW & Yes (Online Focus Group 2) \\
\hline Southern Cross Care & Aged care and housing provider & Tasmania & No \\
\hline Spine Care & Disability service provider & NSW & No \\
\hline St Giles & Disability service provider & Tasmania & Yes (Online Focus Group 1) \\
\hline St John of God & Disability service and housing provider & WA & Yes (Online Focus Group 1) \\
\hline Stockland Cardinal Feeman & Aged care and housing provider & NSW & No \\
\hline Summer Housing & Disability housing provider & NSW & No \\
\hline
\end{tabular}


Appendix 4: List of invited focus

group participants

\begin{tabular}{llll}
\hline Organisation Name & Type & State & Attended \\
\hline Technical Solutions & AT provider & NSW & No \\
\hline Technology for Ageing and Disability SA & AT provider & SA & No \\
\hline Uniting communities & $\begin{array}{l}\text { Aged and disability service and } \\
\text { housing provider }\end{array}$ & SA & No \\
\hline Total number of invited participants & & 64 \\
\hline Total number of participants across all focus groups & & 24 \\
\hline
\end{tabular}




\section{Appendix 5: Focus group summaries}

\section{NSW focus group one}

Wednesday 9 October 2019, 3.00 to 4.00 pm

Held at the University of New South Wales

This focus group was one of five undertaken as part of a study to understand the implications of new and emerging AT for older people and those living with a disability from the perspective of stakeholders across disability, aged care organisations and housing providers. Of the five focus groups, three were held face-to-face in New South Wales (NSW) [2] and South Australia [1], and two online.

Thirty people representing a number of organisations (covering disability, housing providers, builders, aged care providers, peak organisations) were invited to attend the focus group in Sydney. Invitees were provided with two focus group dates. For the first focus group in NSW, three participants attended (see Table 14 for further details).

The focus group ran for just over one hour and the session was recorded and transcribed. Attendees were first asked to introduce themselves and their organisation and to give an overview of their knowledge, experience and interest in smart home AT to provide context for the discussion. The people who attended were:

- business transformation and technology executive from a disability services provider who manages the property and technology engagements of the company; including making investment decisions on AT for the accommodation stock, overseeing implementation process' of these technologies and assessment of the success of technologies for the business and clients

- a data analysist from a community housing provider who is interested in the current smart home technologies that will provide better accommodation solutions for people living with physical and mental disability

- head of strategy and innovation for aged care provider who has had several experiments and attempts in implementing various smart home technologies within their accommodation developments with the aim to improve quality-of-life for ageing Australians.

Following this, discussions focused around three key questions:

1. What do you see as the pros and cons of smart home AT in supporting independent living, quality-of-life and care provision?

2. In your opinion are there any potential risks (perceived or actual) with the management of data privacy. ie. personal day to day information about vulnerable people being shared with NGOs and private companies and potential exposure to hackers?

3. What changes would you like to see for smart home AT to be more widely deployed and adopted? 
Smart home technology was seen by the focus group participants as a means to enabling people to remain at home for longer, and for people with disability in particular to have independence from carers all the time. Care and housing providers were particularly positive about the impacts of this type of technology for their clients. However, challenges arise in selection and implementation process. Participants emphasised that the lack of best practice frameworks and guidelines make it difficult to select the most appropriate technologies for both the organisation and the client. In addition, the unaccounted costs and investment required to have the technologies functioning as they should is heavily reliant on staff knowledge and training.

Data privacy was seen as an issue and of concern to the participants in the focus group as loT technology has inherently low levels of security, creating high risks for sensitive information on vulnerable groups to be hacked. Participants in the focus group highlighted the risk of information being leaked during the transfer process between organisation and organisation. Developing data privacy management, consent and cyber security protocols and procedures is seen as a critical aspect in ensuring smart home technologies can be implemented safely and appropriately.

In terms of changes needed for smart home AT to be more widely deployed and adopted, the points raised were:

- guidelines need to be developed for best practice frameworks

- assessments for smart home technologies as assistive technologies would be extremely sought after to make informed decisions

- a need for NDIS to recognise the unaccounted costs beyond the technology itself.

Table A1: New South Wales focus group one attendees

\begin{tabular}{llrr}
\hline Organisation name & Organisation type & State & Attendees \\
\hline Achieve Australia & Housing Provider & NSW & 1 \\
\hline Hume Housing & Housing Provider & NSW & 1 \\
\hline IRT Group & Aged care and housing provider & NSW & 1 \\
\hline Total Number of Attendees & & 3
\end{tabular}

\section{NSW focus group two}

Wednesday 29 October 2019, 1.00 to 2.00 pm

Held at the University of New South Wales

This focus group was one of five undertaken as part of a study to understand the implications of new and emerging Assistive Technologies (AT) for older people and those living with a disability from the perspective of stakeholders across disability, aged care organisations and housing providers. Of the five focus groups, three were held face-toface in NSW [2] and SA [1] and two online.

Thirty people representing several organisations covering (disability, housing providers, builders, aged care providers, peak organisations), were invited to attend the focus group in Sydney. Invitees were provided with two focus group dates. For the second focus group in NSW, five participants attended (see Table A1 for further details).

The focus group ran for just over one hour and the session was recorded and transcribed. Attendees were first asked to introduce themselves and their organisation and to give an overview of their knowledge, experience and interest in smart home assistive technologies to provide context for the discussion. The people who attended were:

- executive officer for an organisation that provides home modifiers and devices that fall under the AT category who is interested in research of new and emerging technologies

- senior policy officer from a peak organisation that provides advice and guidelines on AT for ageing Australians 
- manager of service improvement innovation in a community housing provider who is interested in incorporating new and emerging AT to support ageing in place

- procurement and property services manager from a housing and aged care organisation who is interested in understanding the current developments in new and emerging AT

- project manager at a support and service provider to people on various disability schemes with a primary focus on severe disabilities.

Following this, discussions focused around three key questions:

1. What do you see as the pros and cons of smart home assistive technologies in supporting independent living, quality-of-life and care provision?

2. In your opinion are there any potential risks (perceived or actual) with the management of data privacy ie. personal day to day information about vulnerable people being shared with NGOs and private companies and potential exposure to hackers?

3. What changes would you like to see for smart home assistive technologies to be more widely deployed and adopted?

Smart home technology was seen by the focus group participants as a means to enabling people to remain at home for longer, and for people with disability in particular to have independence from carers all the time. Care and housing providers were positive about the impacts of this type of technology for their clients, particularly for those with severe spinal cord injuries. Multiple organisations have implemented voice-controlled technologies in their housing facilities and want to implement more.

Equity issues were raised, with a focus on the fact that the people who will benefit from these life changing technologies will be heavily dependent on income, status and education. Social housing providers emphasised their inability to provide such technologies as it involves home modifications for the product to function as it should. Social housing and community housing providers are, by default, excluded from home modification funding from the aged care system and NDIS. This presents a big challenge where AT is no longer stand-alone products and require integration into the home.

Data privacy was a big issue of concern to the focus group participants as loT technology has inherently low levels of security, creating high risks for sensitive information on vulnerable groups to be hacked. Participants are interested in collecting data to understand how effective the technologies are, whilst at the same time facing challenges with ethical issues of privacy and lack of coherent security systems for information transfer. Data governance and data strategy is seen as a critical component for organisations to develop. The consent process and educating clients on privacy issues that are inherent with the technology is also important, such as information being transferred back to internet companies such as Google or Amazon. The ethical issues of exploitation and discrimination was also raised when data is misused, such as sensitive information being used to determine individualised insurance schemes.

In terms of changes needed for smart home AT to be more widely deployed and adopted, the points raised were:

- building capacity through better educated clinicians and healthcare providers, particularly for universities to include new and emerging AT in their curriculum

- frameworks for assessment of technologies

- consistency in the assessment process in the NDIS scheme across states

- promotion and awareness campaigns about the technology. Educating end-users about the benefits, data privacy and procurement processes for an increasingly client direct care model. 
Table A2: New South Wales focus group two attendees

\begin{tabular}{llrr}
\hline Organisation name & Organisation type & State & Attendees \\
\hline Assistive Technologies Australia & Peak Body & NSW & 1 \\
\hline CHIA & Housing provider & NSW & 1 \\
\hline COTA NSW & Statutory agency & NSW & 1 \\
\hline Fresh Hope Care & Aged care and housing provider & NSW & 1 \\
\hline iCare & Insurance and care provider & NSW & 1 \\
\hline Total Number of Attendees & & 5 \\
\hline
\end{tabular}

\section{SOUTH AUSTRALIA focus group}

Thursday 24 October 2019, 9.30am-10.30am

Held at The University of Adelaide

This focus group was one of five undertaken as part of a study to understand the implications of new and emerging Assistive Technologies (AT) for older people and those living with a disability from the perspective of stakeholders across disability, aged care organisations and housing providers. Of the five focus groups, three were held face-toface in NSW [2] and SA [1] and two online.

Sixteen people representing several organisations covering (disability, housing providers, builders, aged care providers, peak organisations) and a person with a disability, were invited to attend the focus group in Adelaide. In all six participants attended (see for Table A2 further details).

The focus group ran for just over one hour and the session was recorded and transcribed. Attendees were first asked to introduce themselves and their organisation and to give an overview of their knowledge, experience and interest in smart home assistive technologies to provide context for the discussion. The people who attended were:

- an occupational therapist with the AT service at a disability organisation who is involved in the assessment, prescription, set-up and training phases and ongoing assistance

- a co-design manager at an aged care and housing provider currently involved in a smart home project looking at the organisations housing and how smart type solutions for older people can be implemented

- executive manager, customer stakeholder engagement at a disability services provider with an interest in the use of technology (automation and voice control) for their customers with complex and multiple disability

- person from digital transformation office of aged care and housing provider. Organisation is interested in exploring technology like loT to monitor client's lives and activities in the home

- manager of home life division of a commercial building company

- person with a disability and acting as their own NDIS provider.

Following this, discussions focused around three key questions:

1. What do you see as the pros and cons of smart home assistive technologies in supporting independent living, quality-of-life and care provision?

2. In your opinion are there any potential risks (perceived or actual) with the management of data privacy i.e. personal day to day information about vulnerable people being shared with NGOs and private companies and potential exposure to hackers?

3. What changes would you like to see for smart home assistive technologies to be more widely deployed and adopted? 
Smart home technology was seen by the focus group participants as a means to enabling people to remain at home for longer, and for people with disability in particular to have independence from carers all the time. It was noted that older people can be quite suspicious and unaccepting of this type of technology. Although, they are accepting of monitored alarms and smart technology was seen as an extension of this technology. Essentially it was felt that people will make trade-offs: use of technology that lead to improvements in their life against the scepticism and concern about privacy issues.

Some issues were raised about who is responsible for the maintenance of smart technology and who can help with issues. When a home is hard wired, the responsibility sits with the builder or installer but with smart technology there was concern this responsibility could fall onto carers - should they be expected to understand how different systems work and have to play a role in 'working' each system in different people's homes. Redundancy of the infrastructure was a considerable discussion point in the focus group. It was raised in the focus group that in the process of planning and obtaining approval for the building of disability housing the AT infrastructure had changed considerably and this presented issues.

Data privacy was an issue and of concern to the participants in the focus group but there was also a feeling that privacy has been compromised so much already that we are becoming more complacent about it. The participants believed that every family would balance the pros and cons of privacy issues and make judgments that were in the best interests of the person. They felt that in many cases if the technology (and or surveillance) provided greater 'freedom' and 'independence' then compromising data privacy was worth gaining more personal privacy from carers and service providers particularly if family could monitor systems.

In terms of changes needed for smart home AT to be more widely deployed and adopted the points raised were:

- the need for greater education about what technology in the home could look like

- better planning in terms of what is on the horizon in technological developments

- dealing with the mismatch between what can be funded and provided, and at what level, through NDIS and Aged Care Packages in terms of AT

- better integration of a range of systems - can they talk to each other?

Table A3: South Australia focus group attendees

\begin{tabular}{lllr}
\hline Organisation Name & Organisation Type & State & Attendees \\
\hline ACH & Aged care and housing provider & SA & 1 \\
\hline CARA & Disability service provider & SA & 1 \\
\hline ECH & Aged care and housing provider & SA & 1 \\
\hline Rossdale Homes & Housing Provider & SA & 1 \\
\hline Person with a disability and his own NDIS provider & End-user & SA & 1 \\
\hline NOVITA & Disability service provider & SA & 1 \\
\hline Total Number of Attendees & & 6
\end{tabular}

\section{Online Zoom focus group one}

Monday 21 October 2019, 2.30 to 3.30 pm 
This focus group was one of five undertaken as part of a study to understand the implications of new and emerging Assistive Technologies (AT) for older people and those living with a disability from the perspective of stakeholders across disability, aged care organisations and housing providers. Of the five focus groups, three were held face-toface in NSW [2] and SA [1] and two online. The online focus groups were established to facilitate participation from stakeholders across multiple locations in Tasmania, as well as participation from stakeholders in other states and/ or those who were unable to attend the face-to-face sessions.

There were six attendees at the first online focus group comprising four from Tasmania, one from Western Australia (WA) and one from NSW. Two attendees were representatives of organisations that provide both housing and other supports for the aged care and disability sectors, with the remaining four being representatives from disability and aged care organisations who provide advice and support on AT for independent living (see Table A3).

The focus group ran for one hour and was conducted via Zoom. Participants were able to contribute both by speaking and by using the chat function. The session was recorded and transcribed.

Attendees were first asked to introduce themselves and their organisation and to give an overview of their knowledge, experience and interest in smart home assistive technologies to provide context for the discussion. Following this, discussions focused around three key questions:

1. What do you see as the pros and cons of smart home assistive technologies in supporting independent living, quality-of-life and care provision?

2. In your opinion are there any potential risks (perceived or actual) with the management of data privacy i.e. personal day to day information about vulnerable people being shared with NGOs and private companies and potential exposure to hackers?

3. What changes would you like to see for smart home assistive technologies to be more widely deployed and adopted?

In relation to the pros and cons of smart home AT in supporting independent living, quality-of-life and care provision, generally attendees acknowledged the value and benefits of AT for their clients, including improvements in qualityof-life and supporting their capacity to live independently. Participants noted that technologies could be tailored to the unique needs and circumstances of their clients. Even within the aged care sector, one participant noted the 'surprisingly' good uptake and benefits to residents in reducing feelings of social isolation. However, some participants expressed concern at the risk of AT replacing person-to-person supports. Other challenges discussed included knowledge transfer and levels of technological literacy for both care support workers and clients, leading to the risk of AT not being utilised effectively.

The availability of smart technologies at relatively low upfront cost was seen to be a benefit; however, participants also noted the limitations of 'off-the-shelf' products to meet the needs of their clients. While the availability of appropriate technologies was improving, participants stated that there were some limitations in accessing technologies available overseas. The need to modify AT and to integrate discrete technologies as part of a smart home AT system were seen to present the greatest challenges, particularly in relation to funding. Access to funding for the integration of AT and for ongoing versus upfront costs was seen to be less certain and funding for the purchase of AT was generally seen to be inconsistent. The rapidity of development in AT was also seen to present a challenge, both in keeping abreast of the availability of new technologies and the risk of redundancy within a short time period.

Participants agreed that many technologies could be readily integrated in housing, especially in new-builds with the industry shifting towards smart homes as common practice. The ability to consider the design of the technology and the design of the dwelling as part of a whole system was seen to be particularly beneficial. However, participants noted some difficulties in interacting with housing authorities and the challenges of integrating AT in the private rental market. Larger houses and group home settings also presented some technological challenges. There was also a recognised need for smart design in the integration of AT with built-in flexibility for longevity to suit a range of potential future residents with different needs. 
Data privacy was seen to be a key area of concern. Identified risks included holding data in cloud-based infrastructure, tier-to-tier transmission, lack of clarity around data ownership, difficulties in comprehensively communicating data privacy issues to clients, and difficulties with clients remembering passwords leading to organisations maintaining the capability of full access to personal information. Developing more robust policies, procedures and standards for managing data privacy was seen to be a key need.

Another key area of development identified to support the deployment and adoption of AT was increasing access to funding. This included broadening the scope of the definition of AT in relation to 'reasonable and necessary' supports, access to funding to support modifications and systems integration of AT as well as capital costs, considering funding holistically in relation to ongoing benefits versus upfront costs, and ensuring access to skilled professionals to prescribe and recommend appropriate AT, particularly in regional areas. Additionally, there was an identified need to address the risk of redundancy of AT infrastructure through guarantees in Australian consumer law.

Table A4: Online Zoom focus group one attendees

\begin{tabular}{lllr}
\hline Organisation name & Organisation type & \multicolumn{2}{l}{ State } \\
\hline Anglicare & Aged and disability service and housing provider & Tasmania & 1 \\
\hline Community Based Support & Disability service and housing provider & Tasmania & 1 \\
\hline Independent Living Centre & Peak body & Tasmania & 1 \\
\hline Northcott & Disability service and housing provider & NSW & 1 \\
\hline St Giles & Disability service provider & Tasmania & 1 \\
\hline St John of God & Disability accommodation and support & WA & 1 \\
\hline Total Number of Attendees & & & 6 \\
\hline
\end{tabular}

\section{Online Zoom focus group two}

Monday 21 October 2019, 4.00 to 5.00 pm

This focus group was one of five undertaken as part of a study to understand the implications of new and emerging Assistive Technologies (AT) for older people and those living with a disability from the perspective of stakeholders across disability, aged care organisations and housing providers. Of the five focus groups, three were held face-toface in NSW [2] and SA [1] and two online. The online focus groups were established to facilitate participation from stakeholders across multiple locations in Tasmania, as well as participation from stakeholders in other states and/ or those who were unable to attend the face-to-face sessions.

There were five attendees at the second online focus group comprising two from Tasmania, one from Victoria and two from NSW. One attendee was a representative from a disability housing and care provider, two were representatives from organisations who provide advice and support on Assistive Technology for independent living, one was a representative from a provider of AT and one was a representative from the NDIS (see Table A4). Due to technology difficulties, the representative from the NDIA was unable to fully participate in the meeting.

The focus group ran for one hour and was conducted via zoom. Participants were able to contribute both by speaking and by using the chat function. The session was recorded and transcribed. Attendees were first asked to introduce themselves and their organisation and to give an overview of their knowledge, experience and interest in smart home assistive technologies to provide context for the discussion. Following this, discussions focused around three key questions:

1. What do you see as the pros and cons of smart home assistive technologies in supporting independent living, quality-of-life and care provision? 
2. In your opinion are there any potential risks (perceived or actual) with the management of data privacy i.e. personal day to day information about vulnerable people being shared with NGOs and private companies and potential exposure to hackers?

3. What changes would you like to see for smart home assistive technologies to be more widely deployed and adopted?

In relation to the pros and cons of smart home AT in supporting independent living, quality-of-life and care provision, generally attendees acknowledged the value and benefits of AT for their clients including fostering greater autonomy, safety and supporting their capacity to live independently. However, there was also discussion about differing perceptions of the benefits and value of AT and its integration with person-to-person care between families and end-users. Participants also discussed the potential of AT to support proactive rather than reactive care through data-driven decision-making but noted that this would require transformations in care delivery models.

Technological developments and the increasing range and availability of smart technologies at 'right size and right price' was seen to be a key opportunity in increasing uptake of AT. However, this was also seen to present a barrier in the ability of end-users, carers, assessors and organisations to keep abreast of information and make informed decisions leading to a 'reluctance to go into this space'. Participants discussed challenges of creating integrated systems that were robust and durable, including the limitations of longevity with cloud-based services and the risks where systems were operated by multiple users. Rural areas were identified as presenting challenges in relation to internet reliability and access to support services. While the availability of appropriate technologies was improving, participants stated that there were some limitations in accessing technologies or components from overseas.

Participants agreed that emerging smart technologies more readily facilitated retrofitting in existing residences, including rental accommodation. However, the diversity of needs and housing circumstances could present challenges leading to the need for complex and situation specific modifications.

Data privacy was seen to be a key area of concern including the 'suspicion factor' and perceptions of potential privacy breaches with the use of cameras and Artificial Intelligence. Participants noted the 'fine balance' that needs to be maintained between privacy and autonomy. Developing more robust policies, procedures and standards for managing data privacy was seen to be a key need.

Another area of development identified to support the deployment and adoption of AT was increasing access to funding. First, this included funding to support professional development and training. Second, there is a need for more detailed assessments and for more tailored AT solutions. Third there was an increased level of support required for rural and regional areas, including access to skilled professionals to prescribe and recommend appropriate AT. Greater support and security to allow businesses to innovate and invest in this space was also noted as a need, as well as policies and standards to address the risk of redundancy of AT infrastructure.

Table A5: Online Zoom focus group two attendees

\begin{tabular}{lllr}
\hline Organisation name & Organisation type & State \\
\hline Homestay & Housing provider & NSW & 1 \\
\hline Li-Ve & Disability service and housing provider & Tasmania & 1 \\
\hline Independent Living Centre & Peak body & Tasmania & 1 \\
\hline Scope Home Access & Aged and disability service and housing provider & NSW & 1 \\
\hline Total Number of Attendees & & 4 \\
\hline
\end{tabular}


Table A6: Summary list of all attendees at all focus groups

\begin{tabular}{|c|c|c|c|}
\hline Organisation Name & Organisation Type & State & Attendees \\
\hline \multicolumn{4}{|l|}{ New South Wales Focus Group 1} \\
\hline Achieve Australia & Housing Provider & NSW & 1 \\
\hline Hume Housing & Housing Provider & NSW & 1 \\
\hline IRT Group & Aged care and housing provider & NSW & 1 \\
\hline Total Number of Attendees & & & 3 \\
\hline \multicolumn{4}{|l|}{ New South Wales Focus Group 2} \\
\hline Assistive Technologies Australia & AT Provider & NSW & 1 \\
\hline $\mathrm{CHIA}$ & Housing provider & NSW & 1 \\
\hline COTA NSW & Statutory agency & NSW & 1 \\
\hline Fresh Hope Care & Aged care and housing provider & NSW & 1 \\
\hline iCare & Insurance and care provider & NSW & 1 \\
\hline Total Number of Attendees & & & 5 \\
\hline \multicolumn{4}{|l|}{ South Australia } \\
\hline $\mathrm{ACH}$ & Aged care and housing provider & SA & 1 \\
\hline CARA & Disability service provider & SA & 1 \\
\hline $\mathrm{ECH}$ & Aged care and housing provider & SA & 1 \\
\hline Rossdale Homes & Housing Provider & SA & 1 \\
\hline Person with a disability and his own NDIS provider & End-user & SA & 1 \\
\hline NOVITA & Disability service provider & SA & 1 \\
\hline Total Number of Attendees & & & 6 \\
\hline \multicolumn{4}{|l|}{ Online Zoom Focus Group 1} \\
\hline Anglicare & Aged and disability service and housing provider & Tasmania & 1 \\
\hline Community Based Support & Disability service and housing provider & Tasmania & 1 \\
\hline Independent Living Centre & Peak body & Tasmania & 1 \\
\hline Northcott & Disability service and housing provider & NSW & 1 \\
\hline St Giles & Disability service provider & Tasmania & 1 \\
\hline St John of God & Disability accommodation and support & WA & 1 \\
\hline Total Number of Attendees & & & 6 \\
\hline \multicolumn{4}{|l|}{ Online Zoom Focus Group 2} \\
\hline Homestay & Housing provider & NSW & 1 \\
\hline Li-Ve & Disability service and housing provider & Tasmania & 1 \\
\hline Independent Living Centre & Peak body & Tasmania & 1 \\
\hline Scope Home Access & Aged and disability service and housing provider & NSW & 1 \\
\hline Total Number of Attendees & & & 4 \\
\hline
\end{tabular}




\section{Appendix 6: NDIS SDA guidelines mention of automation or smart homes}

Table A7: NDIS SDA guidelines appendix A mention of automation or smart homes

\begin{tabular}{|c|c|c|c|}
\hline Clause & Design requirements & Rationale & Applicable to \\
\hline \multicolumn{4}{|c|}{ 2. Pedestrian entry from the site boundary } \\
\hline 2.11 & $\begin{array}{l}\text { Where a ramp or walkway is part of the } \\
\text { accessway, level landings no less than } \\
1200 \mathrm{~mm} \times 1200 \mathrm{~mm} \text {, exclusive to the swing of } \\
\text { the door or gate that opens onto them, shall } \\
\text { be provided. The landing size shall increase } \\
\text { based on the required gate / door circulation } \\
\text { spaces or change of direction requirements as } \\
\text { per AS1428.1. }\end{array}$ & $\begin{array}{l}\text { Door circulation requirements in } \\
\text { accordance with AS1428.1 are essential for } \\
\text { a person using a wheelchair to be able to } \\
\text { operate the door or gate independent of a } \\
\text { carer. } \\
\text { Door automation at the very onset is a } \\
\text { suitable solution in lieu of the provision of } \\
\text { latch side door circulation spaces. }\end{array}$ & $\begin{array}{l}\text { - Fully Accessible } \\
\text { - High Physical } \\
\text { Support }\end{array}$ \\
\hline \multicolumn{4}{|c|}{ 4. Entrance doorway and door hardware } \\
\hline 4. 1.3 & $\begin{array}{l}\text { All door circulation spaces (excluding internal } \\
\text { door circulation spaces for bedrooms) shall be } \\
\text { provided in accordance with AS } 1428.1 \text { to both } \\
\text { sides of the door. }\end{array}$ & $\begin{array}{l}\text { Compliance with AS1428.1 is considered to } \\
\text { be relevant as it sets out the requirements } \\
\text { generally for the } 80 \text { th percentile wheelchair } \\
\text { size except at accessways at the location } \\
\text { of a turn greater than } 60^{\circ} \text {, sanitary facilities } \\
\text { and at doorways, including door width and } \\
\text { circulation space, where it provides suitable } \\
\text { sizes for the } 90 \text { th percentile wheelchair size. } \\
\text { Note that doors which swing outside, } \\
\text { including any screen doors will also be } \\
\text { required to provide door circulation spaces } \\
\text { as per AS1428.1 } \\
\text { A concession to this requirement can } \\
\text { be applied to storage and exclusive staff } \\
\text { use areas where staff accommodation is } \\
\text { provided as a part of the dwelling. } \\
\text { Note that door automation cannot replace } \\
\text { door circulation space length however door } \\
\text { automation is considered an appropriate } \\
\text { BCA performance solution in lieu of door } \\
\text { latch side clearance. } \\
\text { For internal door circulation spaces for } \\
\text { bedrooms refer to Figure } 16 \text {. }\end{array}$ & $\begin{array}{l}\text { - Fully Accessible } \\
\text { - High Physical } \\
\text { Support }\end{array}$ \\
\hline
\end{tabular}




\begin{tabular}{|c|c|c|c|}
\hline Clause & Design requirements & Rationale & Applicable to \\
\hline \multirow[t]{4}{*}{ 4. 2.7} & \multirow{2}{*}{$\begin{array}{l}\text { Doorway circulation spaces in accordance } \\
\text { with AS1428.1 shall be provided to all doorways } \\
\text { (excluding exclusive staff use areas, non- } \\
\text { accessible toilets and storage rooms). }\end{array}$} & To enable safe and easy access to doors. & - Fully Accessible \\
\hline & & \multirow{3}{*}{$\begin{array}{l}\text { Note that Door automation cannot replace } \\
\text { door circulation space length however door } \\
\text { automation is considered an appropriate } \\
\text { BCA performance solution in lieu of door } \\
\text { latch side clearance. }\end{array}$} & $\begin{array}{l}\text { - High Physical } \\
\text { Support }\end{array}$ \\
\hline & $\begin{array}{l}\text { Door automation can be provided in lieu door } \\
\text { circulation spaces that are required as per } \\
\text { AS1428.1 to the latch side of the door. }\end{array}$ & & \\
\hline & $\begin{array}{l}\text { Note that if door circulation is not provided as } \\
\text { per AS1428.1 then door automation must be } \\
\text { provided at the onset and not simply provision } \\
\text { for door automation at a future time. }\end{array}$ & & \\
\hline \multirow[t]{2}{*}{ 4. 2.8} & $\begin{array}{l}\text { Power and control cabling to head of entry } \\
\text { doors of participant bedrooms, one external } \\
\text { entry doorway and one external doorway to an } \\
\text { open area (where provided) shall be provided } \\
\text { for future automation. }\end{array}$ & & $\begin{array}{l}\text { - High Physical } \\
\text { Support }\end{array}$ \\
\hline & $\begin{array}{l}\text { A capped GPO at the door head may be } \\
\text { provided to comply with this requirement. }\end{array}$ & & \\
\hline \multicolumn{4}{|c|}{ 6. Windows } \\
\hline 6.3 & $\begin{array}{l}\text { Power and control cabling to windows of } \\
\text { bedrooms and living areas shall be provided } \\
\text { for future window blind automation. }\end{array}$ & \multirow{2}{*}{$\begin{array}{l}\text { Provided to enable operation of window } \\
\text { covering, where manual operation is difficult } \\
\text { through future automation. Automation in } \\
\text { the future will allow opening and closing of } \\
\text { window furnishing automatically by a remote } \\
\text { control or a push button. }\end{array}$} & $\begin{array}{l}\text { - High Physical } \\
\text { Support }\end{array}$ \\
\hline & $\begin{array}{l}\text { A capped GPO at the window head may be } \\
\text { provided to comply with this requirement. }\end{array}$ & & \\
\hline \multicolumn{4}{|c|}{ Appendix A: General best practice recommendations } \\
\hline \multirow[t]{2}{*}{7} & Sanitary facilities & \multicolumn{2}{|l|}{ For all design categories: } \\
\hline & & \multicolumn{2}{|c|}{$\begin{array}{l}\text { - It is recommended to provide for power supply for future sensor } \\
\text { operations of taps, toilet and lighting in sanitary facilities. }\end{array}$} \\
\hline \multirow[t]{2}{*}{8} & Kitchen & \multicolumn{2}{|l|}{ For all design categories: } \\
\hline & & \multicolumn{2}{|c|}{$\begin{array}{l}\text { - It is recommended to provide automation of height-adjustable } \\
\text { benchtop along with a safety mechanism and large buttons in } \\
\text { front of benchtop edge to enable automation. }\end{array}$} \\
\hline 21 & Heating and cooling & \multicolumn{2}{|c|}{$\begin{array}{l}\text { - It is recommended to provide technology such as controls of } \\
\text { air-conditioning to be operable via smart devices like iPads etc. }\end{array}$} \\
\hline 24 & Assistive Technology & \multicolumn{2}{|c|}{$\begin{array}{l}\text { - It is recommended to provide video or intercom systems for the } \\
\text { Fully Accessible design category. }\end{array}$} \\
\hline 25 & Fire safe design & \multicolumn{2}{|c|}{$\begin{array}{l}\text { - It is recommended to provide automatic sprinkler system for all 2+ } \\
\text { participant dwellings. This may be offset by fire compartmentation } \\
\text { in some buildings that are not sprinkler protected and may require } \\
\text { fire engineering. }\end{array}$} \\
\hline
\end{tabular}

Source: (NDIS 2019b). 


\section{AHURi}

Australian Housing and Urban Research Institute Level 12, 460 Bourke Street

Melbourne VIC 3000

\section{Australia}

+61396602300

information@ahuri.edu.au

ahuri.edu.au

(2) twitter.com/AHURI_Research

f facebook.com/AHURI.AUS

in Australian Housing and Urban Research Institute 